\title{
DORIVAL JULIO DELLA TOGNA
}

Efeito da correção cirúrgica nas variáveis do teste cardiopulmonar de exercício em pacientes com insuficiência mitral crônica

Tese apresentada ao Instituto Dante Pazzanese de Cardiologia, Entidade Associada da Universidade de São Paulo para a obtenção do título de Doutor em Ciências.

\section{VERSÃO CORRIGIDA}




\section{DORIVAL JULIO DELLA TOGNA}

Efeito da correção cirúrgica nas variáveis do teste cardiopulmonar de exercício em pacientes com insuficiência mitral crônica

Tese apresentada ao Instituto Dante Pazzanese de Cardiologia, Entidade Associada da Universidade de São Paulo para a obtenção do título de Doutor em Ciências.

Área de concentração: Medicina, Tecnologia e Intervenção em Cardiologia

Orientador: Prof. Dr. Alexandre Antonio Cunha Abizaid 
Dados Internacionais de Catalogação na Publicação (CIP) Preparada pela Biblioteca do Instituto Dante Pazzanese de Cardiologia Oreprodução autorizada pelo autor

Togna, Dorival Julio Della

Efeito da correção cirúrgica nas variáveis do teste cardiopulmonar de exercício em pacientes com insuficiência mitral crônica/ Dorival Julio Della Togna. — São Paulo, 2012.

Tese(doutorado)--Instituto Dante Pazzanese de Cardiologia, Universidade de São Paulo.

Área de Concentração: Medicina, Tecnologia e Intervenção em Cardiologia

Orientador: Alexandre Antônio da Cunha Abizaid

Descritores: 1.INSUFICIÊNCIA DA VALVA MITRAL. 2.SINTOMAS. 3.EXERCÍCIO. 4. CIRURGIA CARDÍACA

USP/IDPC/Biblioteca-016/12 
Dedicatória 
Aos meus pais, Dorival (in memorian) e Neuza ............

À minha esposa Ana Lúcia e aos meus filhos André e Tiago.............

Aos pacientes que carinhosamente aceitaram participar deste estudo.............

À Deus................................. 
"O amor é algo eterno, o aspecto pode mudar, mas não a essência."

\section{Vincent Willem van Gogh}

"Quando a gente acha que tem todas as respostas, vem a vida e muda todas as perguntas."

Luís Fernando Verissimo

"Sabemos de quase nada adequadamente, de poucas coisas a priori, e da maioria por meio da experiência." 
"Há verdadeiramente duas coisas diferentes:

saber e crer que se sabe. A ciência consiste em

saber; em crer que se sabe reside a ignorância."

Hipócrates 
Agradecimentos 
Ao meu orientador Dr. Alexandre Abizaid pelo conhecimento, precisos aconselhamentos, sabedoria e compreensão

Ao Dr. Romeu Meneghelo pela valiosa contribuição na análise e interpretação do teste cardiopulmonar de exercício

À Dra. Zilda Meneghelo pela oportunidade de aprendizado diário, sendo exemplo de conduta ética, sabedoria e bondade

À Dra. Auristela pela amizade, aprendizado e acolhimento no árduo e recompensador trabalho diário.

Ao Dr. David pela amizade e qualidade na realização do estudo ecocardiográfico

Aos Dr. Almir e Dr. Mastrocolla pela elaboração dos laudos do teste cardiopulmonar de exercício

À Roberta, responsável pelo difícil e fundamental trabalho de estatística

Aos colegas e amigos do Setor de Valvopatias do Instituto Dante Pazzanese de Cardiologia, Dra. Lúcia, Dra. Nísia, Dra. Idelzuíta, Dra. Cecília, Dra. Samira, Dr. Eduardo, Dra.Rosa e Dr. Carlos, pelo apoio, incentivo, companheirismo e agradável convívio diário 
Aos médicos residentes do Setor de Hemodinâmica, Dr. Felipe, Dr. Carlos Collet e Dr. Alejandro pelo valioso apoio na realização do cateterismo cardíaco

Aos amigos e funcionários do setor de Valvopatias, em especial ao Sr. Paulo Nascimento, fundamental na organização, apoio logístico e cumprimento do cronograma

Aos funcionários dos setores de Reabilitação, Hemodinâmica e Ecocardiografia do Instituto Dante Pazzanese de Cardiologia, sem os quais não seria possível a realização deste estudo

Aos amigos e familiares, em especial às minhas irmãs Gisele e Carla 


\section{SUMÁRIO}

Lista de Siglas e Abreviações

Lista de Figuras

Lista de Tabelas

Resumo

Summary

1 INTRODUÇÃO-- 01

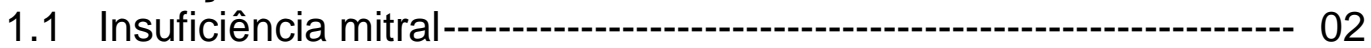

1.2 Etiologia da insuficiência mitral -----on 03

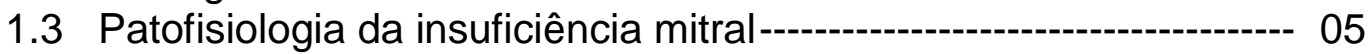

1.4 Manejo clínico e indicação cirúrgica na insuficiência mitral -------- 08

1.5 Avaliação da Classe Funcional na insuficiência mitral ------------- 11

2 OBJETIVOS ----_os 19

3 MÉTODOS - 21

3.1 Seleção de pacientes-- 22

3.1.1 Critérios de inclusão ------------------------------------- 22

3.1.2 Critérios de exclusão -----.-- 23

3.2 Desenho do estudo --- 24

3.3 Tamanho amostral ---.-- 24

3.4 Protocolo de avaliação dos pacientes ---------- 25

3.4.1 Anamnese, exame físico, indicação cirúrgica e etiologia da insuficiência mitral---_-_--- 25

3.4.2 Eletrocardiograma-----ond-- 26

3.4.3 Exames laboratoriais sanguíneos---------------------------- 26

3.4.4 Ecocardiografia trans-torácica ---------------------------- 26

3.4.5 Teste cardiopulmonar de exercício --------------------------- 31

3.4.6 Cateterismo cardíaco------o--- 36

3.4.7 Método estatístico ---_- 39

4 RESULTADOS--- 42

4.1 Características da população --- 43

4.2 Cirurgia da valva mitral: escolha da prótese, alterações anatômicas do aparato mitral e técnicas de reparo ------- 45

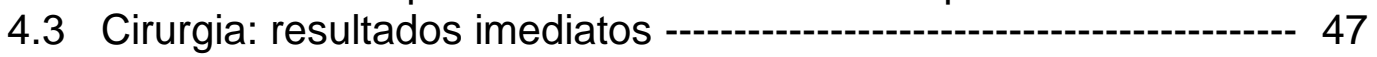

4.4 Variáveis clínicas e laboratoriais pré e pós-operatório ------------- 48

4.5 Ecocardiograma pré e pós-operatório ------------------------- 51

4.6 Estudo hemodinâmico pré-operatório e comparação com as medidas do ecocardiograma ------os 54

4.7 Prova de função pulmonar pré e pós-operatório---------------- 55

4.8 Teste cardiopulmonar de exercício pré e pós-operatórios ---------- 56

4.8.1 Efeito da cirurgia nas variáveis do teste cardiopulmonar de exercício realizado na bicicleta ---_e 57

4.8.2 Efeito da cirurgia nas variáveis do teste cardiopulmonar de exercício realizado na esteira -- 60

4.8.3 Efeito da remodelamento cardíaco após a cirurgia nas variáveis do teste cardiopulmonar de exercício: medidas 
de correlação ---

4.9 Grupos troca valvar mitral e reparo mitral ----a---o 68

4.9.1 Características de base---o 69

4.9.2 Efeito da cirurgia nos parâmetros ecocardiográficos ------------- 73

4.9.3 Efeito da cirurgia nas variáveis do teste cardiopulmonar de exercício ---------------- 75

5 DISCUSSÃO -- 85

5.1 Considerações preliminares -----on 86

5.2 Teste cardiopulmonar de exercício realizado na bicicleta---------- 92

5.3 Teste cardiopulmonar de exercício realizado na esteira------------- 99

5.4 Considerações finais - -

5.5 Limitaç̃̃es do estudo - 103

6 CONCLUSÅO --- 105

7 ANEXOS---- 108

10 REFERÊNCIAS BIBLIOGRÁFICAS--- 114 
Listas 


\section{Lista de Siglas e Abreviações (em ordem alfabética)}

ACC

$\mathrm{AE}$

$\mathrm{AHA}$

AJ

$\mathrm{AJ} / \mathrm{AE}$

Ao

AOR

ASC

ATPS

BRA

bpm

BTPS

$\mathrm{CF}$

$\mathrm{cm}^{2}$

$\mathrm{CO}_{2}$

CVF

DC

DDFVE

Delta D

Dif. a-v $\mathrm{O}_{2}$

$\mathrm{dL}$

$\mathrm{DP}$

DSFVE

ECG
American College of Cardiology

átrio esquerdo

American Heart Association

área do jato regurgitante mitral

relação área do jato regurgitante mitral / área do átrio esquerdo

aorta

área do orifício de regurgitação

área de superfície corporal

Ambient Temperature and Pressure, satured - no português: temperatura e pressão ambientais, saturada com vapor d'água

bloqueador do receptor da angiotensina AT1

batimentos por minuto

Body Temperature and Pressure, satured - no português: condições corporais, temperatura corporal, pressão ambiente, saturada com vapor d'água

classe funcional

centímetro quadrado

dióxido de carbono

capacidade vital forçada

débito cardíaco

diâmetro diastólico final do ventrículo esquerdo

fração de encurtamento

diferença artério-venosa de oxigênio

decilitro

desvio padrão

diâmetro sistólico final do ventrículo esquerdo

eletrocardiograma 


\begin{tabular}{|c|c|}
\hline ECO & ecocardiograma \\
\hline et al. & e outros \\
\hline EUA & Estados Unidos da América \\
\hline FA & fibrilação atrial \\
\hline FC & freqüência cardíaca \\
\hline FE & fração de ejeção \\
\hline IC & insuficiência cardíaca \\
\hline ICC & insuficiência cardíaca congestiva \\
\hline IECA & Inibidor de enzima conversora da angiotensina \\
\hline IM & insuficiência mitral \\
\hline IMC & índice de massa corporal \\
\hline $\mathrm{Kg}$ & quilograma \\
\hline LA & limiar anaeróbico \\
\hline LV & limiar ventilatório \\
\hline$m^{2}$ & metro quadrado \\
\hline máx & máximo / a \\
\hline MET & equivalente metabólico ou unidade metabólica basal \\
\hline $\mathrm{MHz}$ & megahertz \\
\hline $\min$ & minuto \\
\hline $\mathrm{mL}$ & mililitro \\
\hline $\mathrm{mm}$ & milímetro \\
\hline $\mathrm{mm} \mathrm{Hg}$ & milímetro de mercúrio \\
\hline $\mathrm{n}$ & número de pacientes \\
\hline NS & não significativo \\
\hline NYHA & New York Heart Association \\
\hline $\mathrm{O}_{2}$ & oxigênio \\
\hline OUES & Oxygen Uptake Efficiency Slope \\
\hline PA & pressão arterial \\
\hline PAD & pressão arterial diastólica \\
\hline PAS & pressão arterial sistólica \\
\hline $\mathrm{PETCO}_{2}$ & pressão parcial de gás carbônico no final da expiração \\
\hline $\mathrm{PETO}_{2}$ & pressão parcial de oxigênio no final da expiração \\
\hline
\end{tabular}




\begin{tabular}{|c|c|}
\hline PISA & proximal isovelocity surface area \\
\hline RER & respiratory exchange ratio - no português: razão de \\
\hline & troca gasosa, relação $\mathrm{VCO}_{2} / \mathrm{VO}_{2}$ \\
\hline rpm & rotações por minuto \\
\hline RS & ritmo sinusal \\
\hline STPD & $\begin{array}{l}\text { standard temperature pressure, dry - no português: } \\
\text { condições padrão, temperatura } 0^{\circ} \mathrm{C} \text {, pressão } 760 \mathrm{mmHg} \text {, } \\
\text { a seco }\end{array}$ \\
\hline TCPE & teste cardiopulmonar de exercício \\
\hline TE & Teste ergométrico \\
\hline $\mathrm{T} 1 / 2 \mathrm{VO}_{2}$ & $\begin{array}{l}\text { tempo para que o } \mathrm{VO} \square \text { atingisse metade do seu valor } \\
\text { máximo no esforço, na fase de recuperação }\end{array}$ \\
\hline $\mathrm{VCO}_{2}$ & volume de dióxido de carbono produzido por minuto \\
\hline VD & ventrículo direito \\
\hline $\mathrm{Vd} / \mathrm{Vt}$ & relação espaço morto ventilatório / volume corrente \\
\hline VDF & volume diastólico final do ventrículo esquerdo \\
\hline VEF1 & volume expiratório forçado no primeiro segundo \\
\hline VE & ventilação pulmonar por minuto \\
\hline $\mathrm{VE} / \mathrm{VCO}_{2}$ & equivalente ventilatório para o gás carbônico \\
\hline $\mathrm{VE} / \mathrm{VCO}_{2}$ slope & $\begin{array}{l}\text { inclinação do equivalente de ventilação de gás } \\
\text { carbônico }\end{array}$ \\
\hline $\mathrm{VE} / \mathrm{VO}_{2}$ & equivalente ventilatório para o oxigênio \\
\hline $\mathrm{VO}_{2}$ & volume de oxigênio consumido por minuto \\
\hline $\mathrm{VO}_{2}$ máx & consumo máximo de oxigênio \\
\hline $\mathrm{VO}_{2}$ pico & consumo de oxigênio no ponto máximo do esforço \\
\hline $\mathrm{VO}_{2} / \mathrm{FC}$ & pulso de oxigênio \\
\hline VR & volume regurgitante \\
\hline VP & veia pulmonar \\
\hline VC & vena contracta \\
\hline vs & "versus" \\
\hline VDFVE & volume diastólico final do ventrículo esquerdo \\
\hline
\end{tabular}


VSFVE

VVM

W volume sistólico final do ventrículo esquerdo ventilação voluntária máxima

watt (carga ou potência) 


\section{Lista de Figuras}

Figura 1 - As imagens exemplificam como foram feitas as medidas dos volumes do ventrículo esquerdo por meio da técnica ecocardiográfica de Simpson, em dois planos ortogonais

Figura 2 - Demonstração de como foram obtidas as medidas do volume sistólico do átrio esquerdo em dois planos ortogonais, por meio da técnica ecocardiográfica de Simpson

Figura 3 - As imagens demonstram a medida da vena contracta (região mais estreita do jato de insuficiência, logo abaixo da coaptação dos folhetos); este método ecocardiográfico foi utilizado para a quantificação da insuficiência valvar mitral. A imagem à direita é uma seção central ampliada da imagem à esquerda, com utilização de zoom

Figura 4 - As figuras representam a técnica ecocardiográfica de PISA (proximal isovelocity surface area), utilizada para a medida da área do orifício de regurgitação (AOR) e volume de regurgitação (VR), dados fundamentais para classificar a gravidade da insuficiência valvar mitral. À esquerda, observa-se a medida do raio da zona de convergência e à direita a medida da integral da velocidade do jato de refluxo

Figura 5 - Curva do pulso de oxigênio pré-operatório. Paciente TU, 72 anos, feminino, submetida ao reparo mitral com sucesso. Observa-se que o pulso de oxigênio passou de 3 para $5 \mathrm{~mL}$.bat. ${ }^{-1}$, no início do esforço, decrescendo gradativamente na sequência, indicando redução da performance ventricular esquerda com o esforço. As linhas descontínuas indicam o início e o fim do esforço 
Figura 6 - Curva do pulso de oxigênio pós-operatório. Paciente TU, 72 anos, feminino, submetida ao reparo mitral com sucesso. O pulso de oxigênio passou para $7 \mathrm{~mL}$.bat. ${ }^{1}$ e não mais decresceu com a sequência do exercício. As linhas descontínuas indicam o início e o fim do esforço 36

Figura 7 - Estudo hemodinâmico: medidas dos volumes diastólico e sistólico final do ventrículo esquerdo e cálculo da fração de ejeção 39

Figura 8 - Gráficos da dispersão dos valores entre diferenças relativas de medidas do ECO e variáveis do TCPE realizado na bicicleta 66

Figura 9 - Gráficos da dispersão dos valores entre diferenças relativas de medidas do ECO e variáveis do TCPE realizado na esteira 69

Figura 10 - Representação esquemática do efeito de interação entre tempo e grupos (TVM e RM) nas variáveis do TCPE realizado na bicicleta 81

Figura 11 - Representação esquemática do efeito de interação entre tempo e grupos (TVM e RM) nas variáveis do TCPE realizado na esteira- 


\section{Lista de Tabelas}

Tabela 1 - Características clínicas pré-operatórias dos 72 indivíduos portadores de IM grave e incluídos no protocolo

Tabela 2 - Cirurgia: descrição das alterações anatômicas do aparato valvar mitral nos 72 pacientes incluídos no estudo

Tabela 3 - Reparo mitral: descrição das técnicas cirúrgicas realizadas nos 47 pacientes do estudo submetidos ao reparo mitral ---- 47

Tabela 4 - Características de base: quadro comparativo pré e pós-operatório

Tabela 5 - Comparação dos parâmetros ecocardiográficos de remodelamento cardíaco no pré e pós-operatórios 53

Tabela 6 - Estudo hemodinâmico pré-operatório 54

Tabela 7 - Comparação pré-operatória entre as medidas do ecocardiograma e estudo hemodinâmico

Tabela 8 - Prova de Função Pulmonar: comparação entre as medidas pré e pós-operatórias 56

Tabela 9 - Efeito da cirurgia nas variáveis dos TCPE realizados na bicicleta

Tabela 10 - Avaliação da curva $\mathrm{VO}_{2}$ /carga e pulso de $\mathrm{O}_{2}$ durante o Esforço nos TCPE realizados na bicicleta antes e após a cirurgia

Tabela 11 - Efeito da cirurgia nas variáveis do TCPE realizados na esteira

Tabela 12 - Avaliação da curva $\mathrm{VO}_{2}$ /carga e pulso de $\mathrm{O}_{2}$ durante $\mathrm{o}$ Esforço nos TCPE realizados na esteira antes e após a cirurgia 64

Tabela 13 - Efeito do remodelamento cardíaco nas variáveis do TCPE realizados na bicicleta: correlação entre as diferenças relativas do ECO e TCPE66

Tabela 14 - Efeito do remodelamento cardíaco nas variáveis do TCPE 
realizados na esteira: correlação entre as diferenças relativas do ECO e TCPE-

Tabela 15 - Características de base em pacientes submetidos à troca valvar mitral e reparo mitral-

Tabela 16 - Comparação dos exames laboratoriais pré-operatórios em pacientes submetidos à troca valvar mitral e reparo mitral-

Tabela 17 - Comparação pré-operatória dos parâmetros ecocardiográficos em pacientes submetidos à troca valvar mitral e reparo mitral-

Tabela 18 - Cateterismo cardíaco: comparação pré-operatória entre a troca valvar mitral e o reparo mitral

Tabela 19 - Comparação ecocardiográfica das medidas de remodelação nos grupos e entre os grupos troca valvar mitral e reparo mitral-

Tabela 20 - Efeito da cirurgia nas variáveis do TCPE realizados na bicicleta em pacientes submetidos à troca valvar mitral $\mathrm{e}$ reparo mitral

Tabela 21 - Valores de p da ANOVA para avaliação do efeito de Interação entre tempo e grupo para as variáveis do TCPE na bicicleta

Tabela 22 - Efeito da cirurgia nas variáveis do TCPE realizados na esteira em pacientes submetidos à troca valvar mitral e reparo mitral

Tabela 23 - Valores de p da ANOVA para avaliação do efeito de interação entre tempo e grupo para as variáveis do TCPE na esteira 


\section{RESUMO}

Della Togna, DJ. Efeito da Correção Cirúrgica nas Variáveis do Teste Cardiopulmonar de Exercício em Pacientes com Insuficiência Mitral Orgânica [tese]. São Paulo: Instituto Dante Pazzanese de Cardiologia, Universidade de São Paulo; 2012. 130p.

Introdução: A Insuficiência Mitral (IM) crônica orgânica tem fisiopatologia complexa. Os sintomas de insuficiência cardíaca e piora da capacidade funcional, podem aparecer apenas tardiamente, quando já existe disfunção contrátil do ventrículo esquerdo (VE). O teste cardiopulmonar de exercício (TCPE) pode avaliar objetivamente a capacidade funcional e auxiliar a conduta médica nos pacientes com IM, mas, pouco se conhece sobre o efeito da cirurgia em suas variáveis.

Objetivos: Avaliar os efeitos da correção cirúrgica da IM nas variáveis do TCPE realizados em bicicleta e em esteira rolante.

Métodos: Foram selecionados 72 pacientes com IM grave e submetidos à cirurgia corretiva da valvopatia, sendo nestes realizados ecocardiograma (ECO) e TCPE \pm 30 dias antes da cirurgia, e de seis a 12 meses após a cirurgia.

Resultados: A média das idades foi de 49,9 $\pm 16,9$ anos. Houve predominância do sexo masculino, da etiologia degenerativa e da classe funcional I ou II pela NYHA em 41 pacientes $(56,9 \%), 50$ pacientes $(69,4 \%)$ e 51 pacientes $(70,8 \%)$, respectivamente. $O$ reparo mitral foi realizado em 47 pacientes $(65,3 \%)$ e a troca valvar em 25 pacientes. Após a cirurgia houve redução do volume atrial esquerdo (VAE), de 124,4 $\pm 58,9 \mathrm{ml}$ para 76,4 \pm $34,0 \mathrm{ml}(\mathrm{p}<0,001)$, do volume diastólico final do VE (VDFVE), de 151,7 \pm $35,7 \mathrm{ml}$ para $109,6 \pm 42,8 \mathrm{ml}(p<0,001)$, do volume sistólico final do VE (VSFVE), de $47,6 \pm 18,0$ para $43,9 \pm 27,1 \mathrm{ml}(p<0,001)$, da fração de ejeção (FE), de $69,1 \pm 6,3$ para $61,30 \pm 8,2 \%(p<0,001)$ e da pressão sistólica em artéria pulmonar (PSAP), de 46,7 $\pm 15,9$ para $35,4 \pm 12,5 \mathrm{~mm} \mathrm{Hg}(\mathrm{p}<0,001)$. Também foi observado uma diminuição do consumo de oxigênio (VO2) pico em ambas as modalidades de exercício após a cirurgia (bicicleta: de $1356 \pm$ 506 para $1269 \pm 408 \mathrm{~mL} \cdot \mathrm{min}-1, \mathrm{p}=0,015$; esteira: de $1618 \pm 579$ para $1536 \pm$ $447 \mathrm{~mL}$.min-1, $\mathrm{p}=0,06$ ), assim como a variável OUES (bicicleta: de $1530 \pm$ 516 para $1414 \pm 415, p=0,001$; esteira: de $1761 \pm 585$ para $1691 \pm 508$, $\mathrm{p}=0,050)$. $\mathrm{O}$ pulso de oxigênio $(\mathrm{O} 2)$ aumentou após a cirurgia apenas no TCPE realizado em esteira, de 10,7 $\pm 4,0$ para 11,5 $\pm 3,2 \mathrm{~mL}$ bat $^{-1}(\mathrm{p}=0,023)$. No TCPE realizado em esteira, a diferença percentual do OUES apresentou significativa correlação negativa com a diferença percentual do VDFVE $(r=-$ $0,277 ; p=0,034)$, do $\operatorname{VAE}(r=-0,275 ; p=0,035)$ e da PSAP $(r=-0,282 ; p=0,041)$, respectivamente. No TCPE realizado na bicicleta, a diferença percentual do OUES revelou correlação negativa apenas com a diferença percentual da PSAP $(r=-0,359 ; p=0,010)$.

Conclusão: A cirurgia valvar mitral, não determinou aumento do VO2 pico e do OUES apesar da melhora sintomática avaliada pela classe funcional 
(NYHA). Entretanto, a variável OUES apresentou correlação negativa com parâmetros ecocardiográficos de remodelação cardíaca. O TCPE é uma ferramenta útil e promissora, podendo auxiliar na conduta médica em pacientes com IM.

Descritores: Insuficiência da Valva Mitral. Sintomas. Exercício. Cirurgia Cardíaca. 


\section{SUMMARY}

Della Togna, DJ. Effect of Surgical Correction on Cardiopulmonary Exercise Testing in Patients with Organic Mitral Regurgitation [Thesis]. São Paulo: Institute Dante Pazzanese of Cardiology, University of São Paulo; 2012. 130p.

Introduction: Chronic organic Mitral Regurgitation (MR) has a complex physiopathology. The symptoms of heart failure and functional capacity worsening may appear too late, when a left ventricle (LV) contractile dysfunction already exists. The cardiopulmonary exercise testing (CPET) may objectively assess the functional capacity and helps in the medical conduct concerning patients with MR, but little is known about the surgery effect on its variables.

Objectives: To evaluate the effects of the MR surgical correction on the variables related to the CPET performed on stationary bicycle and treadmill.

Methods: A total of 72 patients with severe MR were selected and underwent a surgery for valvulopathy correction and an echocardiogram (ECHO) and CPET were performed around 30 days before the surgery and then from six to 12 months after the surgery.

Results: The patients were aged an average $49.9 \pm 16.9$ years. There was a predominance of male patients [ 41 patients $(56.9 \%)$ ], degenerative etiology [50 patients $(69.4 \%)$ ] and NYHA I or II functional class [51 patients $(70.8 \%)$ ]. Forty-seven (47) patients (65.3\%) had their mitral valves repaired and 25 patients had them replaced. After the surgery, there was a reduction in the left atrial volume (LAV) from $124.4 \pm 58.9 \mathrm{ml}$ to $76.4 \pm 34.0 \mathrm{ml}(\mathrm{p}<0.001)$, in the LV end-diastolic volume (LVEDV) from $151.7 \pm 35.7 \mathrm{ml}$ to $109.6 \pm 42.8 \mathrm{ml}$ $(p<0.001)$, in the LV end-systolic volume (LVESV) from $47.6 \pm 18.0$ to $43.9 \pm$ $27.1 \mathrm{ml}(\mathrm{p}<0.001)$, in the ejection fraction $(\mathrm{EF})$ from $69.1 \pm 6.3$ to $61.30 \pm$ $8.2 \%(p<0.001)$ and in the pulmonary artery systolic pressure (PASP) from $46.7 \pm 15.9$ to $35.4 \pm 12.5 \mathrm{~mm} \mathrm{Hg}(p<0.001)$. A reduction in the peak consumption of oxygen (VO2) was also observed in both types of exercise testing after the surgery (stationary bicycle: from $1356 \pm 506$ to $1269 \pm 408$ $\mathrm{mL}$.min-1, $\mathrm{p}=0.015$; treadmill: from $1618 \pm 579$ to $1536 \pm 447 \mathrm{~mL} \cdot \mathrm{min}-1$, $\mathrm{p}=0.06$ ), as well as in the variable Oxygen Uptake Efficiency Slope (OUES) (stationary bicycle: from $1530 \pm 516$ to $1414 \pm 415, p=0.001$; treadmill: from $1761 \pm 585$ to $1691 \pm 508, p=0.050)$. Pulse oxymetry readings (O2) increased after the surgery only for the CPET performed on the treadmill from $10.7 \pm 4.0$ to $11.5 \pm 3.2 \mathrm{~mL}$. heartbeat $-1 \quad(p=0.023)$. For the CPET performed on the treadmill, the OUES percent difference showed significant negative correlation with the percent difference of LVEDV $(r=-0.277$; $p=0.034)$, LAV ( $r=-0.275 ; \quad p=0.035)$ and PASP $(r=-0.282 ; p=0.041)$, respectively. For the CPET performed on the stationary bicycle, the percent difference of the OUES showed a negative correlation only with the percent difference of PSAP $(r=-0.359 ; p=0.010)$. 
Conclusion: The mitral valve surgery caused no increase in the peak VO2 or OUES despite the symptomatic improvement as assessed through the functional class (NYHA). However, the variable OUES showed negative correlation with the echocardiographic parameters of cardiac remodeling. The CPET is a useful and promising tool, and may help in the medical conduct concerning patients with MR.

Descriptors: Chronic Mitral Regurgitation. Symptoms. Exercise. Surgical Cardiac 
Introdução 


\subsection{Insuficiência mitral}

A insuficiência mitral (IM) caracteriza-se por uma lesão valvar de grande interesse na prática clínica devido à sua alta prevalência ${ }^{1}$, sendo a lesão moderada ou grave, a doença valvar mais frequente nos Estados Unidos da América. Sua prevalência aumenta com a idade e estima-se em cinco milhões de indivíduos com IM em 2030². Na Europa ocupa o segundo lugar das valvopatias com indicação cirúrgica ${ }^{3}$. Apesar de o declínio da doença reumática ao redor do mundo, nos países, onde a doença reumática é endêmica, a IM, geralmente, acomete indivíduos adultos jovens e é um grave problema de Saúde Pública ${ }^{4}$. A distribuição da doença cardíaca reumática é universal e com marcadas diferenças entre os diversos países, sendo a principal causa de cardiopatia adquirida em crianças e adultos jovens nos países em desenvolvimento ${ }^{5,6}$. No Brasil, não há referências sobre a incidência de insuficiência mitral, mas sabe-se que a cardiopatia reumática crônica tem prevalência estimada em 1-7 casos por 1000 habitantes e é a causa mais frequente de cirurgia cardíaca na população adulta $^{7,8}$.

Nas últimas décadas, ocorreu notável progresso no entendimento da patofisiologia, manejo clínico e tratamento da IM. Os avanços nos métodos 
diagnósticos, especialmente a Doppler-ecocardiografia e o aperfeiçoamento das técnicas cirúrgicas, com possibilidade cada vez maior de preservação do aparato valvar e da função ventricular esquerda, vem despertando interesse crescente nessa doença. A possibilidade de reparo valvar cirúrgico com sucesso favorece a indicação cirúrgica mais precoce, inclusive em pacientes assintomáticos. Entretanto, ainda permanece controverso o momento ideal de indicação cirúrgica nos pacientes com IM assintomáticos e função sistólica ventricular esquerda normal, devido à falta de evidências definitivas que comprovem a superioridade da cirurgia em relação ao seguimento Clínico ${ }^{9-12}$

\subsection{Etiologia da insuficiência mitral}

O aparato valvar mitral é uma estrutura complexa que depende do funcionamento adequado e coordenado das cúspides, das estruturas subvalvares (cordas tendíneas e músculos papilares), do anel mitral e paredes do átrio esquerdo $(\mathrm{AE})$, ventrículo esquerdo e da aorta próxima à valva mitral (VM). Qualquer anormalidade em um ou mais destes componentes pode levar à IM, definida como a presença de refluxo do ventrículo esquerdo para o $\mathrm{AE}$. Baseado no seu mecanismo gerador, a IM pode ser classificada em primária ou orgânica e secundária ou funcional.

A IM primária é devida ao acometimento intrínseco das cúspides ou cordas tendíneas. Os principais exemplos incluem a degeneração mixomatosa resultando no prolapso da valva mitral (PVM) com ou sem 
ruptura de cordas tendíneas, doença reumática levando a espessamento e retração das cúspides, fusão e encurtamento das cordas tendíneas e a endocardite infecciosa com deformação e destruição do aparato mitral. Também podemos citar como outras causas de IM primária a calcificação progressiva do anel mitral, principalmente na população idosa, as doenças inflamatórias incluindo as colagenoses, a IM induzida por medicação ou radiação, as congênitas (fissura ou Cleft das cúspides) e as traumáticas com ruptura das cúspides ou cordas tendíneas. A IM degenerativa normalmente é relacionada ao PVM e raramente à calcificação isolada do anel mitral, comum na população idosa.

A IM secundária ou funcional ocorre devido ao remodelamento do ventrículo esquerdo, com ou sem dilatação do anel mitral e a integridade anatômica das cúspides é mantida. Ela ocorre em mais de $40 \%$ dos pacientes com insuficiência cardíaca (IC) devido à miocardiopatia dilatada ${ }^{13}$ e contribui para o círculo vicioso que integra a sobrecarga de volume levando à dilatação do ventrículo esquerdo, com consequente piora da IM e da insuficiência cardíaca. Vários fatores estão envolvidos como, por exemplo, mudanças na geometria e na função global e regional do ventrículo esquerdo, alterações na geometria do anel mitral, aumento da distância entre os músculos papilares, mobilidade anormal das cúspides e mau alinhamento dos músculos papilares, levando à tração das cordas tendíneas e coaptação inadequada das cúspides.

A IM isquêmica é considerada um subgrupo da IM funcional e sua gravidade está relacionada à extensão da área de acinesia ou discinesia do 
ventrículo esquerdo. Também pode ser de etiologia orgânica se houver ruptura de músculo papilar.

\subsection{Patofisiologia da insuficiência mitral}

O grau de regurgitação mitral depende da área do orifício de regurgitação $(A O R)$, do gradiente de pressão entre o ventrículo esquerdo e o AE e da complacência atrial esquerda ${ }^{14}$.

A IM primária crônica é caracterizada por sobrecarga pura de volume de câmaras esquerdas e aumento gradual e adaptativo do $A E$ e ventrículo esquerdo. Sua evolução é lenta e a hipertrofia excêntrica, com adição de sarcômeros em série e alongamento da fibra miocárdica é o mecanismo compensatório básico para manutenção do estresse sistólico da parede do ventrículo esquerdo. O débito cardíaco efetivo anterógrado também se mantém normal à custa de ajustes neuro-humorais e alterações da pré e da pós-carga. O acréscimo de sarcômeros, na fase compensada, aumenta a extensão e a velocidade de encurtamento da fibra miocárdica, aumentando a força de contração do ventrículo esquerdo, independente do mecanismo de Frank-Starling, mantendo o fluxo efetivo anterógrado e a pressão sistólica final do ventrículo esquerdo dentro da normalidade.

A piora da IM crônica e orgânica ocorre por acentuação da lesão valvar e pela dilatação do ventrículo esquerdo e anel mitral, resultando em

aumento do volume regurgitante de aproximadamente $5 \mathrm{a} 7 \mathrm{ml}$ por $\operatorname{ano}^{15} \mathrm{e}$ 
contribui para o mecanismo gerador e autossustentável da insuficiência mitral.

A dilatação progressiva do $\mathrm{AE}$, cavidade de maior complacência, proporciona atenuação do aumento de pressão no seu interior, minimizando o impacto na circulação pulmonar. Em pacientes com IM grave mais de 50\% do volume regurgitante reflui para o átrio esquerdo antes da abertura dos folhetos da valva aórtica e pode representar cerca de $20 \%$ do total do volume ventricular ${ }^{16}$. O aumento atrial esquerdo pode levar a arritmias atriais, principalmente à fibrilação atrial (FA).

A contratilidade miocárdica, definida como a velocidade ou capacidade inerente de encurtamento da fibra miocárdica, habitualmente, é preservada mesmo em fases avançadas da doença. A queda da contratilidade miocárdica é considerada um forte marcador de resultados adversos, tanto em pacientes submetidos à cirurgia para correção da valvopatia como naqueles em seguimento clínico.

A fase compensada da IM crônica grave é caracterizada por aumento da pré-carga, manutenção ou leve redução da pós-carga e contratilidade miocárdica do ventrículo esquerdo normal. Nesta fase, em geral, o paciente encontra-se assintomático ou com poucos sintomas.

A fase descompensada da doença é caracterizada por grande aumento e hipertrofia inadequada do ventrículo esquerdo, levando à disfunção contrátil e elevação das pressões de enchimento. Mesmo nessa fase adiantada, a presença de sintomas não é um forte marcador de redução 
da contratilidade miocárdica e os pacientes com IM podem permanecer assintomáticos por longos períodos ${ }^{17}$.

O intervalo médio do diagnóstico de IM até o início dos sintomas de IC pode chegar a 16 anos, com os sintomas aparecendo numa frequência de 2 a $4 \%$ por ano ${ }^{18}$, dependendo da gravidade e etiologia da IM.

A transição da fase compensada para a descompensada ocorre de forma insidiosa, sem nenhum marcador clínico ou laboratorial evidente. Entretanto, as condições de carga ainda favoráveis, frequentemente, mantêm a fração de ejeção ( $F E)$ em faixa considerada normal-baixa (50 a $60 \%$ ) apesar da presença de significativa disfunção contrátil ${ }^{19-21}$ Ainda, não se sabe com exatidão quais os mecanismos que levam ao declínio da função ventricular, mas supõe-se existirem fatores hemodinâmicos e celulares desconhecidos $^{22}$. A progressão da doença, se não modificada pela correção cirúrgica, pode levar à queda irreversível da contratilidade miocárdica.

Os índices de avaliação da função sistólica do ventrículo esquerdo, normalmente derivados do ecocardiograma, são dependentes das condições de pré e pós-carga, como por exemplo, a FE e a fração de encurtamento do ventrículo esquerdo. Na fase compensada da IM crônica grave, é comum a FE apresentar valores altos, devido a pré-carga elevada, mesmo em presença de redução da contratilidade do ventrículo esquerdo ${ }^{19}$. Após a correção cirúrgica da IM, a FE pode diminuir aproximadamente 10 unidades $^{23,24}$, permanecendo inalterados o volume e o stress sistólico final do ventrículo esquerdo ${ }^{24}$. 
A dificuldade de se obter índices de avaliação da função ventricular de fácil aplicabilidade clínica e independente das condições de carga, torna complicada a decisão do momento ideal de indicação cirúrgica, motivando a pesquisa de novos métodos ${ }^{25}$.

\subsection{Manejo clínico e indicação cirúrgica na insuficiência mitral}

O tratamento definitivo e considerado "padrão-ouro" da IM crônica e grave é a correção cirúrgica. De modo geral, a indicação de cirurgia tem por objetivo o alívio dos sintomas, o aumento da expectativa de vida com baixos índices de complicação pós-operatória e a preservação da função ventricular esquerda.

As modalidades de tratamento cirúrgico da IM são a troca valvar, com ou sem preservação do aparato subvalvar e o reparo. Apesar dos bons resultados em longo prazo da troca valvar, uma série de complicações está associada aos substitutos valvares como trombose de próteses, sobretudo nas mecânicas, hemorragia secundária ao uso de anticoagulante e degeneração das biopróteses. Por outro lado, o reparo mitral tem algumas vantagens em relação à troca valvar, incluindo a não obrigatoriedade de terapia antitrombótica em pacientes com ritmo sinusal, preservação da integridade ventrículo-valvar, manutenção da função ventricular esquerda e menor mortalidade no pós-operatório imediato e tardio. Apresenta ainda excelentes resultados em longo prazo e uma expectativa de vida muito próxima à da população geral ${ }^{26-37}$. Esses bons resultados do reparo, aliado 
às dificuldades existentes em se avaliar a contratilidade miocárdica de forma confiável, são argumentos convincentes para a indicação cirúrgica precoce realizada em pacientes assintomáticos ${ }^{38}$. Devido a isso, o reparo mitral tem sido a modalidade cirúrgica de escolha em alguns centros, onde se observa que a sua frequência aumentou de 18,9\% em 1998 para 45,8\% dos procedimentos mitrais em $2005^{39}$, sendo atualmente o procedimento cirúrgico mais, frequentemente, realizado ${ }^{40}$.

Atualmente, os pilares básicos de indicação cirúrgica na IM são a presença de sintomas e disfunção ventricular esquerda, ambos reconhecidos marcadores de resultados pós-operatórios adversos. Apesar dos avanços no manejo clínico e nos métodos diagnósticos, muitos pacientes são encaminhados tardiamente para a cirurgia e evoluem com disfunção irreversível do VE no período pós-operatório. Em paciente sintomático, a indicação cirúrgica é evidente, entretanto, no assintomático o momento ideal de cirurgia permanece controverso, por não se conhecer, exatamente, a partir de que ponto os benefícios da cirurgia superariam os seus riscos 9 .

A presença de disfunção ventricular esquerda em pacientes com IM também é um importante marcador prognóstico pós-operatório, com aumento da mortalidade tardia de até $180 \%$ para aqueles com FE préoperatória menor que $50 \%{ }^{41}$.

Os pacientes sintomáticos com IM apresentam uma maior mortalidade após a cirurgia, quando comparados com os assintomáticos, apesar de apresentarem melhora dos sintomas, segundo observação de Tribouilloy et 
$a^{42}$. Nesse estudo, com resultados em longo prazo, a sobrevida pósoperatória para os pacientes em CF NYHA I/II foi significativamente maior que a observada naqueles em CF III/IV (76 $\pm 5 \%$ "versus" $48 \pm 4 \%$, $p<0,0001)$ e a mortalidade operatória significativamente menor $(0,5 \%$ “versus" $5,4 \%, p=0,003)$.

Outros marcadores de pior prognóstico clínico têm sido avaliados em pacientes assintomáticos. Em estudo de Enriquez-Sarano et a $\mathrm{f}^{43}$, a presença de uma área do orifício de regurgitação $(A O R) \geq 4,0 \mathrm{~mm}^{2}$ foi um forte marcador de resultados clínicos adversos com uma sobrevida em cinco anos inferior a esperada para a população geral de acordo com o banco de dados dos Estados Unidos - "U.S.Census data" (58 $\pm 9 \%$ vs 78\%, $p=0,03$ ). Quando comparados aos pacientes com AOR mitral $<2,0 \mathrm{~mm}^{2}$, apresentaram um maior risco de morte cardíaca (razão de risco de 5,21; Cl 95\%, 1,98 a 14,40, p<0,01) e eventos cardíacos (razão de risco de 5,66; CI 95\%, 3,07 a 10,56, $\mathrm{p}<0,01)$. Em estudo de Yamano et $a f^{44}, 174$ pacientes com IM isolada e submetidos ao reparo cirúrgico, o volume regurgitante mitral $\geq 80 \mathrm{ml}$ foi 0 único marcador independente e significativo $(p<0,001)$ de disfunção ventricular esquerda ( $\mathrm{FE}<50 \%$ ) no pós-operatório, presente em 14 pacientes $(20 \%)$.

Atualmente, a recomendação classe I de indicação cirúrgica pelas diretrizes da American College of Cardiology (ACC) e American Heart Association (AHA $)^{45}$ é considerada para pacientes sintomáticos em CF II, III ou IV da New York Heart Association (NYHA), sem disfunção ventricular esquerda grave ou pacientes assintomáticos com disfunção contrátil leve a 
moderada do ventrículo esquerdo (FE de $30 \%$ a $60 \%$ ou dimensão sistólica final do ventrículo esquerdo $\geq 40 \mathrm{~mm}$ ). Essa recomendação é mantida mesmo naqueles pacientes, nos quais os sintomas melhoraram com o tratamento clínico, especialmente se houver ruptura das cordas tendíneas ${ }^{46}$. Segundo as mesmas diretrizes, os pacientes assintomáticos com função ventricular esquerda normal podem apresentar recomendação classe Ila, se houver alta possibilidade de reparo (acima de 90\%) sem IM residual e realizados em centros de cardiologia com experiência cirúrgica comprovada, FA ou hipertensão arterial pulmonar (HAP, pressão sistólica em artéria pulmonar > $50 \mathrm{~mm} \mathrm{Hg} \mathrm{em} \mathrm{repouso} \mathrm{ou} \mathrm{>} 60$ mm Hg após o exercício).

Já as diretrizes da Sociedade Europeia de Cardiologia recomendam a cirurgia em pacientes com IM assintomática apenas na presença de marcadores de mau prognóstico como disfunção ventricular esquerda, FA e $\mathrm{HAP}^{47}$.

Portanto, como já descrito anteriormente, o momento ideal de indicação cirúrgica nos pacientes com IM grave, assintomático e com boa função ventricular esquerda, permanece controverso e de difícil decisão ${ }^{48}$. Somente ensaios clínicos prospectivos e randomizados, ainda não realizados, poderiam ajudar a solucionar esta questão.

\subsection{Avaliação da Classe Funcional na insuficiência mitral}

A dispneia é um dos principais sintomas de doenças cardíacas e pulmonares e é definida como uma sensação de desconforto da 
respiração ${ }^{49}$. Ela também pode ser observada em indivíduos saudáveis durante e após atividade física, especialmente naqueles que não a praticam, sedentários ou com excesso de peso. Em 1928, a NYHA publicou uma classificação dos pacientes com doenças cardíacas baseada no prognóstico e na gravidade da doença e, posteriormente, foi revisada em sete edições, sendo a última liberada em março de $1994^{50}$. Esta classificação leva em consideração a avaliação subjetiva dos sintomas e a quantificação objetiva da gravidade da doença e, é um forte marcador independente de sobrevida em pacientes com insuficiência cardíaca crônica ${ }^{51}$.

A avaliação dos sintomas em pacientes com IM crônica também apresenta consideração especial, pois pode aparecer somente nas fases tardias da doença e com comprometimento miocárdico irreversível. Além disso, estudos prévios não têm demonstrado forte correlação entre os sintomas e a função ventricular esquerda, mesmo nas patologias cardíacas em que as alterações das condições de carga impostas ao ventrículo esquerdo não são tão evidentes quanto na IM crônica. A avaliação adequada da CF também depende de diversos fatores, como a capacidade de expressão e percepção na relação médico-paciente, o nível de sedentarismo, comum no idoso, a autolimitação ao exercício imposta pela doença, por vezes imperceptível e condições de comorbidades como, por exemplo, a obesidade ou problemas ortopédicos limitantes da prática de atividade física.

Como foi visto, em pacientes com IM é importante uma avaliação adequada da CF para decisão terapêutica. $O$ teste ergométrico (TE) e o 
teste cardiopulmonar de exercício (TCPE) avaliam a capacidade de exercício de uma forma objetiva, minimizando os aspectos subjetivos da anamnese.

Em paciente com doença cardíaca valvar, O TE pode fornecer informações valiosas sobre a capacidade funcional e resposta hemodinâmica ao exercício, sobretudo no paciente com lesão grave assintomático ou com sintomas atípicos e naqueles que limitam sua atividade física. Nos pacientes com IM grave assintomática, a demonstração pelo TE de capacidade funcional reduzida ou a queda da pressão arterial sistólica durante o exercício podem auxiliar o cardiologista na decisão terapêutica. A avaliação da capacidade funcional através do TE no paciente com valvopatia é considerado recomendação classe lla, com nível de evidência B, pela Sociedade Brasileira de Cardiologia ${ }^{52}$.

Gohlke-Barwolf et $a f^{53}$, realizaram o teste ergométrico em 1270 pacientes submetidos à troca valvar aórtica ou mitral isolada e que participaram de um programa de reabilitação pós-operatória. Os pacientes submetidos à troca valvar mitral apresentaram uma significativa menor capacidade de exercício pós-operatório comparado com os pacientes submetidos à troca valvar aórtica.

Entretanto, ainda permanece incerta a real indicação do TE em pacientes com IM, mesmo em assintomáticos e sendo raramente realizado.

O teste cardiopulmonar de exercício (TCPE) é um exame complementar que traz informações diagnósticas e prognósticas de diversas cardiopatias, possibilitando um melhor manejo clínico ou cirúrgico dentro do continuum cardiovascular. 
Inicialmente utilizado como instrumento de pesquisa, é um método não invasivo que combina as informações obtidas da ergometria convencional com a análise direta dos gases expirados. Aplicado na prática clínica desde a década de 1980, o TCPE permite uma avaliação quantitativa e objetiva da capacidade funcional, estratifica o risco em pacientes com insuficiência cardíaca e avalia a resposta terapêutica ${ }^{54}$. Trata-se de metodologia que tem alterado profundamente a avaliação funcional dos pacientes e fornecendo descritores altamente reproduzíveis da capacidade de exercício, como por exemplo, o consumo pico de oxigênio $\left(\mathrm{VO}_{2} \text { pico }\right)^{55,56}$. A avaliação combinada do teste ergométrico convencional e das medidas diretas a cada ciclo respiratório (respiração a respiração) do consumo de oxigênio $\left(\mathrm{VO}_{2}\right)$, produção de gás carbônico $\left(\mathrm{VCO}_{2}\right)$ e da ventilação $(\mathrm{VE})$ fornecem uma análise detalhada e integrada das respostas ao exercício envolvendo os sistemas cardiovascular, pulmonar, hematopoiético, muscular e neuropsicológico. As medidas diretas do $\mathrm{VO}_{2}, \mathrm{VCO}_{2}$ e da $\mathrm{VE}$, analisadas individualmente ou associadas em diversas combinações, definem as chamadas variáveis do TCPE, permitindo, durante o exercício progressivo máximo, identificar suas diferentes fases metabólicas e alterações hemodinâmicas.

A finalidade principal do TCPE em paciente portador de valvopatia é avaliar objetivamente a presença de sintomas atípicos, sua capacidade funcional e a repercussão hemodinâmica.

Em estudo de Bissessor et $a^{57}$, realizado em 45 pacientes com doença valvar complexa e em CF I ou II da NYHA, os valores do $\mathrm{VO}_{2}$ pico 
foram significativamente menores que os do grupo controle composto de 15 voluntários saudáveis e não treinados $\left(16 \pm 5,9 \mathrm{~mL} \cdot \mathrm{kg}^{-1} \cdot \mathrm{min}^{-1}\right.$ e $31,4 \pm 5,9$ $\left.\mathrm{mL} \cdot \mathrm{kg}^{-1} \cdot \mathrm{min}^{-1}, \mathrm{p}=0,0001\right)$. Neste estudo, ao utilizar análise de regressão multivariada, o $\mathrm{VO}_{2}$ pico e a inclinação da reta de regressão linear entre a ventilação e a produção de gás carbônico $\left(\mathrm{NE} / \mathrm{VCO}_{2}\right.$ slope), foram fortes marcadores de resultados adversos como reparo ou troca valvar, morte e insuficiência cardíaca. Os autores concluem que o declínio do $\mathrm{VO}_{2}$ pico poderia identificar o momento mais adequado de intervenção cirúrgica e evitar resultados adversos.

O papel do TCPE na avaliação objetiva do grau funcional e como indicador prognóstico na IM foi avaliado por Kim HJ et $a^{58}$ em 31 pacientes submetidos a reparo valvar. O TCPE realizado antes e um ano após a cirurgia não mostrou melhora significativa nos valores do $\mathrm{VO}_{2}$ pico $\left(\mathrm{VO}_{2}\right.$ pico pré-operatório de $23,1 \pm 6,2 \mathrm{~mL} \cdot \mathrm{kg}^{-1} \cdot \mathrm{min}^{-1}$ e pós-operatório de $22,9 \pm 6,4$ $\left.\mathrm{mL} \cdot \mathrm{kg}^{-1} \cdot \mathrm{min}^{-1}, \mathrm{p}=0,82\right)$, entretanto, houve redução significativa naqueles que evoluíram com IM residual pós-operatória de grau discreto ou moderado (14 pacientes, $\mathrm{VO}_{2}$ pico pré-operatório de $22,7 \pm 6,4 \mathrm{~mL} \cdot \mathrm{kg}^{-1} \cdot \mathrm{min}^{-1}$ e pósoperatório de 21,0 $\left.\pm 6,3 \mathrm{~mL} \cdot \mathrm{kg}^{-1} \cdot \mathrm{min}^{-1}, \mathrm{p}=0,04\right)$. Os pacientes com maior $\mathrm{VO}_{2}$ pico pré-operatório $\left(\geq 18,5 \mathrm{~mL} \cdot \mathrm{kg}^{-1} \cdot \mathrm{min}^{-1}\right)$ tiveram uma significativa menor CF da NYHA no pós-operatório quando comparados aos pacientes com menores valores do $\mathrm{VO}_{2}$ pico $\left(<18,5 \mathrm{~mL} \cdot \mathrm{kg}^{-1} \cdot \mathrm{min}^{-1}, \mathrm{p}=0,03\right)$. Os autores deste estudo sugerem que o TCPE pode ser útil em determinar o momento da cirurgia e o valor pré-operatório do $\mathrm{VO}_{2}$ pico de $18,5 \mathrm{~mL} \cdot \mathrm{kg}^{-1} \cdot \mathrm{min}^{-1}$ poderia ser usado como marcador de melhora do grau funcional, porém ainda 
permanece incerto os motivos pelos quais o valor do $\mathrm{VO}_{2}$ pico diminui após a cirurgia em pacientes que evoluem com IM residual.

Em outro estudo realizado por Le Tourneau et $a \beta^{37}$, o TCPE, a angiografia por radionuclídeo e amostras de sangue para avaliação do estado neuro-hormonal foram obtidos antes e um ano (216 \pm 80 dias) após a cirurgia em 40 pacientes com IM não isquêmica (24 pacientes submetidos ao reparo com sucesso e 16 pacientes a troca valvar). Apesar da melhora da classe funcional avaliada pela NYHA, a performance de exercício não se modificou após a correção cirúrgica da IM $\left(\mathrm{VO}_{2}\right.$ pico de 19,3 $\pm 6,1 \mathrm{~mL} . \mathrm{kg}^{-}$ ${ }^{1} \cdot \mathrm{min}^{-1}$ para 18,5 $\pm 5,6 \mathrm{~mL} \cdot \mathrm{kg}^{-1} \cdot \mathrm{min}^{-1}$ e porcentagem alcançada do $\mathrm{VO}_{2}$ máx. predito de $79,5 \pm 18,2 \%$ para $76,8 \pm 16,9 \%$ ) em todos os pacientes, independente do tipo de cirurgia realizada. Também não houve diferenças entre os grupos, antes e após a cirurgia, na tolerância máxima ao exercício avaliado pelo pulso de $\mathrm{O}_{2}$ pico ou pela porcentagem do $\mathrm{VO}_{2}$ predito, assim como nos pacientes classificados de acordo com a FE pré-operatória $(<60 \%$ em 14 pacientes e $\geq 60 \%$ em 26 pacientes) com respeito à porcentagem do $\mathrm{VO}_{2}$ máx. predito pós-operatório $(74,2 \pm 16,5 \%$ versus $77,1 \pm 17,7 \%)$ ou $\mathrm{VO}_{2}$ máx. $\left(18,1 \pm 6,9 \mathrm{~mL} \cdot \mathrm{kg}^{-1} \cdot \min .1\right.$ versus $\left.18,1 \pm 4,7 \mathrm{~mL} \cdot \mathrm{kg}^{-1} \cdot \mathrm{min}^{-1}\right)$. Apesar de o tempo de exercício ser um parâmetro de menor acurácia que o $\mathrm{VO}_{2}$ para avaliar a capacidade objetiva de exercício, neste estudo houve uma leve, porém significativa redução no tempo médio de exercício após a cirurgia (600 \pm 247 seg. para $544 \pm 204$ seg., $p=0,02)$. Portanto, neste estudo prospectivo, a performance de exercício não melhorou na maioria dos 
pacientes dentro do primeiro ano de cirurgia valvar mitral para IM crônica não-isquêmica.

Vários fatores influenciam a capacidade de exercício no pósoperatório de cirurgia valvar e podemos citar o grau de restabelecimento da função ventricular esquerda e da redução da pressão em artéria pulmonar ${ }^{59}$. Também devemos salientar que as próteses valvares são estenóticas e em alguns casos, como, por exemplo, quando implantadas em anel aórtico pequeno, poderia produzir gradientes elevados e piorar a hemodinâmica e a capacidade de exercício.

A reversão pós-operatória para ritmo sinusal em pacientes com FA também pode influenciar a capacidade de exercício, como demonstrado por Tamai et $a^{60}$, que avaliaram 25 pacientes, sendo 18 com doença valvar e sete com defeito em septo atrial, submetidos à cirurgia valvar ou fechamento do defeito septal, e também ao procedimento de Maze. A reversão da arritmia para ritmo sinusal ocorreu em 23 pacientes e o teste cardiopulmonar de exercício (TCPE) realizado antes, e um, seis e 12 meses após a cirurgia, demonstrou que houve uma melhora da capacidade de exercício avaliada pelo consumo pico de oxigênio em fase tardia após a cirurgia e relacionada à melhora da resposta cronotrópica do nó sinusal ao esforço.

Como vimos, poucos estudos têm avaliado os efeitos da correção valvar mitral na capacidade de exercício, particularmente em pacientes com $\mathrm{IM}^{37,58}$. Além disso, permanece desconhecido se a preservação da função ventricular após a cirurgia de correção da valvopatia mitral melhora a capacidade funcional ${ }^{61,62}$ 
Apesar de 0 incremento de informações importantes, o TCPE permanece pouco entendido e subutilizado na prática clínica atual. Isto se deve, em grande parte, aos custos relacionados à captação e análise dos gases expirados e à falta de proficiência de pessoal habilitado na aplicação do teste e interpretação dos resultados.

Na IM orgânica e crônica, é provável que algumas variáveis do TCPE possam trazer informação diagnóstica e prognóstica, tornando-se ferramenta útil no entendimento de sua patofisiologia e auxiliando no momento mais adequado de indicação cirúrgica em pacientes assintomáticos, mas são necessários estudos adicionais para definir as indicações e os critérios de seleção de pacientes para o seu uso nesta população. 
Objetivos 
- Avaliar a influência da correção cirúrgica da IM crônica, orgânica grave e não isquêmica nas variáveis do teste cardiopulmonar de exercício.

- Correlacionar as medidas de remodelação cardíaca pós-operatória com variáveis do teste cardiopulmonar de exercício em pacientes com IM crônica, orgânica e não isquêmica submetidos à correção cirúrgica da valvopatia.

- Comparar as variáveis do teste cardiopulmonar de exercício após a cirurgia de troca valvar mitral e reparo mitral em pacientes com IM crônica, orgânica e não isquêmica. 
Métodos 
A presente investigação foi aprovada pelo Comitê de Ética do Instituto Dante Pazzanese de Cardiologia, em reunião de 04.01.2008, conforme o parecer $\mathrm{n}$ - 3596 . Todos os participantes assinaram o Termo de Consentimento Livre e Esclarecido (anexo A) após conhecimento do estudo e métodos a serem realizados.

\subsection{Seleção de Pacientes}

Foram selecionados pacientes consecutivos, com diagnóstico de IM crônica grave não isquêmica e com indicação de correção cirúrgica baseada nas diretrizes da $\mathrm{AHA} / \mathrm{ACC}^{45}$, acompanhados no ambulatório de préoperatório da Seção Médica de Valvopatias do Instituto Dante Pazzanese de Cardiologia, no período de agosto de 2008 a janeiro de 2011.

\subsubsection{Critérios de Inclusão}

Foram incluídos pacientes com diagnóstico de IM orgânica, crônica, grave, isolada, não isquêmica e com indicação cirúrgica, idade acima de 14 anos, capacidade de realizar o teste cardiopulmonar de exercício e que aceitaram o protocolo de estudo e o termo de consentimento informado. 


\subsubsection{Critérios de Exclusão}

a) Doença coronária concomitante;

b) Defeitos cardíacos congênitos associados;

c) Insuficiência mitral aguda;

d) Quadro clínico-laboratorial compatível com endocardite infecciosa ativa;

e) Doença reumática ativa;

f) Cirurgia cardíaca prévia;

g) Estenose mitral associada com área valvar $\leq 2,0 \mathrm{~cm}^{2}$;

h) Valvopatia aórtica de gravidade moderada ou grave;

i) Doença pulmonar moderada ou grave;

j) Doença da tireoide descompensada;

k) Insuficiência renal crônica estágio $\geq 3$ (clearance de creatinina < $60 \mathrm{ml} / \mathrm{min}$.)

I) Miocardiopatia dilatada de qualquer etiologia não relacionada à insuficiência mitral;

m) Incapacidade de realizar o teste ergométrico por qualquer motivo;

n) Anormalidades neurológicas ou musculares significativas;

o) Cirurgia recente ( $<8$ semanas) que impossibilite a realização do teste ergométrico;

p) Hipertensão arterial sistêmica não controlada;

q) Arritmias graves com risco de vida; 
r) Incapacidade de entendimento ou de realizar o protocolo;

s) Portador de marca-passo definitivo;

t) Janela ecocardiográfica inadequada.

\subsection{Desenho do Estudo}

Estudo prospectivo e observacional em que todos os pacientes foram acompanhados e avaliados consecutivamente antes e de seis a 12 meses após a cirurgia cardíaca, selecionados para realizar estudo ecocardiográfico, hemodinâmico e o TCPE, conforme o protocolo descrito a seguir.

\subsection{Tamanho amostral}

Por serem poucos os trabalhos na literatura que avaliam o TCPE na IM, o cálculo do tamanho da amostra deste estudo foi baseado no trabalho pioneiro de Kim HJ et al. $2004^{58}$, em que foi avaliado o papel do TCPE em 31 indivíduos com IM submetidos ao reparo mitral. O TCPE realizado antes e um ano após a cirurgia revelou queda significativa do $\mathrm{VO}_{2}$ nos pacientes que evoluíram com IM residual pós-operatória.

No presente estudo, procurou-se alcançar pelo menos 31 pacientes submetidos ao reparo mitral, além de serem incluídos também os pacientes submetidos à troca valvar, totalizando tamanho da amostra de no mínimo 62 pacientes. 


\subsection{Protocolo de avaliação dos pacientes}

Os pacientes selecionados foram submetidos a:
a) Anamnese e exame físico pré e pós-operatório;
b) Eletrocardiograma (ECG) de repouso convencional com 12 derivações pré e pós-operatório;
c) Exames sanguíneos pré e pós-operatório: glicemia de jejum, sódio, potássio, ureia, creatinina e hemograma;
d) Ecocardiografia transtorácica (ECO) pré e pós-operatório;
e) Cateterismo cardíaco: pré-operatório;
f) Teste cardiopulmonar de exercício na bicicleta e esteira ergométrica: pré e pós-operatório.

\subsubsection{Anamnese, exame físico, indicação cirúrgica e etiologia da insuficiência mitral}

Todos os pacientes incluídos neste protocolo de estudo foram avaliados clinicamente pelo investigador principal antes e de seis a 12 meses após a cirurgia. Foi verificada a história clínica, classe funcional obtida pela classificação da NYHA e o exame físico geral.

A etiologia da IM foi baseada na avaliação ecocardiográfica préoperatória, descrição da cirurgia e no anatomopatológico da valva mitral quando disponível. 


\subsubsection{Eletrocardiograma}

O eletrocardiograma (ECG) de repouso convencional de 12 derivações foi realizado em todos os pacientes no período pré-operatório e de seis a 12 meses após a cirurgia de correção da valvopatia e avaliados o ritmo cardíaco, sobrecarga de câmaras cardíacas, distúrbios de condução atrioventricular e arritmias cardíacas. Os laudos do ECG foram elaborados de acordo com a diretriz da Sociedade Brasileira de Cardiologia para interpretação do eletrocardiograma de repouso ${ }^{63}$.

\subsubsection{Exames laboratoriais sanguíneos}

Foram realizados os seguintes exames laboratoriais em todos os pacientes no período pré-operatório e de seis a 12 meses após a cirurgia: glicemia de jejum, sódio, potássio, ureia, creatinina e hemograma.

\subsubsection{Ecocardiografia trans-torácica}

Os pacientes foram submetidos ao estudo ecocardiográfico na Seção Médica de Ecocardiografia do Instituto Dante Pazzanese de Cardiologia, no período pré-operatório até 30 dias que antecederam a cirurgia cardíaca e no pós-operatório de seis meses a um ano após a cirurgia, pelo mesmo observador. Foi utilizado um aparelho Vivid 7 Dimension Cardiovascular Ultrasound (GE Healthcare ${ }^{\circledR}$, Milwaukee, WI, EUA), com capacidade para 
armazenamento digital de imagens, equipado com um transdutor setorial M4S.

Foi realizado estudo ecocardiográfico completo, incluindo modo unidimensional (modo-M), bidimensional, Doppler convencional (nas modalidades pulsátil, contínuo e colorido) e o Doppler tecidual, de acordo com as recomendações da American Society of Echocardiography ${ }^{14,64,65}$. Todos os videoclipes adquiridos incluíam pelo menos quatro batimentos cardíacos, com registro de uma derivação eletrocardiográfica contínua. Os exames foram gravados em CD ou DVD, no formato Raw Data para posterior leitura e realização das medidas em um sistema operacional compatível com o aparelho utilizado (Echo-PAC GE Ultrasound).

Os parâmetros ecocardiográficos medidos pelo modo $\mathrm{M}$ e bidimensional foram: diâmetros diastólico e sistólico final do ventrículo esquerdo, volumes diastólico e sistólico final do ventrículo esquerdo (Figura 1), espessura diastólica do septo e da parede ínferolateral do ventrículo esquerdo, índice de massa ventricular esquerda, diâmetro anteroposterior e índice volumétrico do átrio esquerdo (Figura 2). A fração de ejeção do ventrículo esquerdo foi estimada pelo método biplanar de Simpson, com aquisição das imagens na janela ecocardiográfica apical de quatro e duas câmaras.

Utilizou-se o Doppler contínuo para medir as velocidades de fluxo diastólico através da valva mitral. O gradiente diastólico transvalvar mitral máximo correspondeu a maior velocidade diastólica do fluxo mitral e o gradiente médio correspondeu a média de todas as velocidades e foram 
obtidos, automaticamente, a partir do tracejado do espectro de velocidades do Doppler contínuo. A área valvar mitral foi estimada pelo método do Pressure Half Time (PHT), no momento pré-operatório. No período pósoperatório, a área valvar foi obtida pelo método da equação de continuidade, exceto nos casos em que esta não poderia ser utilizada por questões técnicas, em que o PHT foi o método de escolha.

Para estimativa da pressão sistólica em artéria pulmonar (PSAP), foi medida a diferença de pressão entre o átrio direito $(A D)$ e o ventrículo direito (VD), nos casos em que havia regurgitação tricúspide.

O mapeamento do fluxo em cores foi realizado em todas as valvas cardíacas. Na valva mitral, em particular, ele tem grande importância para determinação da gravidade da insuficiência, permitindo a medida da área do jato regurgitante (JR), da largura da vena contracta (VC) e das medidas derivadas do método do fluxo de convergência proximal (PISA - proximal isovelocity surface area), ou seja: AOR e volume regurgitante (Figuras 3 e 4).

A determinação da gravidade da insuficiência mitral foi realizada por meio dos seguintes métodos:

- Relação da área do JR e área do AE.

- Medida de largura da VC obtida no corte para-esternal longitudinal e apical quatro câmaras, utilizando posicionamento adequado do foco e zoom.

- Medida da AOR, do volume e fração regurgitante através do método de PISA. 
- Presença de fluxo reverso em veia pulmonar superior direita.

Foram considerados com IM grave aqueles pacientes que apresentaram pelo menos duas das seguintes características: relação área do jato regurgitante e área do átrio esquerdo maior do que 40\%, uma das medidas da vena contracta maior do que $0,7 \mathrm{~cm}$, AOR maior que $0,4 \mathrm{~cm}^{2}$, volume regurgitante maior que $60 \mathrm{ml} / \mathrm{batimento} \mathrm{cardíaco} \mathrm{e} \mathrm{presença} \mathrm{de} \mathrm{fluxo}$ sistólico reverso no interior da veia pulmonar superior direita ${ }^{45}$.
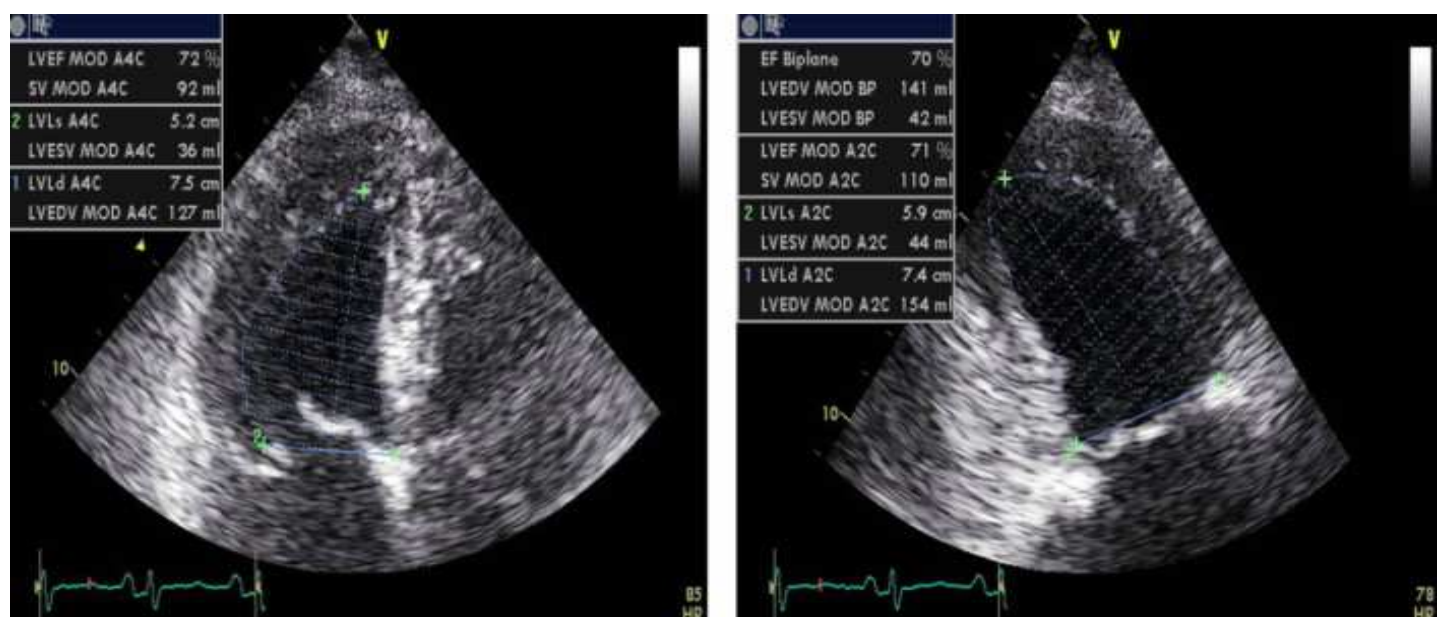

Figura 1 - As imagens exemplificam como foram feitas as medidas dos volumes do ventrículo esquerdo por meio da técnica ecocardiográfica de Simpson, em dois planos ortogonais. 

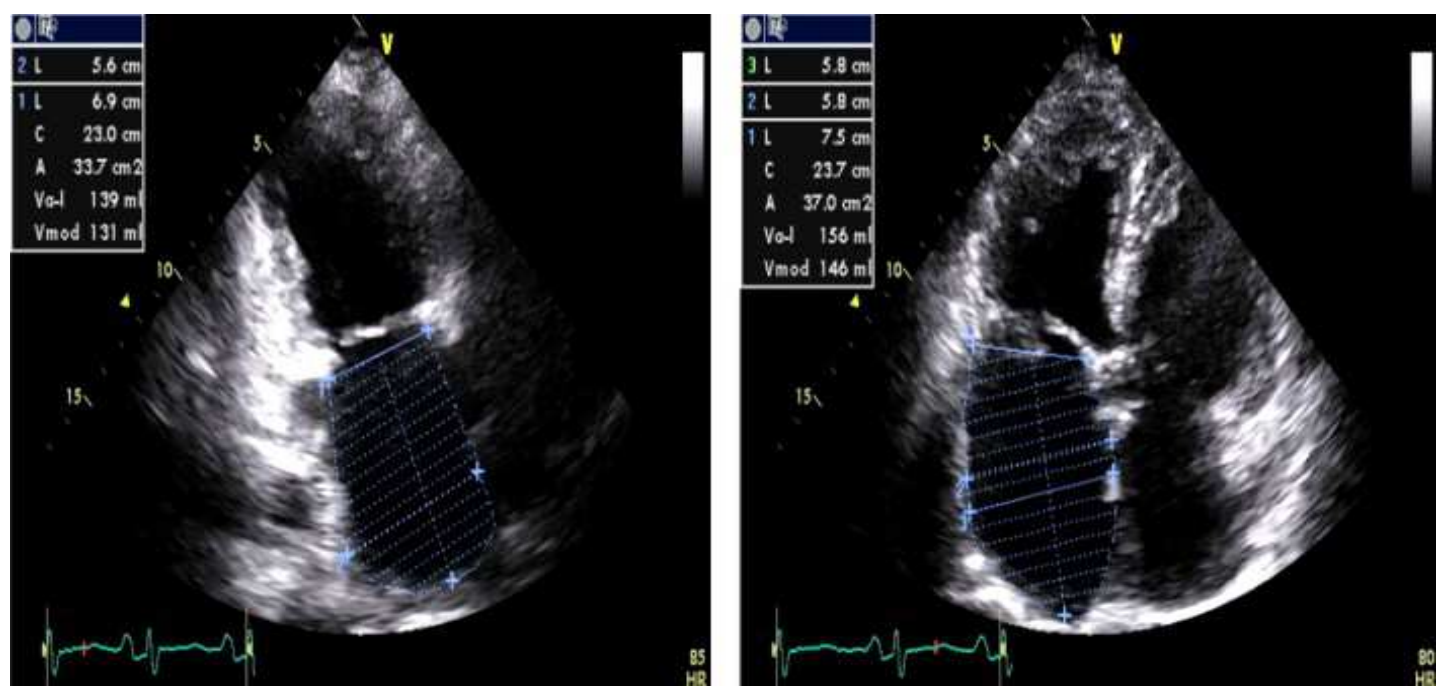

Figura 2 - Demonstração de como foram obtidas as medidas do volume sistólico do átrio esquerdo em dois planos ortogonais, por meio da técnica ecocardiográfica de Simpson.
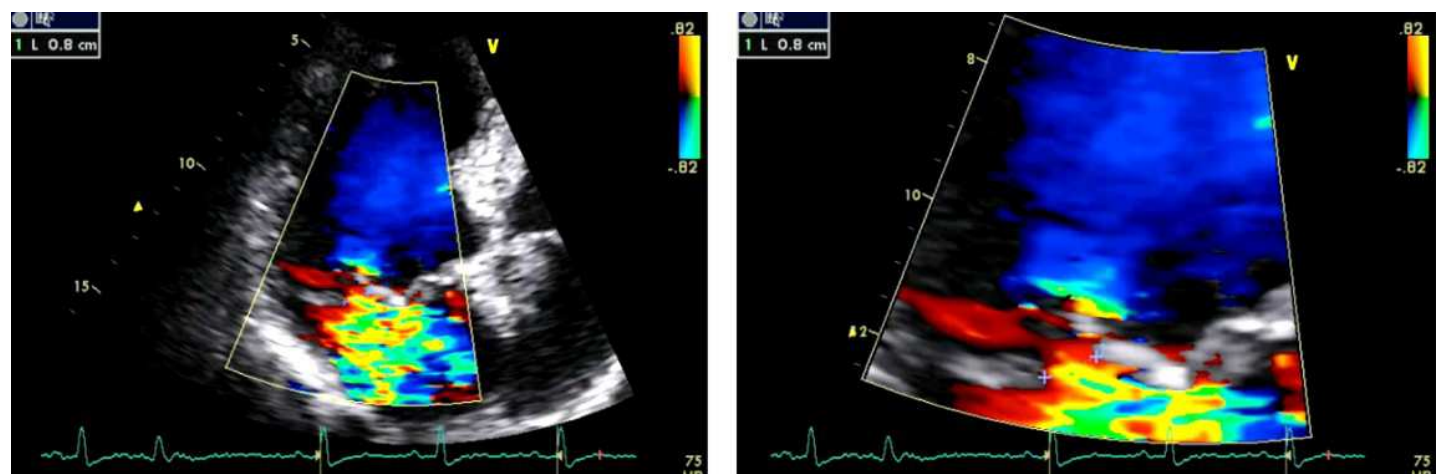

Figura 3 - As imagens demonstram a medida da vena contracta (região mais estreita do jato de insuficiência, logo abaixo da coaptação dos folhetos); este método ecocardiográfico foi utilizado para a quantificação da insuficiência valvar mitral. A imagem à direita é uma seção central ampliada da imagem à esquerda, com utilização de zoom. 

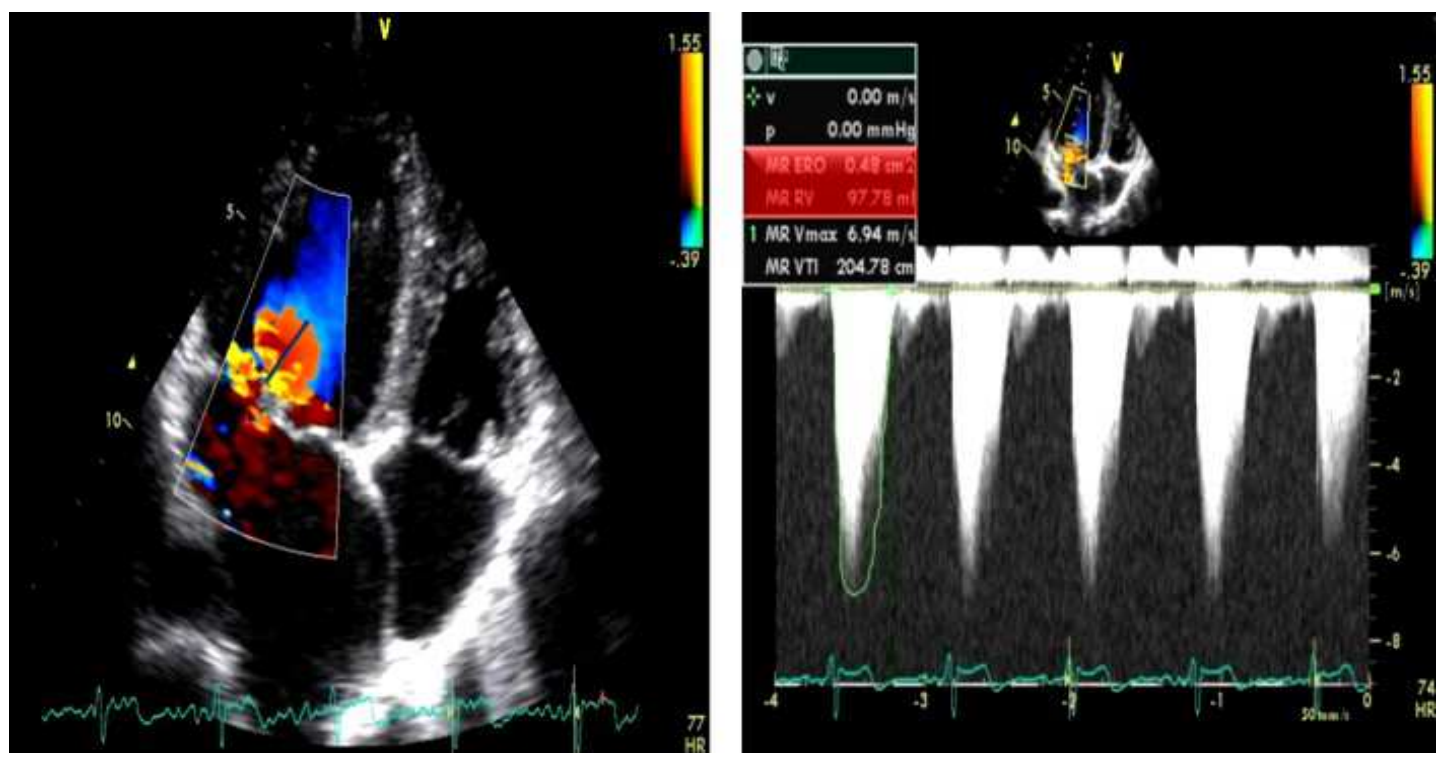

Figura 4 - As figuras representam a técnica ecocardiográfica de PISA (proximal isovelocity surface area), utilizada para a medida da área do orifício de regurgitação (AOR) e volume de regurgitação (VR), dados fundamentais para classificar a gravidade da insuficiência valvar mitral. À esquerda, observa-se a medida do raio da zona de convergência e à direita a medida da integral da velocidade do jato de refluxo.

\subsubsection{Teste cardiopulmonar de exercício}

Os TCPE foram realizados no Laboratório da Seção Médica de

Provas Funcionais do Serviço de Reabilitação Cardiovascular do Instituto

Dante Pazzanese de Cardiologia, previamente agendados para o período matutino após leve desjejum. Os pacientes foram orientados a não realizar atividade física cansativa na véspera e no dia do exame, dormir no mínimo oito horas na noite de véspera, não ingerir bebidas cafeinadas ou alcoólicas nas 24 horas que antecediam o exame, não fumar no dia do exame e manter toda a medicação regular. 
Os TCPE pré-operatórios foram agendados em até dois meses antes da cirurgia e os pós-operatórios entre seis e 12 meses após a cirurgia. Nas duas ocasiões, foram realizados testes em bicicleta ergométrica eletromagnética, de marca Corival e em esteira rolante da marca Inbramed, com intervalos entre sete e 30 dias.

Inicialmente, em repouso, foi realizada a espirometria no equipamento da marca Medical Graphics Corporation $\AA^{\circledR}$ (Minnesota, EUA) modelo Cardio $\mathrm{O}_{2}$. As manobras foram realizadas de acordo com os critérios da Sociedade Brasileira de Pneumologia e Tisiologia ${ }^{66}$ e determinados o volume expirado no primeiro segundo (VEF1), a capacidade vital forçada (CVF) e a ventilação voluntária máxima (VVM) necessária para o cálculo da reserva respiratória.

$\mathrm{Na}$ sequência, no mesmo equipamento em que foi realizada a espirometria, era iniciado o teste cardiopulmonar com medida direta dos gases expirados, utilizando sensores que permitem a análise respiração a respiração (breath-by-breath), com plotagem em tempo real, da média de sete expirações. Os pacientes respiravam somente através de um adaptador bucal plástico acoplado a pneumotacômetro de diferença de pressão do tipo Pitot ${ }^{67}$, descartável, não valvulado e com $20 \mathrm{ml}$ de espaço morto, tendo sido a cavidade nasal ocluída com grampo. O oxigênio expirado foi medido em célula de zircônio a cerca de $700^{\circ} \mathrm{C}$ por diferença de tensão e o dióxido de carbono em câmara de absorção infravermelha das amostras retiradas por meio de linha de captação direta do pneumotacômetro.

A calibração das medidas de volume do analisador de gases foi feita com uma seringa graduada de 3 litros pertencente ao modelo Cardio $\mathrm{O}_{2}$, 
sendo aceitável erro máximo de $\pm 3 \%$. As condições de temperatura da sala foram controladas e mantidas entre $21^{\circ} \mathrm{C}$ e $23^{\circ} \mathrm{C}$ e registradas a umidade relativa do ar por termo-higrômetro e pressão barométrica em barômetro de Torricelli. O conhecimento e o controle do ambiente local permitiram uma transformação adequada entre ATPS (ambient temperature and pressure, satured), BTPS (body temperature and pressure, dry) e STPD (standard temperature and pressure, dry). Foi realizada calibração dos analisadores de gases usando mistura de gases (oxigênio, dióxido de carbono e nitrogênio) padronizada antes de cada teste, de acordo com a normatização de técnicas e equipamentos para realização de exames em ergometria e ergoespirometria ${ }^{52}$.

Nos testes realizados em bicicleta ergométrica, os pacientes foram orientados a manter constante uma velocidade de pedalagem entre 60 e 80 rotações por minuto, faixa em que a bicicleta utilizada tem o melhor desempenho do mecanismo de compensação de carga. $O$ protocolo utilizado foi o tipo em rampa, com aumento da resistência oferecida ao pedal de mesma intensidade a cada minuto, após aquecimento de dois minutos com carga livre. A fase de recuperação foi ativa, com carga livre nos dois primeiros minutos e passiva nos quatros restantes, com o paciente sentado na bicicleta.

O protocolo do exercício em esteira rolante foi o de Bruce, modificado de forma a torná-lo do tipo em rampa, com incrementos mais suaves a cada dois minutos. A fase de recuperação foi ativa nos dois primeiros minutos, 
com velocidade de $2,7 \mathrm{~km} / \mathrm{h}$, sem inclinação e os quatro restantes com o paciente sentado em cadeira.

O ECG foi continuamente registrado em 12 derivações e a pressão arterial aferida a cada dois minutos, no pico de esforço e na fase de recuperação durante seis a oito minutos com esfigmomanômetro de coluna de mercúrio pelo método auscultatório. Os critérios para interrupção do esforço foram os determinados pelas Diretrizes do Departamento de Ergometria da Sociedade Brasileira de Cardiologia ${ }^{52}$ e os pacientes foram encorajados a manter o esforço até a exaustão. A avaliação subjetiva da intensidade do esforço foi realizada utilizando a Escala Categórica de Borg Modificada 68

A intensidade do esforço foi considerada satisfatória, e o exercício classificado como máximo, se a razão de trocas respiratórias (RER) atingisse valores iguais ou superiores a 1,10 associadas a sintomas de esforço máximo, fadiga ou dispneia.

As seguintes variáveis foram obtidas para análise e expressas em formato tabular ou gráfico: frequência cardíaca $(F C)$ em repouso e no pico do esforço, pressão arterial (PA) em repouso e no pico do esforço, tempo de exercício (total e até o limiar anaeróbico), o consumo de oxigênio $\left(\mathrm{VO}_{2}\right)$ no limiar anaeróbico e pico atingido, produção máxima de dióxido de carbono, ventilação pulmonar (VE), pulso de oxigênio $\left(\mathrm{VO}_{2} / \mathrm{FC}\right)$, equivalentes ventilatórios de $\mathrm{O}_{2}\left(\mathrm{VE} / \mathrm{VO}_{2}\right)$ e $\mathrm{CO}_{2}\left(\mathrm{VE} / \mathrm{VCO}_{2}\right), \mathrm{VE} / \mathrm{VCO}_{2}$ slope, razão de trocas respiratórias - $\mathrm{RER}\left(\mathrm{VCO}_{2} / \mathrm{VO}_{2}\right)$, tempo para que $\circ \mathrm{VO}_{2}$ atingisse metade do seu valor máximo no esforço, na fase de recuperação $\mathrm{VO}_{2}\left(T_{1 / 2}\right)$, 
pressão expiratória final de dióxido de carbono $\left(\mathrm{PETCO}_{2}\right)$ e de oxigênio $\left(\mathrm{PETO}_{2}\right)$, relação espaço morto ventilatório / volume corrente $(\mathrm{Vd} / \mathrm{Vt})$ e a inclinação da curva representativa da relação logarítmica entre a ventilação e o consumo de oxigênio (OUES, Oxygen Uptake Efficiency Slope).

Além das medidas do TCPE realizadas no repouso e no pico do esforço, também foram analisados o comportamento ou as curvas da relação $\mathrm{VO}_{2} /$ carga e do pulso de oxigênio durante o esforço e definidos três tipos de respostas: ascensão normal, platô e queda.

As figuras 5 e 6 mostram um exemplo da curva do pulso de $\mathrm{O}_{2}$ antes e após a cirurgia, em paciente do sexo feminino, 72 anos, submetida ao reparo valvar com sucesso.

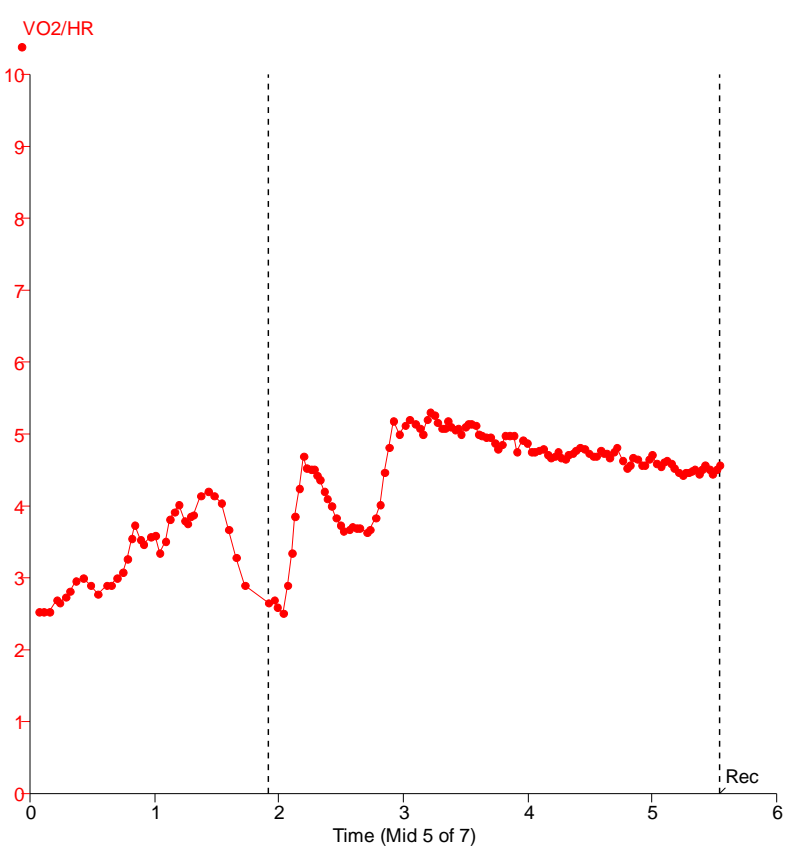

Figura 5 - Curva do pulso de oxigênio pré-operatório. Paciente TU, 72 anos, feminino, submetida ao reparo mitral com sucesso. Observa-se que o pulso de oxigênio passou de 3 para $5 \mathrm{~mL}$.bat ${ }^{-1}$, no início do esforço, decrescendo gradativamente na sequência, indicando redução da performance ventricular esquerda com 0 esforço. As linhas descontínuas indicam o início e o fim do esforço. 


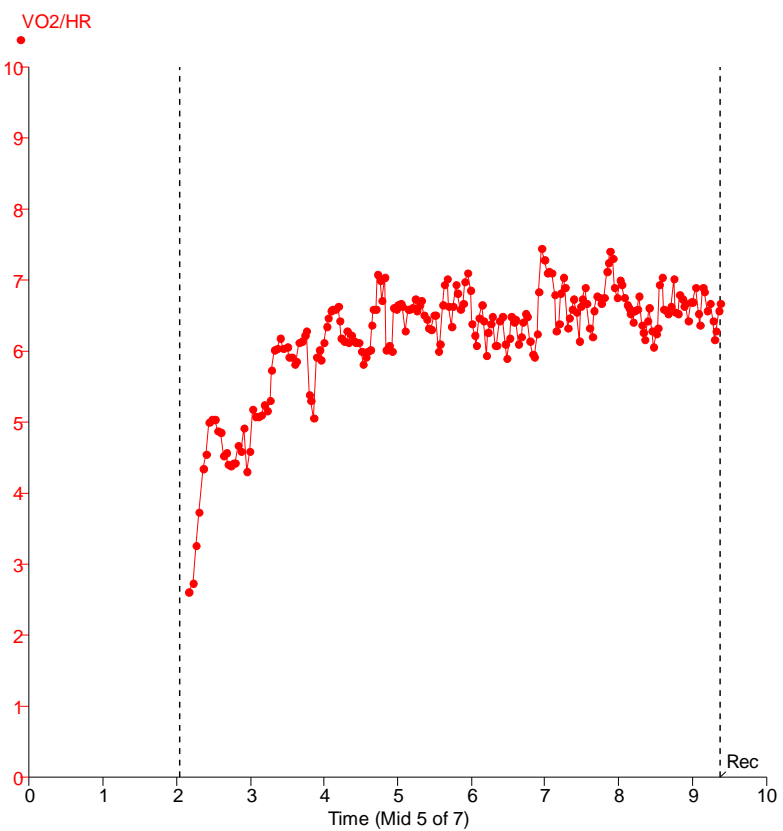

Figura 6 - Curva do pulso de oxigênio pós-operatório. Paciente TU, 72 anos, feminino, submetida ao reparo mitral com sucesso. O pulso de oxigênio passou para $7 \mathrm{~mL}$.bat ${ }^{-1}$ e não mais decresceu com a sequência do exercício. As linhas descontínuas indicam o início e o fim do esforço.

\subsubsection{Cateterismo cardíaco}

O procedimento diagnóstico hemodinâmico foi realizado em todos os pacientes antes da cirurgia para avaliação da função ventricular esquerda e medidas pressóricas. Foi quantificado o volume sistólico e diastólico final do ventrículo esquerdo, com o respectivo cálculo da fração de ejeção (FE).

Para realizar a manometria das câmaras direitas e esquerdas cateterizou-se as mesmas através da via femoral pela técnica de Seldinger e com punção guiada por fluoroscopia na altura da borda inferior da cabeça do fêmur. Colocou-se introdutores 6-French (2 mm de diâmetro) e 7-French (2,3 mm de diâmetro) na artéria e veia femoral direita respectivamente. A seguir, 
avançou-se cateter furo-terminal Lehman 7-French (Medtronic Vascular, Danvers, MA, USA) através do sistema venoso até o $A D, V D$ e artéria pulmonar (AP) e, quando possível, alocou-se o cateter na circulação distal da AP para aferir a pressão de oclusão da mesma (pressão de cunha pulmonar). Com eletrocardiograma (ECG) e curva pressórica simultânea em mm Hg (Polígrafo SP12, TEB, Brasil) recuou-se o cateter registrando as pressões de cunha pulmonar, AP, VD e AD. De forma similar, um cateter pig-tail 6-French (Merit Medical Systems Inc, South Jordan, Utah, USA) foi colocado no ventrículo esquerdo registrando as pressões sistólica e diastólica final (pd2). Posteriormente, sob registro contínuo das curvas de pressão recuou-se o cateter do ventrículo esquerdo para a aorta, com a finalidade de medir a pressão aórtica e detectar a presença de gradiente ventrículo esquerdo/Aorta.

Para a avaliação da IM, realizou-se a ventriculografia esquerda na projeção oblíqua direita $\left(30^{\circ}\right)$, alocando-se a ponta do cateter "pig-tail" na porção medial do VE e injetando-se $25-30 \mathrm{ml}$ de contraste hiposmolar a 20$30 \mathrm{ml}$ por segundo e com $600 \mathrm{~mm} \mathrm{Hg}$ de pressão.

Nos casos de pacientes acima de 35 anos ou com fatores de risco para doença coronariana, foi realizada cinecoronariografia diagnóstica após aferição das pressões das câmaras direitas e esquerdas.

A quantificação das pressões sistólica, diastólica e média foi realizada através de medição direta nas curvas impressas de cunha pulmonar, AP, VD, $A D$, ventrículo esquerdo e aorta. Para a determinação da pressão diastólica final (pd2) do ventrículo esquerdo, usou-se a pressão que coincidiu 
com o início do complexo QRS no registro eletrocardiográfico simultâneo. Nos casos de impossibilidade de aferição direta da pressão de oclusão da AP, usou-se a pressão diastólica da AP como valor substituto.

Em relação à avaliação angiográfica, a análise semiquantitativa da IM foi realizada pela escala de uma a quatro cruzes, conforme a opacificação do átrio esquerdo $(\mathrm{AE})$ durante a ventriculografia esquerda ${ }^{69}$.

A análise quantitativa angiográfica do VE foi realizada com o software XVA versão 7.0 (Medis, Leinden, The Netherlands). Após calibração com a ponta do cateter, o volume diastólico final do ventrículo esquerdo (VDFVE) foi determinado no ponto de maior expansão ventricular, e o volume sistólico final do ventrículo esquerdo (VSFVE) no final do esvaziamento ventricular esquerdo e a partir destes valores foi calculada a FE do ventrículo esquerdo (Figura 7).

As curvas pressóricas e imagens angiográficas foram analisadas por dois operadores experientes no Laboratório de Análises Angiográficas (Corelab) do Instituto Dante Pazzanese de Cardiologia. 

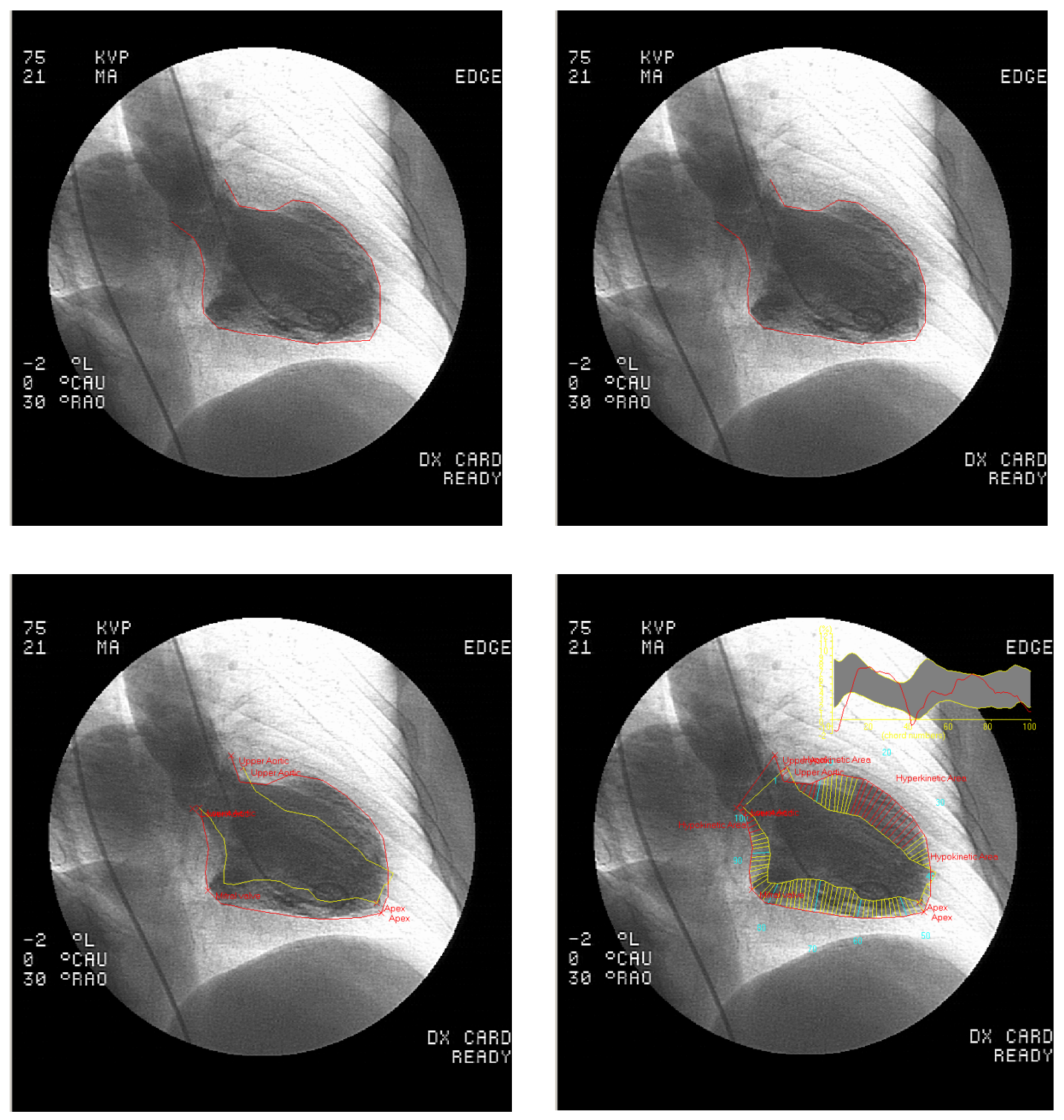

Figura 7 - Estudo hemodinâmico: medidas dos volumes diastólico e sistólico final do ventrículo esquerdo e cálculo da fração de ejeção.

\subsubsection{Método estatístico}

Inicialmente, todas as variáveis foram sujeitas a uma avaliação exploratória, descritiva e gráfica (Box-Plot), com a finalidade de observar o comportamento das medidas e também identificar possíveis erros de 
digitação e casos outliers. Após esta verificação prévia, os resultados foram resumidos em média \pm desvio padrão (DP), mediana e percentis 25 (Per 25) e 75 (Per 75) para variável quantitativa e em freqüências, absolutas (n) e relativas (\%), para variável qualitativa.

A distribuição das medidas quantitativas também foi avaliada pelo teste de normalidade de Kolmogorov-Smirnov ${ }^{70}$. Quando a distribuição normal não foi rejeitada o teste t pareado foi aplicado para a comparação antes e após a cirurgia. Quando a normalidade foi rejeitada ou para medida qualitativa ordinal, o teste não paramétrico de Wilcoxon ou ANOVA não paramétrica para dados ordinais $^{71}$ foi utilizado nesta comparação. A existência de fatores nominais associados às mudanças após a cirurgia foi avaliada pelo teste de McNemar (discordância).

A diferença relativa (\%) entre os resultados pós e pré-operatórios, ou seja, a diferença em relação aos resultados iniciais (pré-operatório), $\Delta$ (\%), foi calculada com o objetivo de observar o comportamento do tamanho destas diferenças após a cirurgia, das medidas ecocardiográficas de remodelamento cardíaco e das variáveis do TCPE. A associação entre estas diferenças relativas do TCPE e ecocardiográficas foi avaliada por correlação de Pearson ou Spearman ${ }^{70}$. Quando as correlações foram significativas, o coeficiente de correlação $r$ foi avaliado. Valores absolutos de $r(|r|)$ sugerem correlação fraca quando $|r|<0,3$, correlação moderada quando $0,3 \leq|r|<0,7$ e alta correlação quando $|r| \geq 0,7$.

A comparação das medidas do TCPE e do ecocardiograma entre os dois grupos de pacientes, o submetido à troca valvar mitral e o 
submetido à cirurgia de reparo mitral, foi feita por teste $t$ de Student ${ }^{72}$ ou não paramétrico de Mann-Whitney ${ }^{70}$ para medida quantitativa. Para as medidas qualitativas a associação com os grupos foi avaliada por teste qui-quadrado de Pearson ou exato de Fisher ${ }^{73}$.

As medidas que apresentaram evidências, mesmo que fracas, entre os grupos, para pelo menos um dos tempos, foram avaliadas por ANOVA para as medidas repetidas ${ }^{74}$, quando a medida quantitativa não rejeitou a distribuição normal ou ANOVA não paramétrica para dados ordinais, quando a medida quantitativa rejeitou distribuição normal ${ }^{71}$, com o objetivo de verificar o efeito da interação entre tempo e grupo, que quando significativo pode indicar que o comportamento de um grupo no período após a cirurgia foi diferente do outro.

Os resultados cujas diferenças apresentaram nível descritivo (valor de p) dos testes inferiores a 0,05 foram considerados estatisticamente significantes. Os casos em que $5 \% \leq$ valor de $p<10 \%$ sugeriram fracas evidências de diferenças significativas.

Todos os cálculos estatísticos foram realizados utilizando programa estatístico SPSS for Windows, versão 19, Chicago, IL, USA. 
Resultados 


\subsection{Características da população}

A população estudada compreendeu 78 indivíduos portadores de IM crônica, orgânica e não isquêmica, com indicação cirúrgica para correção da valvopatia. Foram excluídos seis pacientes que não completaram o protocolo por complicações pós-operatórias, sendo quatro pacientes que evoluíram para óbito no pós-operatório hospitalar, um paciente que apresentou acidente vascular cerebral com sequela motora em dimídio esquerdo e uma paciente que foi submetida à amputação da perna direita após evento tromboembólico no pós-operatório.

Entre agosto de 2008 e janeiro de 2011, 72 pacientes (média das idades de 49,89 $\pm 16,94$ anos) e sexo masculino em 41 pacientes (56,9\%) foram consecutivamente e prospectivamente avaliados. A etiologia predominante foi a degenerativa (50 pacientes - 69,4\%), 51 pacientes (70,8\%) estavam em classe funcional I ou II pela NYHA e oito pacientes $(11,1 \%)$ apresentavam FA permanente. As principais características clínicas pré-operatórias, expressas em frequência (n e \%), estão apresentadas na tabela 1. 
Tabela 1 - Características clínicas pré-operatórias dos 72 indivíduos portadores de IM grave e incluídos no protocolo

\begin{tabular}{lcc}
\hline Características Clínicas & $\mathbf{n = 7 2}$ & $\%$ \\
\hline Sexo M/F & $41 / 31$ & $56,9 / 43,1$ \\
Etiologia & & \\
PVM & 50 & 69,4 \\
Reumática & 20 & 27,8 \\
Outras & 2 & 2,8
\end{tabular}

\section{NYHA classe funcional}
I / II
$19 / 32$
$26,4 / 44,4$
III / IV
$19 / 2$
$26,4 / 2,8$

ECG

RS / FA

64 / 8

SAE

61

$88,9 / 11,1$

SVE

14

95,3

BRD

2

19,4

BRE

0

2,8

Hipertensão arterial sistêmica

39

0

Diabetes

6

54,2

Obesidade

14

8,3

Fumante ativo

6

19,4

Dislipidemia

15

8,3

Medicamentos em uso:

Diurético

32

20,8

Betabloqueador

31

IECA

36

44,4

BRA

5

Amiodarona

4

43,1

50,0

6,9

5,6

PVM, prolapso da valva mitral; NYHA, New York Heart Association; RS, ritmo sinusal; FA, fibrilação atrial; SAE, sobrecarga atrial esquerda; SVE, sobrecarga ventricular esquerda; $\mathrm{BRD}$, bloqueio de ramo direito; BRE, bloqueio de ramo esquerdo; IECA, inibidor da enzima conversora da angiotensina; AT1, angiotensina 1; BRA, bloqueador do receptor da angiotensina 1. 


\subsection{Cirurgia da valva mitral: escolha da prótese, alterações anatômicas do aparato mitral e técnicas de reparo}

A decisão sobre o tipo de cirurgia (reparo ou troca valvar) ficou a critério do cirurgião e baseada nos dados (clínicos e ecocardiográficos) préoperatórios e na avaliação anatômica do aparato mitral no intraoperatório. $\mathrm{O}$ tipo de prótese (bioprótese ou mecânica) foi definido pelo cardiologista assistente após análise individual de características clínicas e na condição adequada de anticoagulação permanente.

O reparo mitral foi a cirurgia mais frequentemente realizada (47 pacientes-65,3\%) e 25 pacientes foram submetidos à troca valvar por bioprótese (19 pacientes-26,4\%) ou prótese mecânica de duplo-disco (seis pacientes-8,3\%). A preservação das estruturas subvalvares associadas à troca valvar foi realizada em 17 pacientes (68\%). Dos 72 pacientes, três $(4,2 \%)$ realizaram plastia da valva tricúspide com retalho de pericárdio bovino. Durante o ato operatório, o tempo médio de clampeamento da aorta foi de 50,1 \pm 16,2 $\min$ e 0 tempo de circulação extracorpórea de $69,6 \pm 19,9$ minutos.

As alterações anatômicas do aparato mitral durante a cirurgia nos 72 pacientes incluídos no estudo e as técnicas cirúrgicas realizadas nos 47 pacientes submetidos ao reparo mitral estão discriminadas nas tabelas 2 e 3 . 
Tabela 2 -Cirurgia: descrição das alterações anatômicas do aparato valvar mitral nos 72 pacientes incluídos no estudo

\begin{tabular}{|c|c|c|}
\hline Alterações anatômicas na cirurgia & $\mathrm{n}=72$ & $\%$ \\
\hline Dilatação do anel mitral & 70 & 97,2 \\
\hline $\begin{array}{l}\text { Ruptura de cordas tendíneas da cúspide } \\
\text { posterior }\end{array}$ & 27 & 37,5 \\
\hline Retração fibrótica das cúspides & 27 & 37,5 \\
\hline $\begin{array}{l}\text { Alongamento de cordas tendíneas da cúspide } \\
\text { anterior }\end{array}$ & 24 & 33,3 \\
\hline $\begin{array}{l}\text { Alongamento de cordas tendíneas da cúspide } \\
\text { posterior }\end{array}$ & 15 & 20,8 \\
\hline Megafolheto posterior & 11 & 15,3 \\
\hline Ruptura de cordas tendíneas da cúspide anterior & 8 & 11,1 \\
\hline Subcomissura & 8 & 11,1 \\
\hline Fusão comissural & 4 & 5,6 \\
\hline Fusão de cordas tendíneas & 4 & 5,6 \\
\hline Fenda na cúspide anterior & 3 & 4,2 \\
\hline
\end{tabular}


Tabela 3 - Reparo mitral: descrição das técnicas cirúrgicas realizadas nos 47 pacientes do estudo submetidos ao reparo mitral

\begin{tabular}{|c|c|c|}
\hline Técnicas cirúrgicas & $\mathrm{n}=47$ & $\%$ \\
\hline Anuloplastia do anel mitral posterior & 47 & 100,0 \\
\hline Ressecção quadrangular da cúspide posterior & 30 & 63,8 \\
\hline Ressecção parcial da cúspide anterior & 6 & 12,8 \\
\hline $\begin{array}{l}\text { Encurtamento de cordas tendíneas da cúspide } \\
\text { anterior }\end{array}$ & 5 & 10,6 \\
\hline Fechamento de fenda da cúspide anterior & 2 & 4,3 \\
\hline $\begin{array}{l}\text { Transferência de cordas tendíneas da cúspide } \\
\text { posterior para a anterior }\end{array}$ & 2 & 4,3 \\
\hline $\begin{array}{l}\text { Aumento da cúspide posterior com retalho de } \\
\text { pericárdio bovino }\end{array}$ & 1 & 2,1 \\
\hline Neocorda do folheto posterior & 1 & 2,1 \\
\hline Comissurotomia mitral & 1 & 1,4 \\
\hline Cordotomia anterior e posterior & 1 & 4,5 \\
\hline
\end{tabular}

\subsection{Cirurgia: resultados imediatos}

Houve quatro óbitos $(5,1 \%)$ no pós-operatório imediato (fase hospitalar), sendo três $(3,9 \%)$ em decorrência de choque séptico no $63^{\circ}$ dia, no $52^{\circ}$ dia e no $12^{\circ}$ dia de pós-operatório, respectivamente e um $(1,3 \%)$ por choque cardiogênico no pós-operatório imediato. 
As principais complicações pós-operatórias na fase hospitalar foram: FA aguda em 23 pacientes $(31,9 \%)$, bloqueio atrioventricular total com reversão espontânea em dois pacientes $(2,8 \%)$, pneumonia nosocomial em dois pacientes $(2,8 \%)$, e um paciente $(1,4 \%)$ evoluiu no pós-operatório com insuficiência mitral residual importante e realizado a troca valvar por bioprótese no $8^{\circ}$ dia de pós-operatório. $\mathrm{O}$ achado cirúrgico desse paciente revelou insuficiência mitral por laceração da cúspide posterior junto ao anel mitral.

Os pacientes receberam alta hospitalar em média 10,2 $\pm 9,8$ dias (mediana $=7,0$ dias) após a cirurgia.

\subsection{Variáveis clínicas e laboratoriais pré e pós-operatório}

Os exames laboratoriais foram realizados em média $22,4 \pm 22,8$ dias antes da cirurgia e 212,7 $\pm 41,6$ dias após a cirurgia.

$\mathrm{Na}$ avaliação comparativa das medidas antropométricas realizadas no dia do TCPE, houve um significativo aumento pós-operatório do peso e índice de massa corporal (IMC): peso de 70,67 $\pm 17,07 \mathrm{Kg}$ para 72,09 \pm $17,16 \mathrm{Kg}(\mathrm{p}=0,016)$ e IMC de 25,44 $\pm 5,01$ para 25,97 $\pm 5,12(\mathrm{p}=0,011)$.

A classe funcional (CF) pela NHYA melhorou significativamente após a cirurgia de em média 2,06 $\pm 0,80$ para $1,17 \pm 0,38(p<0,001)$.

A presença de $F A$, em oito pacientes $(11,1 \%)$ antes da cirurgia reduziu-se para seis pacientes $(8,3 \%)$ no pós-operatório $(p=N S)$. 
Com relação às medicações utilizadas na vigência da realização dos TCPE pré e pós-operatório, pode-se observar um maior número de pacientes em uso de betabloqueador no pós-operatório (30 para 38 pacientes, $\mathrm{p}=\mathrm{NS}$ ) e uma maior proporção de pacientes no momento pósoperatório recebendo medicação bloqueadora dos receptores da angiotensina AT1 (de 7,2\% para 15,7\%, p=0,052) e amiodarona (de 5,8\% para $20,0 \%, p=0,014)$. Não houve diferença significativa na proporção de pacientes que receberam terapia diurética ou inibidores da enzima conversora da angiotensina, no período pré e pós-operatório.

As seguintes variáveis clínicas e laboratoriais pré e pós-operatórias foram analisadas, comparadas e expressas em frequência ( $\mathrm{n}$ e \%), ou média \pm DP e mediana (Tabela 4). 
Tabela 4 - Características de base: quadro comparativo pré e pósoperatório

\begin{tabular}{|c|c|c|c|}
\hline $\begin{array}{l}\text { Características } \\
\text { Clínicas e Laboratoriais }\end{array}$ & PRÉ-OP. & PÓS-OP. & $\mathbf{p}$ \\
\hline Peso, Kg & $\begin{array}{c}70,67 \pm 17,07 \\
68,00\end{array}$ & $\begin{array}{c}72,09 \pm 17,16 \\
71,00\end{array}$ & $0,016^{t}$ \\
\hline ASC, $\mathrm{m}^{2}$ & $\begin{array}{c}1,79 \pm 0,25 \\
1,79\end{array}$ & $\begin{array}{c}1,79 \pm 0,32 \\
1,80\end{array}$ & $0,710^{t}$ \\
\hline IMC, kg./m² & $\begin{array}{l}25,44 \pm 5,01 \\
24,33\end{array}$ & $\begin{array}{l}25,97 \pm 5,12 \\
25,20\end{array}$ & $0,011^{t}$ \\
\hline CF & $\begin{array}{l}2,06 \pm 0,80 \\
2,00\end{array}$ & $\begin{array}{c}1,17 \pm 0,38 \\
1,00\end{array}$ & $<0,001^{\mathrm{W}}$ \\
\hline FA & $8(11,1 \%)$ & $6(8,3 \%)$ & 0,697 \\
\hline Diurético & $32(44,4 \%)$ & $27(38,6 \%)$ & 0,202 \\
\hline IECA & $36(52,2 \%)$ & $34(48,6 \%)$ & 0,578 \\
\hline BRA & $5(7,2 \%)$ & $11(15,7 \%)$ & 0,052 \\
\hline Betabloqueador & $30(43,5 \%)$ & $38(54,3 \%)$ & 0,112 \\
\hline Amiodarona & $4(5,8 \%)$ & $14(20,0 \%)$ & 0,014 \\
\hline Glicemia, mg.dL ${ }^{-1}$ & $\begin{array}{c}86,8 \pm 13,7 \\
86,0\end{array}$ & $\begin{array}{c}90,2 \pm 14,2 \\
90,5\end{array}$ & $0,029^{t}$ \\
\hline Ureia, mg.dL ${ }^{-1}$ & $\begin{array}{c}36,6 \pm 10,2 \\
36,0\end{array}$ & $\begin{array}{c}34,4 \pm 9,7 \\
33,0\end{array}$ & $0,028^{t}$ \\
\hline Creatinina, $\mathrm{mg} \mathrm{dL}^{-1}$ & $\begin{array}{c}0,96 \pm 0,27 \\
0,90\end{array}$ & $\begin{array}{c}1,00 \pm 0,25 \\
1,00\end{array}$ & $0,023^{\mathrm{W}}$ \\
\hline Hemoglobina, g.dL ${ }^{-1}$ & $\begin{array}{c}14,2 \pm 1,5 \\
14,00\end{array}$ & $\begin{array}{c}13,9 \pm 1,7 \\
13,80\end{array}$ & $0,023^{t}$ \\
\hline Hematócrito, \% & $\begin{array}{l}42,5 \pm 4,2 \\
42,2\end{array}$ & $\begin{array}{c}42,1 \pm 4,7 \\
41,6\end{array}$ & $0,315^{t}$ \\
\hline
\end{tabular}

ASC, área de superfície corpórea; IMC, índice de massa corporal; $C F$, classe funcional pela NYHA; FA, fibrilação atrial, IECA, inibidor da enzima de conversão da angiotensina; BRA, bloqueador do receptor da angiotensina AT1; K, teste Kappa; t, teste t-pareado; W, teste de Wilcoxon 


\subsection{Ecocardiograma pré e pós-operatório}

Os estudos ecocardiográficos pré e pós-operatórios foram realizados em média 42,2 \pm 47,8 dias (mediana $=22,5$ dias) antes e 218,1 $\pm 37,2$ dias (mediana = 203,0 dias) após a cirurgia.

O estudo ecocardiográfico pré-operatório revelou as seguintes médias das medidas de gravidade da IM: vena contracta de 0,76 $\pm 0,11 \mathrm{~cm}$, área do orifício efetivo de refluxo de $0,67 \pm 0,24 \mathrm{~cm}^{2}$ e volume de regurgitação de $109,10 \pm 36,22 \mathrm{ml}$.

No ecocardiograma pré-operatório, quatro pacientes $(5,6 \%)$ apresentavam insuficiência tricúspide (IT) de grau moderado, um paciente $(1,4 \%)$ IT grave e 50 pacientes $(69,4 \%)$ com algum grau de hipertensão arterial pulmonar (PSAP > $35 \mathrm{~mm} \mathrm{Hg}$ ). No estudo pós-operatório, oito pacientes $(11,1 \%)$ apresentavam IT moderada, nenhum com IT grave e 33 pacientes $(45,8 \%)$ evoluíram com HAP.

Todos os pacientes que realizaram troca valvar apresentaram próteses normofuncionantes e dos 47 pacientes que realizaram o reparo valvar, 33 pacientes $(70,2 \%)$ evoluíram com refluxo residual ausente, mínimo ou discreto, três pacientes $(6,4 \%)$ com refluxo residual discreto a moderado, sete pacientes $(14,9 \%)$ com refluxo residual moderado, dois pacientes $(4,3 \%)$ com refluxo residual moderado a importante e dois pacientes $(4,3 \%)$ com refluxo residual importante. Os dois pacientes com IM residual importante não realizaram o TCPE pós-operatório por terem sido reoperados 
antes e foram excluídos do banco de dados do TCPE. Os demais pacientes com IM residual estão em acompanhamento clínico.

Os principais resultados do estudo ecocardiográfico foram comparados antes e após a cirurgia e estão discriminados na tabela 5 .

Após a cirurgia houve significativas reduções das seguintes medidas ecocardiográficas: volume do átrio esquerdo (VAE) de 124,4 \pm 58,9 ml para $76,4 \pm 34,0 \mathrm{ml}(\mathrm{p}<0,0001), \mathrm{VDFVE}$ de $151,7 \pm 35,8 \mathrm{~mL}$ para $109,6 \pm 42,8 \mathrm{~mL}$ ( $p<0,0001)$, VSFVE de $47,6 \pm 18,0 \mathrm{~mL}$ para $43,9 \pm 27,1 \mathrm{~mL}(p<0,001)$, FE de $69,1 \pm 6,4 \%$ para $61,3 \pm 8,2 \%(p<0,0001)$ e pressão sistólica da artéria pulmonar (PSAP) de 46,7 $\pm 15,9 \mathrm{~mm} \mathrm{Hg}$ para $35,4 \pm 12,5 \mathrm{~mm} \mathrm{Hg}$ $(p<0,0001)$. A comparação das médias e a diferença relativa das principais medidas ecocardiográficas pré e pós-operatória estão inseridas na tabela 5. 
Tabela 5 - Comparação dos parâmetros ecocardiográficos de remodelamento cardíaco no pré e pós-operatórios

\begin{tabular}{|c|c|c|c|}
\hline \multirow[t]{2}{*}{ Ecocardiograma } & \multicolumn{2}{|c|}{$\begin{array}{c}\text { MÉDIA } \pm \text { DP } \\
\text { MFDIANA (Per25:Per75) }\end{array}$} & \multirow[t]{2}{*}{$\mathbf{p}$} \\
\hline & Pré-op. & Pós-op. & \\
\hline VAE, $\mathrm{mm}^{3}$ & $\begin{array}{c}124,4 \pm 58,9 \\
114,5(90,5 ; 142,0)\end{array}$ & $\begin{array}{c}76,4 \pm 34,0 \\
68,0(50,0 ; 95,8)\end{array}$ & $<0,001^{\mathrm{t}}$ \\
\hline$\Delta \mathrm{VAE}, \%$ & \multicolumn{2}{|c|}{$-36,0 \pm 19,7$} & \\
\hline DDFVE, mm & $\begin{array}{c}61,6 \pm 4,8 \\
61,5(58,0 ; 64,0)\end{array}$ & $\begin{array}{c}52,2 \pm 6,0 \\
52,0(48,0 ; 55,5)\end{array}$ & $<0,001^{t}$ \\
\hline$\Delta$ DDFVE, $\%$ & \multicolumn{2}{|c|}{$\begin{array}{c}-15,0 \pm 8,0 \\
-15,5(-20,5 ;-9,4)\end{array}$} & \\
\hline DSFVE, mm & $\begin{array}{c}38,8 \pm 4,4 \\
38,0(36,0 ; 40,0)\end{array}$ & $\begin{array}{c}35,1 \pm 6,3 \\
35,0(31,5 ; 37,5)\end{array}$ & $<0,001^{n}$ \\
\hline$\Delta$ DSFVE, $\%$ & \multicolumn{2}{|c|}{$\begin{array}{c}-9,2 \pm 12,0 \\
-10,8(-17,1 ;-2,7)\end{array}$} & \\
\hline VDFVE, mL & $\begin{array}{c}151,7 \pm 35,8 \\
152,0(130,0 \\
173,0)\end{array}$ & $\begin{array}{c}109,6 \pm 42,8 \\
101,0(84,0 ; 124,5)\end{array}$ & $<0,001^{n}$ \\
\hline$\Delta$ VDFVE, $\%$ & \multicolumn{2}{|c|}{$-27,4 \pm 20,1$} & \\
\hline VSFVE, mL & $\begin{array}{c}47,6 \pm 18,0 \\
44,0(36,0 ; 56,0)\end{array}$ & $\begin{array}{c}43,9 \pm 27,1 \\
38,0(30,0 ; 49,5)\end{array}$ & $0,001^{\mathrm{W}}$ \\
\hline$\Delta$ VSFVE, $\%$ & \multicolumn{2}{|c|}{$-7,1 \pm 32,1$} & \\
\hline FE, \% & $\begin{array}{c}69,1 \pm 6,4 \\
70,0(66,0 ; 74,0)\end{array}$ & $\begin{array}{c}61,3 \pm 8,2 \\
63,0(58,0 ; 66,0)\end{array}$ & $<0,001^{\mathrm{t}}$ \\
\hline$\Delta \mathrm{FE}, \%$ & \multicolumn{2}{|c|}{$-11,0 \pm 11,7$} & \\
\hline PSAP, mm Hg & $\begin{array}{c}46,7 \pm 15,9 \\
42,0(36,0 ; 50,5)\end{array}$ & $\begin{array}{c}35,4 \pm 12,5 \\
36,0(31,0 ; 40,0)\end{array}$ & $<0,001^{n}$ \\
\hline$\Delta$ PSAP, $\%$ & \multicolumn{2}{|c|}{$\begin{array}{c}-22,6 \pm 28,5 \\
-19,3(-36,1 ;-5,4)\end{array}$} & \\
\hline
\end{tabular}

VAE, volume do átrio esquerdo; DDFVE, diâmetro diastólico final do VE; DSFVE, diâmetro sistólico final do VE; VDFVE, volume diastólico final do VE; VSFVE, volume sistólico final do VE; FE, fração de ejeção; $\Delta$, variação percentual; PSAP, pressão sistólica da artéria pulmonar; $\Delta$, diferença relativa; $\mathrm{t}$, teste $\mathrm{t}$-pareado; $\mathrm{W}$, teste de Wilcoxon 


\subsection{Estudo hemodinâmico pré-operatório e comparação com as medidas do ecocardiograma}

Os estudos hemodinâmicos foram realizados em média $81,0 \pm 110,4$ dias (mediana $=38,0$ dias) antes da cirurgia e as medidas pressóricas, volume diastólico e sistólico final do VE e fração de ejeção (FE) estão discriminados na tabela 6.

Tabela 6 - Estudo hemodinâmico pré-operatório

\begin{tabular}{lcc}
\hline CATE & MÉDIA (DP) & MEDIANA (Per 25; Per 75) \\
\hline PCP, $\mathbf{m m H g}$ & $19,6(11,7)$ & $17,5(12,0 ; 25,0)$ \\
PDVE, $\mathbf{m m H g}$ & $15,9(11,1)$ & $14,5(10,0 ; 20,0)$ \\
ADm, $\mathbf{m m H g}$ & $7,4(4,6)$ & $7,0(3,0 ; 10,0)$ \\
PSAP, $\mathbf{m m H g}$ & $39,0(13,7)$ & $37,0(27,0 ; 47,0)$ \\
VDFVE, $\mathbf{m L}$ & $187,0(67,9)$ & $168,0(146,1 ; 218,1)$ \\
VSFVE, $\mathbf{m L}$ & $61,4(39,7)$ & $52,6(34,1 ; 74,9)$ \\
FE, \% & $68,3(11,9)$ & $70,2(61,8 ; 76,9)$
\end{tabular}

$\mathrm{PCP}$, pressão capilar pulmonar; PDVE, pressão diastólica final do VE; ADm, pressão média em átrio direito; PSAP, pressão sistólica em artéria pulmonar; VDFVE, volume diastólico final do VE; VSFVE, volume sistólico final do VE; FE, fração de ejeção

As medidas pré-operatórias de função ventricular esquerda e da PSAP realizadas pelo cateterismo cardíaco foram comparadas com as medidas do ecocardiograma pré-operatório (tabela 7). Em média, as medidas do VDFVE medido pelo CATE foram maiores do que o medido pelo ECO, assim como o VSFVE e a PSAP. A FE foi ligeiramente menor na avaliação pelo CATE. 
Tabela 7 - Comparação pré-operatória entre as medidas do ecocardiograma e estudo hemodinâmico

\begin{tabular}{|c|c|c|c|}
\hline & $\begin{array}{c}\text { ECO } \\
\text { Média (DP) } \\
\text { Mediana }\end{array}$ & $\begin{array}{c}\text { CATE } \\
\text { Média (DP) } \\
\text { Mediana }\end{array}$ & $\mathbf{p}$ \\
\hline VDFVE, mL & $151,7(35,8)$ & $187,0(67,9)$ & $0,460^{t}$ \\
\hline VSFVE, mL & $\begin{array}{c}152,0 \\
47,6(18,0)\end{array}$ & $\begin{array}{c}168,0 \\
61,4(39,7)\end{array}$ & $0,054^{\mathrm{W}}$ \\
\hline FE, \% & $\begin{array}{c}44,0 \\
69,1(6,4)\end{array}$ & $\begin{array}{c}52,6 \\
68,3(11,9)\end{array}$ & $0,075^{t}$ \\
\hline PSAP, mmHg & $\begin{array}{c}70,0 \\
46,7(15,9)\end{array}$ & $\begin{array}{c}70,2 \\
39,0(13,7)\end{array}$ & $0,004^{\mathrm{W}}$ \\
\hline 2ГIГ & 42,0 & 37,0 & 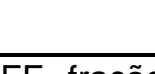 \\
\hline
\end{tabular}

\subsection{Prova de função pulmonar pré e pós-operatório}

Nossos resultados evidenciaram uma leve redução após a cirurgia da capacidade vital forçada (CVF) e volume expiratório forçado no primeiro segundo (VEF1): CVF de 3,52 $\pm 0,90$ litros para $3,39 \pm 0,94$ litros $(p=0,374)$ e VEF1 de 2,76 \pm 0,77 litros para 2,73 $\pm 0,79$ litros $(p=0,317)$, ambos sem significância estatística. Após a cirurgia houve um aumento significativo da ventilação voluntária máxima (VVM) de 108,55 \pm 29,19 L.min-1 para $111,17 \pm 33,13$ L.min-1 $(p=0,051)$. A tabela 8 mostra os principais resultados das provas de função pulmonar realizadas nos momentos pré e pósoperatório. 
Tabela 8 - Prova de Função Pulmonar: comparação entre as medidas pré e pós-operatórias

\begin{tabular}{|c|c|c|c|}
\hline $\begin{array}{l}\text { PROVA DE FUNÇÃO } \\
\text { PULMONAR }\end{array}$ & $\begin{array}{c}\text { PRÉ-OP } \\
\text { Média (DP) } \\
\text { Mediana } \\
\end{array}$ & $\begin{array}{c}\text { PÓS-OP } \\
\text { Média (DP) } \\
\text { Mediana } \\
\end{array}$ & $\mathbf{p}$ \\
\hline CVF, L & $\begin{array}{c}3,52(0,90) \\
3,57\end{array}$ & $\begin{array}{c}3,39(0,94) \\
3,33\end{array}$ & $0,374^{t}$ \\
\hline CVF (\% predito) & $\begin{array}{c}95,42(15,14) \\
95,30\end{array}$ & $\begin{array}{c}95,02(15,98) \\
95,10\end{array}$ & $0,730^{t}$ \\
\hline VEF1, L & $\begin{array}{c}2,76(0,77) \\
2,80\end{array}$ & $\begin{array}{c}2,73(0,79) \\
2,65\end{array}$ & $0,317^{t}$ \\
\hline VEF1 (\% predito) & $\begin{array}{c}90,80(15,84) \\
91,60\end{array}$ & $\begin{array}{c}93,68(17,96) \\
93,80\end{array}$ & $0,195^{t}$ \\
\hline VEF1/CVF & $\begin{array}{c}80,95(10,59) \\
80,00\end{array}$ & $\begin{array}{c}81,19(7,27) \\
82,00\end{array}$ & $0,785^{t}$ \\
\hline VVM, L.min-1 & $\begin{array}{c}108,55(29,19) \\
110,20\end{array}$ & $\begin{array}{c}111,17(33,13) \\
112,50\end{array}$ & $0,051^{t}$ \\
\hline VVM (\% predito) & $\begin{array}{c}91,05(21,20) \\
90,45\end{array}$ & $\begin{array}{c}94,80(24,84) \\
94,50\end{array}$ & $0,029^{t}$ \\
\hline
\end{tabular}

\subsection{Teste cardiopulmonar de exercício pré e pós-operatórios}

As variáveis do TCPE realizado na bicicleta e na esteira, realizados antes e após a cirurgia de correção da valvopatia, foram analisadas e comparadas. Os resultados estão descritos a seguir. 


\subsubsection{Efeito da cirurgia nas variáveis do teste cardiopulmonar de exercício realizado na bicicleta}

Os TCPE na bicicleta ergométrica pré e pós-operatórios foram realizados em média 23,7 $\pm 27,9$ dias (mediana $=15,0$ dias) antes e 229,6 \pm 54,8 dias (mediana= 207,0 dias) após a cirurgia, respectivamente.

Após a correção cirúrgica da IM houve diminuição do $\mathrm{VO}_{2}$ pico de $1356,0 \pm 506,2 \mathrm{~mL} \cdot \mathrm{min}^{-1}$ para 1269,0 \pm 408,0 mL.min ${ }^{-1}(\mathrm{p}=0,015)$ e do $\mathrm{VO}_{2}$ no limiar anaeróbio de 775,7 $\pm 267,5 \mathrm{~mL} \cdot \mathrm{min}^{-1}$ para 707,6 \pm 266,3 mL. $\mathrm{min}^{-1}$ $(p=0,042)$. O equivalente metabólico (MET) no pico do esforço diminuiu de $5,5 \pm 1,7 \mathrm{~mL} \cdot \mathrm{kg}^{-1} \cdot \mathrm{min}^{-1}$ no pré-operatório para $5,1 \pm 1,3 \mathrm{~mL} \cdot \mathrm{kg}^{-1} \cdot \mathrm{min}^{-1}$ no pósoperatório. Após a cirurgia, a FC pico diminuiu de 144,3 $\pm 27,2$ bpm para $126,5 \pm 24,6 \mathrm{bpm}(\mathrm{p}<0,0001)$. O pulso de $\mathrm{O}_{2}$ aumentou após a cirurgia de $9,5 \pm 3,4 \mathrm{~mL}$ bat $^{-1}$ para 10,1 $\pm 3,1 \mathrm{~mL}$ bat $^{-1}\left(p=0,09^{\mathrm{W}}\right)$. A variável VE $/ \mathrm{VCO}_{2}$ slope apresentou leve redução após a cirurgia de 34,94 \pm 9,95 para $32,42 \pm 5,40(p=0,078)$ e a variável OUES apresentou significativa redução após a cirurgia de $1530,0 \pm 516,3$ no pré-operatório para $1413,8 \pm 414,6$ no pós-operatório $(p=0,001)$. A reserva ventilatória aumentou após a cirurgia de $48,4 \pm 15,9 \%$ para $53,3 \pm 13,5 \% \quad(p=0,082)$. Não houve alterações significativas na duração do tempo para que $\circ \mathrm{VO}_{2}$ atingisse metade do seu valor máximo do esforço na fase de recuperação (T1/2) após a cirurgia.

A tabela 9 mostra as comparações pré e pós-operatórias das variáveis analisadas do TCPE realizados na bicicleta, assim como a variação 
percentual das diferenças do $\mathrm{VO}_{2}$ pico, pulso $\mathrm{O}_{2}$ pico, $\mathrm{T}_{1 / 2}, \mathrm{VE} / \mathrm{VCO}_{2}$ slope e OUES.

Tabela 9 - Efeito da cirurgia nas variáveis dos TCPE realizados na bicicleta

\begin{tabular}{|c|c|c|c|}
\hline $\begin{array}{l}\text { TCPE } \\
\text { BICICLETA }\end{array}$ & $\begin{array}{c}\text { PRÉ-OP } \\
\text { Média (DP) } \\
\text { Mediana (Per25; Per75) }\end{array}$ & $\begin{array}{c}\text { PÓS-OP } \\
\text { Média (DP) } \\
\text { Mediana (Per25; Per75) }\end{array}$ & $\mathbf{p}$ \\
\hline Tempo de exercício, min. & $\begin{array}{c}10,2(4,1) \\
10,9(8,3 ; 12,5)\end{array}$ & $\begin{array}{c}11,2(3,6) \\
11,0(8,9 ; 13,3)\end{array}$ & $0,112^{t}$ \\
\hline FC repouso, bpm & $\begin{array}{c}80,4(15,9) \\
77,0(68,5 ; 90,5)\end{array}$ & $\begin{array}{c}82,8(14,3) \\
82,0(72,0 ; 92,3)\end{array}$ & $0,240^{t}$ \\
\hline FC pico, bpm & $\begin{array}{c}144,3(27,2) \\
147,0(121,0 ; 162,0)\end{array}$ & $\begin{array}{c}126,5(24,6) \\
127,0(110,0 ; 142,0)\end{array}$ & $<0,001^{\dagger}$ \\
\hline FC max predita, bpm & $\begin{array}{c}171,0(16,7) \\
168,0(159,0 ; 185,0)\end{array}$ & $\begin{array}{c}170,2(16,7) \\
168,0(156,5 ; 184,5)\end{array}$ & \\
\hline RER pico & $\begin{array}{c}1,15(0,13) \\
1,16(1,07 ; 1,22)\end{array}$ & $\begin{array}{c}1,19(0,14) \\
1,20(1,11 ; 1,28)\end{array}$ & $0,036^{t}$ \\
\hline MET pico, mL.kg ${ }^{-1} \cdot \mathrm{min}^{-1}$ & $\begin{array}{c}5,5(1,7) \\
5,5(4,7 ; 6,3)\end{array}$ & $\begin{array}{c}5,1(1,3) \\
4,9(4,3 ; 6,0)\end{array}$ & $0,009^{t}$ \\
\hline $\begin{array}{l}\text { MET predito, mL.kg } \\
{ }^{1} \cdot \mathrm{min}^{-1}\end{array}$ & $\begin{array}{c}8,6(2,0) \\
8,3(7,1 ; 9,9)\end{array}$ & $\begin{array}{c}8,4(2,1) \\
8,2(6,9 ; 9,6)\end{array}$ & \\
\hline Carga pico, Watts & $\begin{array}{c}86,3(42,9) \\
90,0(50,0 ; 110,0)\end{array}$ & $\begin{array}{c}89,1(39,2) \\
90,0(64,5 ; 113,5)\end{array}$ & $0,434^{t}$ \\
\hline $\mathrm{VO}_{2}(\mathrm{LA}), \mathrm{mL} \cdot \mathrm{min}^{-1}$ & $\begin{array}{c}776(268) \\
720(599 ; 911)\end{array}$ & $\begin{array}{c}708(266) \\
695(504 ; 803)\end{array}$ & $0,042^{t}$ \\
\hline $\mathrm{VO}_{2}$ pico, mL.min ${ }^{-1}$ & $\begin{array}{c}1356(506) \\
1277(983 ; 1678)\end{array}$ & $\begin{array}{c}1269(408) \\
1244(943 ; 1507)\end{array}$ & $0,015^{t}$ \\
\hline$\Delta \mathrm{VO}_{2}$ pico, \% & $\begin{array}{r}-3,0 \\
-6,9(-17\end{array}$ & $\begin{array}{l}0,2) \\
3 ; 7,4)\end{array}$ & \\
\hline $\mathrm{VO}_{2}(\mathrm{LA}), \mathrm{mL} \cdot \mathrm{kg}^{-1} \cdot \mathrm{min}^{-1}$ & $\begin{array}{c}11,1(2,9) \\
10,9(9,2 ; 13,2)\end{array}$ & $\begin{array}{c}9,8(3,0) \\
9,2(7,5 ; 11,5)\end{array}$ & $0,005^{t}$ \\
\hline $\mathrm{VO}_{2}$ pico, $\mathrm{mL} \cdot \mathrm{kg}^{-1} \cdot \mathrm{min}^{-1}$ & $\begin{array}{c}19,3(5,7) \\
18,9(16,6 ; 21,8)\end{array}$ & $\begin{array}{c}17,6(4,6) \\
17,2(14,6 ; 20,6)\end{array}$ & $0,001^{t}$ \\
\hline $\mathrm{VO}_{2}(\mathrm{LA}) \%$ predito & $\begin{array}{c}37,1(9,5) \\
36,1(31,4 ; 41,9)\end{array}$ & $\begin{array}{c}33,8(9,3) \\
33,7(27,8 ; 38,0)\end{array}$ & $0,046^{t}$ \\
\hline $\mathrm{VO}_{2}$ máx $\%$ predito & $\begin{array}{c}64,6(15,4) \\
62,5(53,8 ; 75,2)\end{array}$ & $\begin{array}{c}60,6(10,8) \\
60,8(52,0 ; 67,0)\end{array}$ & $0,016^{t}$ \\
\hline Pulso $\mathrm{O}_{2}$ pico, ml.bat $^{-1}$ & $\begin{array}{c}9,5(3,4) \\
9,0(7,0 ; 12,0)\end{array}$ & $\begin{array}{c}10,1(3,1) \\
9,0(8,0 ; 12,0)\end{array}$ & $0,090^{w}$ \\
\hline$\Delta$ Pulso $\mathrm{O}_{2}$ pico, $\%$ & $\begin{array}{r}9,6(2 \\
9,5(-9,6\end{array}$ & $\begin{array}{l}5,4) \\
; 25,0)\end{array}$ & \\
\hline $\begin{array}{l}\text { Pulso } \mathrm{O}_{2} \text { máx predito, } \\
\text { ml.bat }^{-1}\end{array}$ & $\begin{array}{c}12,4(3,3) \\
13,0(10,0 ; 15,0)\end{array}$ & $\begin{array}{c}12,4(3,3) \\
12,5(9,8 ; 15,0)\end{array}$ & \\
\hline $\mathrm{Vd} / \mathrm{Vt}$ repouso & $\begin{array}{c}0,29(0,14) \\
0,33(0,16 ; 0,40)\end{array}$ & $\begin{array}{c}0,23(0,12) \\
0,20(0,15 ; 0,30)\end{array}$ & $0,008^{t}$ \\
\hline
\end{tabular}




\begin{tabular}{|c|c|c|c|}
\hline $\begin{array}{l}\text { TCPE } \\
\text { BICICLETA }\end{array}$ & $\begin{array}{c}\text { PRÉ-OP } \\
\text { Média (DP) } \\
\text { Mediana (Per25; Per75) }\end{array}$ & $\begin{array}{c}\text { PÓS-OP } \\
\text { Média (DP) } \\
\text { Mediana (Per25; Per75) }\end{array}$ & $\mathbf{p}$ \\
\hline Vd/Vt pico & $\begin{array}{c}0,16(0,07) \\
0,15(0,11 ; 0,21)\end{array}$ & $\begin{array}{c}0,13(0,05) \\
0,12(0,10 ; 0,17)\end{array}$ & $0,003^{\mathrm{W}}$ \\
\hline RV, \% & $\begin{array}{c}48,4(15,9) \\
48,8(36,5 ; 61,5)\end{array}$ & $\begin{array}{c}53,3(13,5) \\
54,2(46,2 ; 61,8)\end{array}$ & $0,082^{t}$ \\
\hline $\mathrm{T} 1 / 2 \mathrm{VO}_{2}$, seg. & $\begin{array}{c}107,5(44,8) \\
100,0(77,0 ; 130,0)\end{array}$ & $\begin{array}{c}107,2(31,5) \\
105,0(85,0 ; 130,0)\end{array}$ & $0,929^{t}$ \\
\hline$\Delta \mathrm{T}^{1} 1 / 2 \mathrm{VO}_{2}, \%$ & \multicolumn{3}{|c|}{$\begin{array}{c}9,2(42,3) \\
5,6(-17,6 ; 25,9)\end{array}$} \\
\hline $\mathrm{VE} / \mathrm{VCO}_{2}$ slope & $\begin{array}{c}34,94(9,95) \\
33,17(28,0 ; 37,3)\end{array}$ & $\begin{array}{c}32,42(5,40) \\
31,00(29,0 ; 34,6)\end{array}$ & $0,078^{t}$ \\
\hline$\Delta \mathrm{VE} / \mathrm{VCO}_{2}$ slope, $\%$ & \multicolumn{3}{|c|}{$\begin{array}{c}-2,9(17,9) \\
-2,0(-14,3 ; 7,8)\end{array}$} \\
\hline OUES & $\begin{array}{c}1530(516) \\
1506(1137 ; 1934)\end{array}$ & $\begin{array}{c}1414(415) \\
1366(1079 ; 1675)\end{array}$ & $0,001^{t}$ \\
\hline$\Delta$ OUES, $\%$ & \multicolumn{3}{|c|}{$\begin{array}{c}-4,7(17,3) \\
-5,9(-16,9 ; 4,6)\end{array}$} \\
\hline OUES \% predito & $\begin{array}{c}66,7(13,9) \\
65,3(58,3 ; 74,1) \\
\end{array}$ & $\begin{array}{c}62,3(10,4) \\
62,7(53,8 ; 69,5) \\
\end{array}$ & $0,002^{t}$ \\
\hline
\end{tabular}

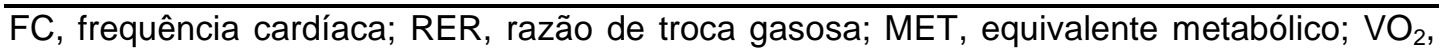
consumo de oxigênio; LA, limiar anaeróbio; $\mathrm{RV}$, reserva ventilatória; $\mathrm{Vd} / \mathrm{Vt}$, relação espaço morto ventilatório/volume corrente; $\mathrm{VE} / \mathrm{VCO}_{2}$ slope, inclinação do equivalente de ventilação do $\mathrm{CO}_{2}$; OUES, Oxygen Uptake Efficiency Slope; $\Delta$, diferença relativa; t, teste t-pareado; W, teste de Wilcoxon.

A análise da relação $\mathrm{VO}_{2} /$ carga durante o esforço nos TCPE realizados na bicicleta no momento pré-operatório foi possível em 61 pacientes e considerada normal em 59 pacientes (96,7\%) e em dois pacientes $(3,3 \%)$ foram observadas padrão de queda. No momento pósoperatório, 58 pacientes $(95,1 \%)$ apresentaram resposta normal, um paciente $(1,6 \%)$ evoluiu com platô e dois pacientes $(3,3 \%)$ com queda durante o esforço.

Antes da cirurgia, a análise da curva do pulso de $\mathrm{O}_{2}$ durante o esforço foi possível em 60 pacientes, dos quais 50 pacientes $(83,3 \%)$ apresentaram comportamento normal, quatro pacientes $(6,7 \%)$ evoluíram com platô e seis 
pacientes (10\%) com queda. Após a cirurgia, de um total de 61 pacientes em que foi possível a análise da curva, 50 pacientes (82\%) apresentaram padrão normal, cinco pacientes $(8,2 \%)$ com padrão em platô e seis pacientes $(9,8 \%)$ com padrão de queda durante o esforço.

A análise pré e pós-operatórias das curvas $\mathrm{VO}_{2} /$ carga e pulso de $\mathrm{O}_{2}$ estão indicadas na tabela 10.

Tabela 10 - Avaliação da curva $\mathrm{VO}_{2} /$ carga e pulso de $\mathrm{O}_{2}$ durante o esforço nos TCPE realizados na bicicleta antes e após a cirurgia

\begin{tabular}{lccc}
\hline & \multicolumn{2}{c}{ TCPE bicicleta } & \\
& Pré-op. & Pós-op. & $\mathbf{p}$ \\
& $\mathbf{N}(\%)$ & $\mathbf{N}(\%)$ & ANOVA NP \\
\hline Curva $\mathrm{VO}_{2}$ /carga & & & \\
Normal & $59(96,7)$ & $58(95,1)$ & \\
Platô & $0(0,0)$ & $1(1,6)$ & 0,642 \\
Queda & $2(3,3)$ & $2(3,3)$ & \\
Curva pulso de $\mathrm{O}_{2}$ & & & \\
Normal & $50(83,3)$ & $50(82,0)$ & \\
Platô & $4(6,7)$ & $5(8,2)$ & 0,846 \\
Queda & $6(10,0)$ & $6(9,8)$ & \\
\hline
\end{tabular}

TCPE, teste cardiopulmonar de exercício; $\mathrm{VO}_{2}$, consumo de oxigênio.

\subsubsection{Efeito da cirurgia nas variáveis do teste cardiopulmonar de exercício realizado na esteira}

Os TCPE na esteira foram realizados em média $31,6 \pm 29,4$ dias (mediana $=22,0$ dias) antes e 219,1 \pm 38,3 dias (mediana = 201,0 dias) após a cirurgia. 
Após a cirurgia houve significativa redução da FC pico de $152,6 \pm 26,1 \mathrm{bpm}$ para $134,6 \pm 24,7 \mathrm{bpm}(\mathrm{p}<0,001)$. $\mathrm{O} \mathrm{VO}_{2}$ pico diminuiu de $1618 \pm 579 \mathrm{~mL} \cdot \mathrm{min}^{-1}$ para $1536 \pm 447 \mathrm{~mL} \cdot \mathrm{min}^{-1}(p=0,06)$. O pulso de $\mathrm{O}_{2}$ apresentou aumento significativo após a cirurgia de 10,7 $\pm 4,0 \mathrm{~mL}$.bat ${ }^{-1}$ para $11,5 \pm 3,2 \mathrm{~mL}$.bat ${ }^{-1}(\mathrm{p}=0,023)$ e a variável $\mathrm{VE} / \mathrm{VCO}_{2}$ slope reduziu de $36,1 \pm 9,9$ para $34,5 \pm 5,6(p=0,718)$. Houve redução significativa do OUES após a cirurgia de 1760,6 $\pm 584,7$ para 1690,5 $\pm 508,4(p=0,05)$.

O T1/2 apresentou significativo aumento após a cirurgia, de $123,2 \pm 41,5$ para $133,9 \pm 36,5$ seg. $(p=0,01)$.

Os resultados pré e pós-operatórios das variáveis do TCPE e da variação percentual das diferenças do $\mathrm{VO}_{2}$ pico, pulso $\mathrm{O}_{2}$ pico, $\mathrm{T}_{1 / 2}$, $\mathrm{VE} / \mathrm{VCO}_{2}$ slope e OUES realizados na esteira ergométrica foram comparados, conforme a tabela 11.

Tabela 11 - Efeito da cirurgia nas variáveis do TCPE realizados na esteira

\begin{tabular}{lccc}
\hline TCPE & PRÉ-OP & PÓS-OP & \\
ESTEIRA & Média (DP) & Média (DP) & p \\
& Mediana (per25;per75) & Mediana (per25;per75) & \\
\hline Tempo de exercício, & $8,7(2,3)$ & $8,6(2,2)$ & $0,668^{\dagger}$ \\
min. & $9,3(7,3 ; 10,4)$ & $8,9(7,4 ; 10,3)$ & \\
FC repouso, bpm & $81,0(18,0)$ & $84,0(16,6)$ & $0,321^{\dagger}$ \\
& $77,5(69,5 ; 90,3)$ & $82,5(72,0 ; 94,5)$ & \\
FC pico, bpm & $152,6(26,1)$ & $134,6(24,7)$ & $<0,00 \mathbf{1}^{\dagger}$ \\
& $157,50(138,0 ; 171,0)$ & $134,0(120,8 ; 153,0)$ & \\
FC max predita, bpm & $170,6(16,9)$ & $169,1(17,3)$ & \\
& $168,5(158,3 ; 185,3)$ & $167,5(155,0 ; 183,3)$ & \\
\hline
\end{tabular}


continuação

\begin{tabular}{|c|c|c|c|}
\hline \multirow{3}{*}{$\begin{array}{l}\text { TCPE } \\
\text { ESTEIRA }\end{array}$} & PRÉ-OP & PÓS-OP & \multirow{3}{*}{$\mathbf{p}$} \\
\hline & Média (DP) & Média (DP) & \\
\hline & \multicolumn{2}{|c|}{ Mediana (per25;per75) Mediana (per25;per75) } & \\
\hline \multirow[t]{2}{*}{ RER pico } & $1,12(0,14)$ & $1,15(0,15)$ & $0,155^{t}$ \\
\hline & $1,14(1,02 ; 1,23)$ & $1,16(1,04 ; 1,28)$ & \\
\hline \multirow[t]{2}{*}{ MET pico } & $6,6(2,0)$ & $6,1(1,4)$ & $0,012^{t}$ \\
\hline & $6,40(5,6 ; 7,6)$ & $6,0(5,2 ; 6,9)$ & \\
\hline \multirow[t]{2}{*}{ MET predito } & $9,1(2,3)$ & $9,0(2,2)$ & \\
\hline & $8,8(7,5 ; 10,4)$ & $8,8(7,5 ; 10,2)$ & \\
\hline \multirow[t]{2}{*}{$\mathrm{VO}_{2}(\mathrm{LA}), \mathrm{mL} \cdot \mathrm{min}^{-1}$} & $1058(376)$ & $1021(319)$ & $0,243^{t}$ \\
\hline & $967(801 ; 1290)$ & $978(784 ; 1181)$ & \\
\hline \multirow[t]{2}{*}{$\mathrm{VO}_{2}$ pico, mL.min ${ }^{-1}$} & $1618(579)$ & $1536(447)$ & $0,060^{t}$ \\
\hline & $1538(1224 ; 1995)$ & $1384(1226 ; 1806)$ & \\
\hline \multirow[t]{2}{*}{$\Delta \mathrm{VO}_{2}$ pico, $\%$} & \multicolumn{2}{|c|}{$0,4(27,8)$} & \\
\hline & \multicolumn{2}{|c|}{$-4,3(-16,6 ; 6,2)$} & \\
\hline \multirow[t]{2}{*}{$\mathrm{VO}_{2}(\mathrm{LA}), \mathrm{mL} \cdot \mathrm{kg}^{-1} \cdot \mathrm{min}^{-1}$} & $15,1(4,4)$ & $14,3(3,4)$ & $0,073^{t}$ \\
\hline & $15,1(12,7 ; 17,5)$ & $13,7(11,5 ; 16,6)$ & \\
\hline \multirow[t]{2}{*}{$\mathrm{VO}_{2}$ pico, $\mathrm{mL} \cdot \mathrm{kg}^{-1} \cdot \mathrm{min}^{-1}$} & $23,1(6,8)$ & $21,5(4,9)$ & $0,011^{t}$ \\
\hline & $22,5(19,6 ; 26,7)$ & $20,9(18,2 ; 24,1)$ & \\
\hline \multirow[t]{2}{*}{$\mathrm{VO}_{2}(\mathrm{LA}) \%$ predito } & $48,7(13,1)$ & $46,4(11,0)$ & $0,126^{t}$ \\
\hline & $47,4(40,8 ; 57,2)$ & $45,4(39,4 ; 51,9)$ & \\
\hline \multirow[t]{2}{*}{$\mathrm{VO}_{2} \max \%$ predito } & $73,5(17,4)$ & $69,5(13,4)$ & $0,045^{t}$ \\
\hline & $75,0(58,1 ; 86,9)$ & $67,6(60,6 ; 76,7)$ & \\
\hline \multirow[t]{2}{*}{ Pulso $\mathrm{O}_{2}$ pico, mL.bat ${ }^{-1}$} & $10,7(4,0)$ & $11,5(3,2)$ & $0,023^{t}$ \\
\hline & $10,0(8,0 ; 13,0)$ & $11,0(9,0 ; 14,0)$ & \\
\hline \multirow[t]{2}{*}{$\Delta$ Pulso $\mathrm{O}_{2}$ pico, $\%$} & \multicolumn{2}{|c|}{$13,2(26,9)$} & \\
\hline & \multicolumn{2}{|c|}{$11,4(-7,4 ; 25,0)$} & \\
\hline Pulso $\mathrm{O}_{2}$ max predito, & $13,0(3,5)$ & $13,2(3,4)$ & \\
\hline ml.bat $^{-1}$ & $13,5(10,5 ; 16,0)$ & $13,5(11,0 ; 16,0)$ & \\
\hline \multirow[t]{2}{*}{$\mathrm{Vd} / \mathrm{Vt}$ repouso } & $0,39(0,06)$ & $0,36(0,11)$ & $0,052^{t}$ \\
\hline & $0,39(0,35 ; 0,42)$ & $0,39(0,32 ; 0,43)$ & \\
\hline
\end{tabular}


continuação

\begin{tabular}{|c|c|c|c|}
\hline TCPE & PRÉ-OP & PÓS-OP & \multirow{3}{*}{$\mathbf{p}$} \\
\hline \multirow[t]{2}{*}{ ESTEIRA } & Média (DP) & Média (DP) & \\
\hline & Mediana (per25;per75) & Mediana (per25;per75) & \\
\hline \multirow[t]{2}{*}{ Vd/Vt pico } & $0,19(0,05)$ & $0,18(0,06)$ & \multirow[t]{2}{*}{$0,023^{t}$} \\
\hline & $0,19(0,15 ; 0,23)$ & $0,18(0,14 ; 0,22)$ & \\
\hline \multirow[t]{2}{*}{$\mathbf{R V}, \%$} & $37,7(14,5)$ & $40,7(15,5)$ & \multirow[t]{2}{*}{$0,220^{t}$} \\
\hline & $37,6(26,8 ; 47,2)$ & $42,3(31,4 ; 53,2)$ & \\
\hline \multirow[t]{2}{*}{$\mathrm{T} 1 / 2 \mathrm{VO}_{2}$, seg. } & $123,2(41,5)$ & $133,9(36,5)$ & \multirow[t]{2}{*}{$0,010^{t}$} \\
\hline & $120,0(90,0 ; 145,0)$ & $135,0(105,0 ; 160,0)$ & \\
\hline \multirow[t]{2}{*}{$\Delta \mathrm{T} 1 / 2 \mathrm{VO}_{2}, \%$} & \multicolumn{2}{|c|}{$13,9(31,9)$} & \\
\hline & \multicolumn{2}{|c|}{$9,2(-7,0 ; 31,6)$} & \\
\hline \multirow[t]{2}{*}{$\mathrm{VE} / \mathrm{VCO}_{2}$ slope } & $36,1(9,9)$ & $34,5(5,6)$ & \multirow[t]{2}{*}{$0,718^{w}$} \\
\hline & $34,3(29,3 ; 39,7)$ & $33,9(30,4 ; 37,8)$ & \\
\hline \multirow[t]{2}{*}{$\Delta \mathrm{VE} / \mathrm{VCO}_{2}$ slope, $\%$} & \multicolumn{2}{|c|}{$0,6(24,2)$} & \\
\hline & \multicolumn{2}{|c|}{$0,1(-11,6 ; 17,4)$} & \\
\hline \multirow[t]{2}{*}{ OUES } & $1761(585)$ & $1691(508)$ & \multirow[t]{2}{*}{$0,050^{t}$} \\
\hline & 1624 (1334 ; 2227) & $1595(1291 ;$ 2015) & \\
\hline \multirow[t]{2}{*}{$\Delta$ OUES, $\%$} & \multicolumn{2}{|c|}{$-1,7(25,6)$} & \\
\hline & \multicolumn{2}{|c|}{$-6,4(-17,4 ; 9,5)$} & \\
\hline \multirow[t]{2}{*}{ OUES \% predito } & $78,2(16,1)$ & $74,4(14,0)$ & \multirow[t]{2}{*}{$0,043^{t}$} \\
\hline & $77,8(69,0 ; 88,2)$ & $74,0(65,1 ; 81,5)$ & \\
\hline
\end{tabular}

FC, frequência cardíaca; RER, razão de troca gasosa; $M E T$, equivalente metabólico; $\mathrm{VO}_{2}$, consumo de oxigênio; LA, limiar anaeróbio; $R V$, reserva ventilatória; $V d / V t$, relação espaço morto ventilatório/volume corrente; $\mathrm{VE} / \mathrm{VCO}_{2}$ slope, inclinação do equivalente de ventilação do $\mathrm{CO}_{2}$; OUES, Oxygen Uptake Efficiency Slope; $\Delta$, diferença relativa; t, teste t-pareado; $\mathrm{W}$, teste de Wilcoxon.

A análise da relação $\mathrm{VO}_{2} /$ carga durante 0 esforço nos TCPE realizados na esteira antes da cirurgia foi possível em 41 pacientes e, considerada normal em 36 pacientes (87,8\%), evolução com platô em quatro pacientes $(9,8 \%)$ e queda em um paciente $(2,4 \%)$. No momento pósoperatório, 50 pacientes $(84,7 \%)$ apresentaram resposta normal, sete 
pacientes $(11,9 \%)$ evoluíram com platô e dois pacientes $(3,4 \%)$ com queda durante o esforço.

A curva do pulso de $\mathrm{O}_{2}$ durante o esforço no momento pré-operatório foi realizada em 69 pacientes, dos quais 37 pacientes $(53,6 \%)$ apresentaram comportamento normal, 16 pacientes $(23,2 \%)$ evoluíram com platô e 16 pacientes $(23,2 \%)$ com queda. Após a cirurgia, a curva de $\mathrm{O} 2$ também foi avaliada em 69 pacientes, sendo que $33(47,8 \%)$ apresentaram padrão normal, 22 pacientes (31,9\%) com padrão em platô e 14 pacientes (20,3\%) evoluíram com queda durante o esforço.

A tabela 12 apresenta os resultados da análise pré e pós-operatória das curvas $\mathrm{VO}_{2} /$ carga e pulso de $\mathrm{O}_{2}$ nos TCPE realizados na esteira.

Tabela 12 - Avaliação da curva $\mathrm{VO}_{2} /$ carga e pulso de $\mathrm{O}_{2}$ durante o esforço nos TCPE realizados na esteira antes e após a cirurgia

\begin{tabular}{|c|c|c|c|}
\hline & \multicolumn{2}{|c|}{ TCPE esteira } & \multirow[b]{2}{*}{$\begin{array}{c}\text { P } \\
\text { ANOVA NP }\end{array}$} \\
\hline & $\begin{array}{l}\text { Pré-op. } \\
\text { N (\%) }\end{array}$ & $\begin{array}{c}\text { Pós-op. } \\
\text { N (\%) }\end{array}$ & \\
\hline \multicolumn{4}{|c|}{ Curva $\mathrm{VO}_{2} /$ carga } \\
\hline Normal & $36(87,8)$ & $50(84,7)$ & \\
\hline Platô & $4(9,8)$ & $7(11,9)$ & 0,575 \\
\hline Queda & $1(2,4)$ & $2(3,4)$ & \\
\hline \multicolumn{4}{|c|}{ Curva pulso de $\mathrm{O}_{2}$} \\
\hline Normal & $37(53,6)$ & $33(47,8)$ & \\
\hline Platô & $16(23,2)$ & $22(31,9)$ & 0,708 \\
\hline Queda & $16(23,2)$ & $14(20,3)$ & \\
\hline
\end{tabular}

TCPE, teste cardiopulmonar de exercício; $\mathrm{VO}_{2}$, consumo de oxigênio. 


\subsubsection{Efeito da remodelamento cardíaca após a cirurgia nas variáveis do teste cardiopulmonar de exercício: medidas de correlação}

Com o objetivo de se avaliar o impacto do remodelamento cardíaco e hemodinâmica após a cirurgia de correção da IM nos parâmetros do TCPE, a variação percentual das diferenças de resultados $(\Delta)$ de algumas variáveis dos TCPE pré e pós-operatórios $\left(\mathrm{VO}_{2}\right.$ pico, pulso de $\mathrm{O}_{2}, \mathrm{~T}_{1 / 2}, \mathrm{VE} / \mathrm{VCO}_{2}$ slope e OUES), realizados na bicicleta e na esteira, foram correlacionados com a variação percentual das diferenças das medidas ecocardiográficas pré e pós-operatórias de remodelamento cardíaco, incluindo volumes ventriculares, FE e PSAP.

Nos pacientes que realizaram o TCPE na bicicleta, o pulso de $\mathrm{O}_{2}$ e o OUES apresentaram significativa correlação com a FE e PSAP, respectivamente (Figura 8). Não houve correlação do $\mathrm{VO}_{2}, \mathrm{~T}_{1 / 2}$ e $\mathrm{VE} / \mathrm{VCO}_{2}$ slope com nenhuma medida de remodelamento cardíaco. Os resultados dessas correlações estão indicados na tabela 13.
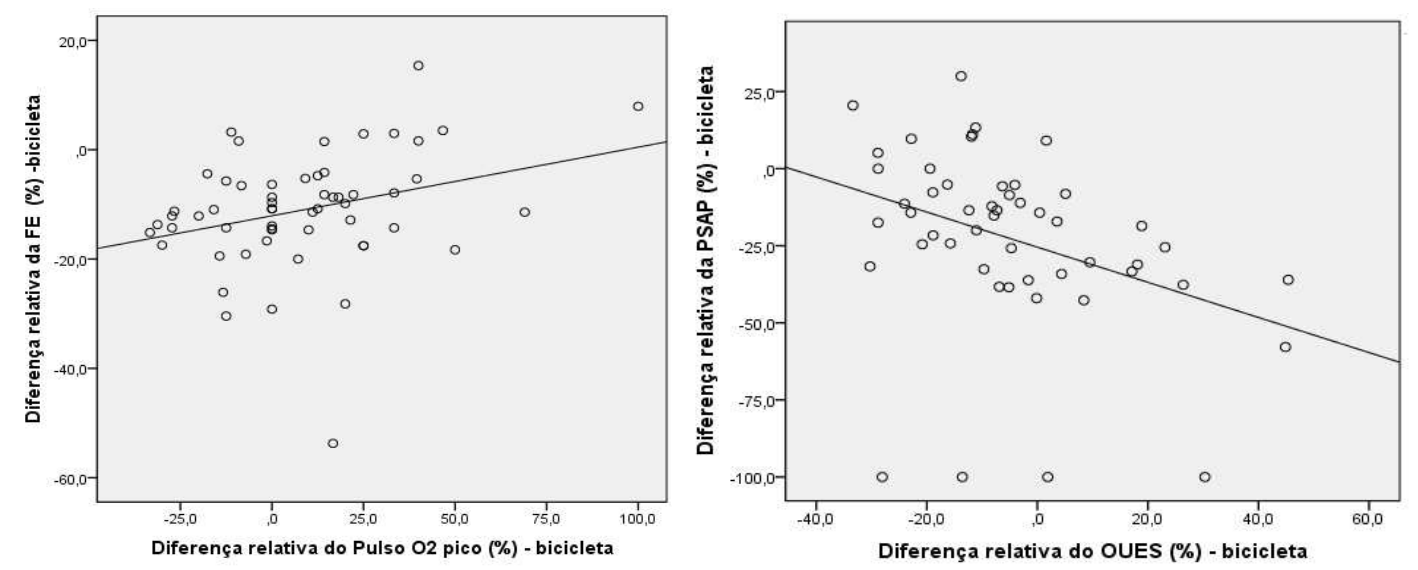

Figura 8 - Gráficos da dispersão dos valores entre diferenças relativas de medidas do ECO e variáveis do TCPE realizado na bicicleta. 
Tabela 13 - Efeito do remodelamento cardíaco nas variáveis do TCPE realizados na bicicleta: correlação entre as diferenças relativas do ECO e TCPE

TCPE bic. $x$ ECO

$\Delta$ VDFVE $\Delta$ VSFVE $\quad \Delta$ FE $\quad \Delta$ VAE $\quad \Delta$ PSAP

\begin{tabular}{lcccccc}
\hline$\Delta \mathrm{VO}_{2}$ pico, \% & $\mathrm{r}$ & $-0,039$ & $-0,041$ & 0,077 & $-0,155$ & $-0,152$ \\
& $\mathrm{p}$ & 0,764 & 0,749 & 0,551 & 0,230 & 0,264 \\
$\Delta$ Pulso $\mathrm{O}_{2}$ pico, \% & $\mathrm{r}$ & 0,024 & $-0,134$ & 0,305 & $-0,083$ & $-0,100$ \\
& $\mathrm{p}$ & 0,857 & 0,320 & $\mathbf{0 , 0 2 1}$ & 0,539 & 0,484 \\
$\mathbf{\Delta T} 1 / 2 \mathbf{V O}_{2}, \%$ & $\mathrm{r}$ & 0,032 & $-0,011$ & 0,086 & 0,171 & 0,162 \\
& $\mathrm{p}$ & 0,807 & 0,931 & 0,508 & 0,187 & 0,238
\end{tabular}

$\begin{array}{lllllll}\Delta \mathrm{VE} / \mathrm{VCO}_{2} \text { slope, } \% & r & 0,201 & 0,133 & 0,013 & 0,121 & 0,186\end{array}$

$p \quad 0,121 \quad 0,307 \quad 0,921 \quad 0,355 \quad 0,174$

$\begin{array}{lllllll}\Delta \text { OUES, } \% & \mathrm{r} & -0,004 & -0,093 & 0,182 & -0,104 & -0,359\end{array}$

$\begin{array}{llllll}p & 0,974 & 0,492 & 0,175 & 0,440 & \mathbf{0 , 0 1 0}\end{array}$

VDFVE, volume diastólico final do ventrículo esquerdo; VSFVE, volume sistólico final do ventrículo esquerdo; FE, fração de ejeção; VAE, volume do átrio esquerdo; PSAP, pressão sistólica em artéria pulmonar, $\mathrm{VO}_{2}$, consumo de oxigênio; $\mathrm{VE} / \mathrm{VCO}_{2}$ slope, inclinação do equivalente de ventilação do $\mathrm{CO}_{2}$; OUES, Oxygen Uptake Efficiency Slope; $\Delta$, diferença relativa; r, coeficiente de correlação de Pearson.

$\mathrm{Na}$ análise dos TCPE realizados na esteira, o pulso de $\mathrm{O}_{2}, \mathrm{~T}_{1 / 2}$ e $\mathrm{o}$ $\mathrm{VE} / \mathrm{VCO}_{2}$ slope não apresentaram correlação com as medidas ecocardiográficas de remodelamento cardíaco. Entretanto, o $\mathrm{VO}_{2}$ pico apresentou significativa correlação negativa com a PSAP, assim como o OUES com o VDFVE, VAE e a PSAP (Figura 9). A tabela 14 mostra os resultados dessas correlações. 

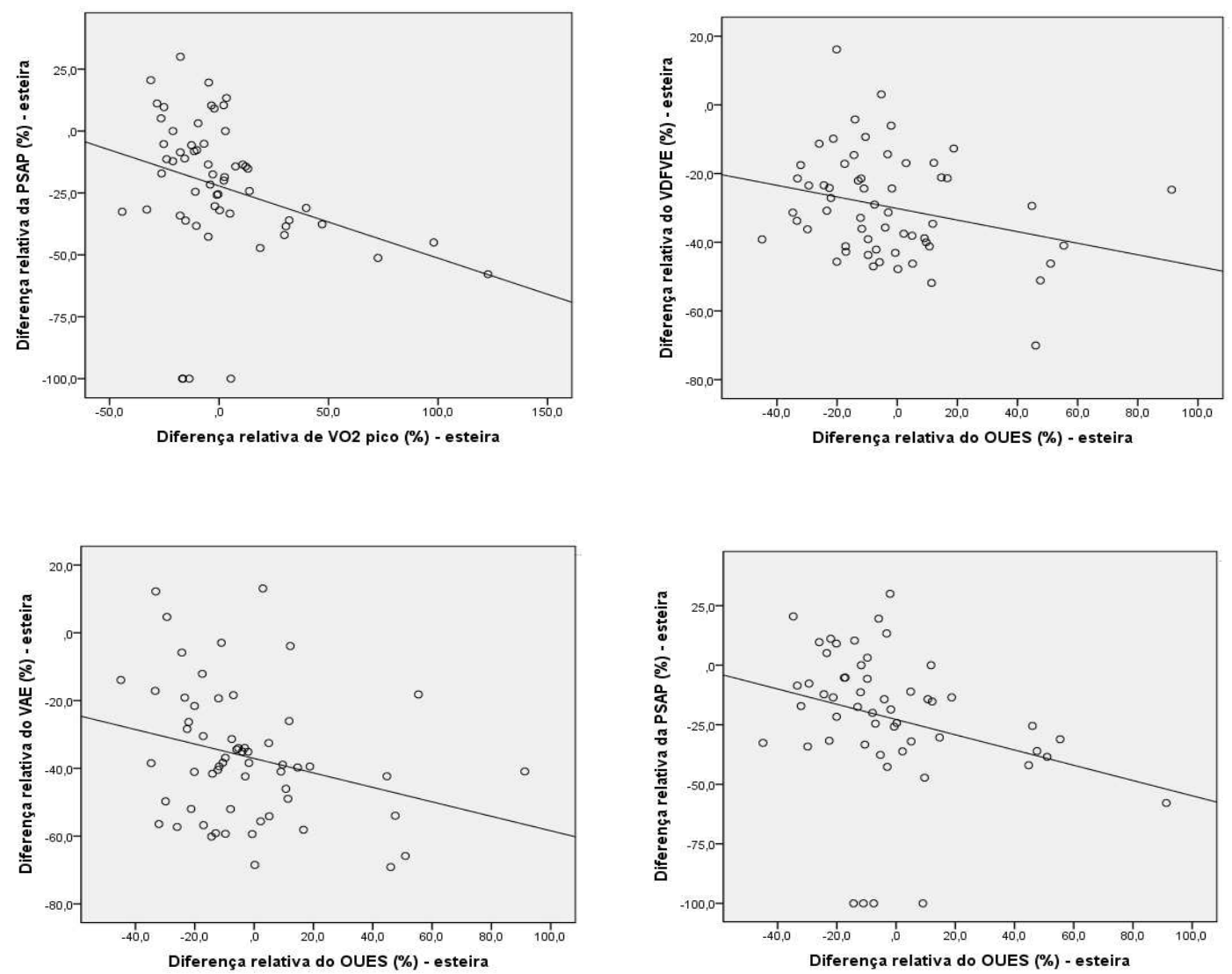

Figura 9 - Gráficos da dispersão dos valores entre diferenças relativas de medidas do ECO e variáveis do TCPE realizado na esteira. 
Tabela 14 - Efeito do remodelamento cardíaco nas variáveis do TCPE realizados na esteira: correlação entre as diferenças relativas do ECO e TCPE

\begin{tabular}{|c|c|c|c|c|c|c|}
\hline TCPE est. x ECO & & $\Delta$ VDFVE & $\Delta$ VSFVE & $\Delta \mathrm{FE}$ & $\Delta$ VAE & $\triangle \mathrm{PSAF}$ \\
\hline \multirow[t]{2}{*}{$\Delta \mathrm{VO}_{2}$ pico, $\%$} & $r$ & $-0,035$ & 0,000 & $-0,071$ & $-0,236$ & $-0,298$ \\
\hline & $p$ & 0,779 & 0,998 & 0,574 & 0,058 & 0,023 \\
\hline \multirow[t]{2}{*}{$\Delta$ Pulso $\mathrm{O}_{2}$ pico, $\%$} & $r$ & 0,093 & $-0,009$ & 0,149 & $-0,090$ & $-0,041$ \\
\hline & $\mathrm{p}$ & 0,463 & 0,945 & 0,236 & 0,475 & 0,758 \\
\hline \multirow[t]{2}{*}{$\Delta \mathrm{T} 1 / 2 \mathrm{VO}_{2}, \%$} & r & $-0,042$ & $-0,054$ & $-0,009$ & 0,087 & 0,082 \\
\hline & $p$ & 0,745 & 0,677 & 0,948 & 0,504 & 0,546 \\
\hline \multirow[t]{2}{*}{$\Delta \mathrm{VE} / \mathrm{VCO}_{2}$ slope, $\%$} & $r$ & 0,029 & 0,105 & $-0,181$ & 0,155 & 0,158 \\
\hline & $\mathrm{p}$ & 0,822 & 0,404 & 0,148 & 0,217 & 0,238 \\
\hline \multirow[t]{2}{*}{$\triangle$ OUES, $\%$} & r & $-0,277$ & $-0,209$ & 0,059 & $-0,275$ & $-0,282$ \\
\hline & $p$ & 0,034 & 0,112 & 0,657 & 0,035 & 0,041 \\
\hline
\end{tabular}

VDFVE, volume diastólico final do ventrículo esquerdo; VSFVE, volume sistólico final do ventrículo esquerdo; FE, fração de ejeção; VAE, volume do átrio esquerdo; PSAP, pressão sistólica em artéria pulmonar, $\mathrm{VO}_{2}$, consumo de oxigênio; $\mathrm{VE} / \mathrm{VCO}_{2}$ slope, inclinação do equivalente de ventilação do $\mathrm{CO}_{2}$; OUES, Oxygen Uptake Efficiency Slope; $\Delta$, diferença relativa; $r$, coeficiente de correlação de Pearson; $r$, coeficiente de correlação de Spearman.

\subsection{Grupos troca valvar mitral e reparo mitral}

Os pacientes da população em estudo foram separados em dois grupos de acordo com o tipo de cirurgia corretiva da IM realizada: troca valvar mitral (TVM) ou reparo mitral (RM). Os resultados da avaliação comparativa entre ambos os grupos estão descritos a seguir. 


\subsubsection{Características de base}

Nos 47 pacientes submetidos ao RM, houve predominância do sexo masculino (30 pacientes - 63,8\%) com média das idades de 48,5 $\pm 17,5$ anos e a etiologia mais frequente foi a degenerativa em 35 pacientes $(74,5 \%)$.

No grupo TVM não houve predominância com relação ao sexo (masculino em 12 pacientes - 48\%), a média de idades foi de $52,5 \pm 15,9$ anos e a etiologia predominante foi à degenerativa em 15 pacientes (60\%).

Não houve diferença significativa na CF entre ambos os grupos. A média da CF no grupo TVM foi de $2,1 \pm 0,8$ e no RM de $2,0 \pm 0,8$.

As principais características de base dos dois grupos foram comparadas e estão expressas em frequência (n e \%) e mediana conforme mostra a tabela 15 . 
Tabela 15 - Características de base em pacientes submetidos à troca valvar mitral e reparo mitral

\begin{tabular}{|c|c|c|c|}
\hline & $\begin{array}{c}\text { TVM } \\
(n=25)\end{array}$ & $\begin{array}{c}\mathbf{R M} \\
(\mathrm{n}=47)\end{array}$ & p \\
\hline $\begin{array}{l}\text { Sexo masculino } \\
\text { Idade (anos) }\end{array}$ & $\begin{array}{c}12(48 \%) \\
52,5 \pm 15,9 \\
53,0\end{array}$ & $\begin{array}{c}30(63,8 \%) \\
48,5 \pm 17,5 \\
52,0\end{array}$ & $\begin{array}{l}0,195^{P} \\
0,347^{t}\end{array}$ \\
\hline \multicolumn{4}{|l|}{ Etiologia } \\
\hline $\begin{array}{l}\text { PVM } \\
\text { Reumática } \\
\text { Outras }\end{array}$ & $\begin{array}{c}15(60,0 \%) \\
10(40,0 \%) \\
0(0,0 \%)\end{array}$ & $\begin{array}{c}35(74,5 \%) \\
10(21,3 \%) \\
2(4,2 \%)\end{array}$ & $0,243^{\mathrm{F}}$ \\
\hline CF NYHA & $\begin{array}{c}2,1 \pm 0,8 \\
2,0\end{array}$ & $\begin{array}{c}2,0 \pm 0,8 \\
2,0\end{array}$ & \multirow{5}{*}{$0,995^{\vdash}$} \\
\hline I & $6(24,0 \%)$ & $13(27,7 \%)$ & \\
\hline II & $11(44,0 \%)$ & $21(44,7 \%)$ & \\
\hline III & $7(28,0 \%)$ & $12(25,5 \%)$ & \\
\hline IV & $1(4,0 \%)$ & $1(2,1 \%)$ & \\
\hline \multicolumn{4}{|l|}{ ECG } \\
\hline Fibrilação atrial & $5(20,0 \%)$ & $3(6,4 \%)$ & $0,080^{P}$ \\
\hline Hipertensão & $16(64,0 \%)$ & $23(48,9 \%)$ & $0,222^{P}$ \\
\hline Diabetes & $3(12,0 \%)$ & $3(6,4 \%)$ & $0,412^{\mathrm{F}}$ \\
\hline Obesidade & $3(12,0 \%)$ & $11(23,4 \%)$ & $0,244^{P}$ \\
\hline Fumante ativo & $2(8,0 \%)$ & $4(8,5 \%)$ & $1,000^{\mathrm{F}}$ \\
\hline $\begin{array}{l}\text { Dislipidemia } \\
\text { Medicamentos em }\end{array}$ & $8(32.0 \%)$ & $7(14,9 \%)$ & $0,089^{P}$ \\
\hline Diurético & $11(44,0 \%)$ & $21(44,7 \%)$ & $0,947^{P}$ \\
\hline Betabloqueador & $13(52,0 \%)$ & $17(36,2 \%)$ & $0,191^{\mathrm{P}}$ \\
\hline IECA & $14(56,0 \%)$ & $22(46,8 \%)$ & $0,454^{P}$ \\
\hline BRA & $2(8,0 \%)$ & $3(6,4 \%)$ & $1,000^{F}$ \\
\hline Amiodarona & $2(8,0 \%)$ & $2(4,3 \%)$ & $0,606^{F}$ \\
\hline
\end{tabular}

PVM, prolapso da valva mitral; CF, classe funcional; NYHA, New York Heart Association; IECA, inibidor da enzima conversora da angiotensina; BRA, bloqueador do receptor da angiotensina 1; P: teste de Pearson; F: teste de Fisher; t: teste $t$ de student.

A análise bioquímica não mostrou diferenças significativas entre os grupos, conforme pode ser observado na tabela 16. 
Tabela 16 - Comparação dos exames laboratoriais pré-operatórios em pacientes submetidos à troca valvar mitral e reparo mitral

\begin{tabular}{|c|c|c|c|}
\hline & $\begin{array}{c}\text { TVM } \\
\text { Média (DP) } \\
\text { Mediana (per75;per75) }\end{array}$ & $\begin{array}{c}\text { RM } \\
\text { Média (DP) } \\
\text { Mediana (per25;per75) }\end{array}$ & p \\
\hline \multirow[t]{2}{*}{ Glicemia, mg.dL ${ }^{-1}$} & $85,2(12,8)$ & $87,6(14,1)$ & $0,599^{\mathrm{M}}$ \\
\hline & $85,0(74,5 ; 92,5)$ & $86,0(77,0 ; 98,0)$ & \\
\hline \multirow[t]{2}{*}{ Ureia, mg.dL ${ }^{-1}$} & $36,9(10,9)$ & $36,4(9,8)$ & $0,836^{t}$ \\
\hline & $36,0(26,0 ; 40,0)$ & $35,0(28,0 ; 42,0)$ & \\
\hline \multirow[t]{2}{*}{ Creatinina, mg.dL ${ }^{-1}$} & $0,91(0,25)$ & $0,98(0,27)$ & $0,153^{\mathrm{M}}$ \\
\hline & $0,80(0,75 ; 1,05)$ & $0,95(0,80 ; 1,12)$ & \\
\hline \multirow[t]{2}{*}{ Hemoglobina, g.dL $\mathrm{dL}^{-1}$} & $14,1(1,4)$ & $14,3(1,5)$ & $0,524^{t}$ \\
\hline & $13,8(13,4 ; 14,8)$ & $14,0(13,3 ; 15,0)$ & \\
\hline \multirow[t]{2}{*}{ Hematócrito, \% } & $42,2(3,7)$ & $42,7(4,5)$ & $0,741^{\mathrm{M}}$ \\
\hline & $42,3(39,5 ; 44,7)$ & $42,0(40,1 ; 45,7)$ & \\
\hline
\end{tabular}

t, teste t de student; M, Mann-Whitney.

O grupo reparo mitral apresentou menor volume atrial esquerdo préoperatório do que o grupo troca valvar mitral $(p=0,092)$. As demais médias dos parâmetros ecocardiográficos não apresentaram diferenças significativas em ambos os grupos. A tabela 17 apresenta a comparação ecocardiográfica pré-operatória no grupo troca valvar mitral e reparo mitral. 
Tabela 17 - Comparação pré-operatória dos parâmetros ecocardiográficos em pacientes submetidos à troca valvar mitral e reparo mitral

\begin{tabular}{lccc}
\hline ECO & TVM & RM & \\
Pré-operatório & $\begin{array}{c}\text { Média (DP) } \\
\text { Mediana (per25;per75) }\end{array}$ & $\begin{array}{c}\text { Média (DP) } \\
\text { Mediana (per25;per75) }\end{array}$ & p \\
\hline VAE, mm & $146,8(86,8)$ & $112,7(32,5)$ & $0,092^{\mathrm{M}}$ \\
& $133,5(88,0 ; 170,5)$ & $107,8(92,8 ; 128,5)$ & \\
DDFVE, mm & $61,1(5,4)$ & $61,8(4,5)$ & $0,924^{\mathrm{M}}$ \\
& $61,5(57,3 ; 64,0)$ & $61,5(58,3 ; 64,0)$ & \\
DSFVE, mm & $38,7(4,8)$ & $38,8(4,3)$ & $0,793^{\mathrm{M}}$ \\
& $38,5(36,0 ; 40,0)$ & $38,0(36,3 ; 40,0)$ & \\
VDFVE, mL & $145,0(41,8)$ & $154,2(32,4)$ & $0,505^{\mathrm{M}}$ \\
& $148,0(106,0 ; 179,0)$ & $152,5(132,0 ; 171,3)$ & \\
VSFVE, mL & $47,4(17,9)$ & $47,7(18,3)$ & $0,984^{\mathrm{M}}$ \\
& $44,0(36,0 ; 62,0)$ & $43,5(37,3 ; 54,0)$ & \\
FE, \% & $68,1(6,7)$ & $69,6(6,2)$ & $0,347^{\mathrm{M}}$ \\
& $69,0(63,0 ; 73,0)$ & $70,0(67,0 ; 74,0)$ & \\
PSAP, mm Hg & $48,4(15,5)$ & $45,7(15,1)$ & $0,577^{\mathrm{M}}$ \\
& $46,0(36,0 ; 53,0)$ & $41,5(35,8 ; 49,3)$ & \\
\hline
\end{tabular}

VDFVE, volume diastólico final do ventrículo esquerdo; VSFVE, volume sistólico final do ventrículo esquerdo; DDFVE, diâmetro diastólico final do ventrículo esquerdo; DSFVE, diâmetro sistólico final do ventrículo esquerdo; FE, fração de ejeção; VAE, volume do átrio esquerdo; PSAP, pressão sistólica em artéria pulmonar; M, Mann-Whitney.

As medidas pressóricas e de função ventricular esquerda realizada pelo cateterismo cardíaco, no momento pré-operatório, não apresentaram diferenças significativas nos grupos TVM e RM, conforme pode ser observado na tabela 18. 
Tabela 18 - Cateterismo cardíaco: comparação pré-operatória entre a troca valvar mitral e o reparo mitral

\begin{tabular}{lccc}
\hline & $\begin{array}{c}\text { Troca Valvar Mitral } \\
\text { Média (DP) }\end{array}$ & $\begin{array}{c}\text { Reparo Mitral } \\
\text { Média (DP) }\end{array}$ & Valor de p \\
& Mediana (per25;per75) & Mediana (per25;per75) & \\
\hline PCP, mmHg & $22,4(17,2)$ & $18,3(8,5)$ & $0,972^{\mathrm{M}}$ \\
& $17,0(12,0 ; 25,0)$ & $18,0(10,0 ; 25,0)$ & \\
PDVE, mmHg & $17,2(16,8)$ & $15,2(7,1)$ & $0,731^{\mathrm{M}}$ \\
& $12,0(10,0 ; 20,5)$ & $15,0(10,0 ; 20,0)$ & \\
ADm, mmHg & $7,9(4,2)$ & $7,1(4,8)$ & $0,429^{\mathrm{M}}$ \\
& $8,5(4,8 ; 10,0)$ & $6,0(3,0 ; 11,0)$ & \\
PSAP, mmHg & $40,6(14,8)$ & $38,2(13,3)$ & $0,822^{\mathrm{M}}$ \\
& $35,5(30,8 ; 49,0)$ & $38,0(26,0 ; 47,0)$ & \\
VDFVE, mL & $182,7(65,1)$ & $189,3(70,0)$ & $0,886^{\mathrm{M}}$ \\
& $174,4(138,1 ; 226,5)$ & $166,5(146,1 ; 211,0)$ & \\
VSFVE, mL & $57,4(33,6)$ & $63,5(42,7)$ & $0,567^{\mathrm{M}}$ \\
& $47,5(33,4 ; 69,9)$ & $56,4(34,1 ; 80,3)$ & \\
FE, \% & $68,5(9,2)$ & $68,2(13,2)$ & $0,579^{\mathrm{M}}$ \\
& $69,2(61,3 ; 75,0)$ & $71,6(61,9 ; 77,7)$ & \\
\hline
\end{tabular}

PCP, pressão capilar pulmonar; PDVE, pressão diastólica final do VE; ADm, pressão média em átrio direito; PSAP, pressão sistólica em artéria pulmonar; VDFVE, volume diastólico final do VE; VSFVE, volume sistólico final do VE; FE, fração de ejeção; M, Mann-Whitney.

\subsubsection{Efeito da cirurgia nos parâmetros ecocardiográficos}

As medidas ecocardiográficas de remodelamento cardíaco nos grupos TVM e RM foram analisadas e comparadas.

Houve significativa redução dos volumes atrial e ventricular esquerdo, da FE e da PSAP após a cirurgia em ambos os grupos sendo que o grupo 
RM apresentou menor VAE antes e após a cirurgia. As demais medidas ecocardiográficas não apresentaram diferenças nos grupos TVM e RM, nos momentos pré e pós-operatório.

A tabela 19 mostra a análise e comparação das medidas ecocardiográficas de remodelamento cardíaco nos grupos troca valvar mitral e reparo mitral.

Tabela 19 - Comparação ecocardiográfica das medidas de remodelação nos grupos e entre os grupos troca valvar mitral e reparo mitral

\begin{tabular}{|c|c|c|c|c|c|c|c|c|}
\hline & & TVM & & & $\mathbf{R M}$ & & Pré & Pós \\
\hline & Pré & Pós & p & Pré & Pós & $\mathbf{p}$ & TVM & $T V M$ \\
\hline & $\begin{array}{c}\text { Média (DP) } \\
\text { Mediana }\end{array}$ & $\begin{array}{c}\text { Média (DP) } \\
\text { Mediana }\end{array}$ & & $\begin{array}{c}\text { Média (DP) } \\
\text { Mediana }\end{array}$ & $\begin{array}{c}\text { Média (DP) } \\
\text { Mediana }\end{array}$ & & $\begin{array}{c}X \\
R M\end{array}$ & $\begin{array}{c}X \\
R M\end{array}$ \\
\hline VAE, $\mathrm{mm}^{3}$ & $\begin{array}{c}146,8(86,8) \\
133,5\end{array}$ & $\begin{array}{c}92,0(37,5) \\
79,0\end{array}$ & $<0,001^{w}$ & $\begin{array}{c}112,7(32,5) \\
107,8\end{array}$ & $\begin{array}{c}68,1(29,1) \\
63,5\end{array}$ & $<0,001^{w}$ & $0,092^{m}$ & $0,004^{m I}$ \\
\hline$\Delta$ VAE, \% & & $\begin{array}{c}-32,4(18,6) \\
-34,4\end{array}$ & & & $\begin{array}{c}37,9(20,2) \\
-39,9\end{array}$ & & & \\
\hline DDFVE, mm & $\begin{array}{c}61,1(5,4) \\
61,5\end{array}$ & $\begin{array}{c}50,8(5,9) \\
51,0\end{array}$ & $<0,001^{\mathrm{tp}}$ & $\begin{array}{c}61,8(4,5) \\
61,5\end{array}$ & $\begin{array}{c}52,9(6,0) \\
53,0\end{array}$ & $<0,001^{\mathrm{tp}}$ & $0,576^{t}$ & $0,173^{t}$ \\
\hline$\Delta$ DDFVE, \% & & $\begin{array}{c}-16,7(8,4) \\
-16,9\end{array}$ & & & $\begin{array}{c}-14,2(7,8) \\
-14,1\end{array}$ & & & \\
\hline DSFVE, mm & $\begin{array}{c}38,7(4,8) \\
38,5\end{array}$ & $\begin{array}{c}34,0(5,7) \\
33,0\end{array}$ & $<0,001^{w}$ & $\begin{array}{c}38,8(4,3) \\
38,0\end{array}$ & $\begin{array}{c}35,8(6,6) \\
35,0\end{array}$ & $<0,001^{w}$ & $0,816^{\mathrm{M}}$ & $0,37^{M}$ \\
\hline$\Delta$ DSFVE, $\%$ & & $\begin{array}{c}-12,1(10,7) \\
-11,4\end{array}$ & & & $\begin{array}{c}7,6(12,5) \\
-9,7\end{array}$ & & & \\
\hline VDFVE, mL & $\begin{array}{c}145,0(41,8) \\
148,0\end{array}$ & $\begin{array}{c}101,7(35,1) \\
99,0\end{array}$ & ) $<0,001^{w}$ & $\begin{array}{c}154,2(32,4) \\
152,5\end{array}$ & $\begin{array}{c}113,7(46,2) \\
110,0\end{array}$ & $<0,001^{w}$ & $0,505^{\mathrm{M}}$ & $0,29^{\mathrm{M}}$ \\
\hline$\Delta$ VDFVE, $\%$ & & $\begin{array}{c}-29,0(17,3) \\
-29,7\end{array}$ & & & $\begin{array}{c}26,5(21,6) \\
-31,1\end{array}$ & & & \\
\hline VSFVE, mL & $\begin{array}{c}47,4(17,9) \\
44,0\end{array}$ & $\begin{array}{c}40,8(19,6) \\
33,0\end{array}$ & $0,036^{w}$ & $\begin{array}{c}47,7(18,3) \\
43,5\end{array}$ & $\begin{array}{c}45,5(30,4) \\
38,0\end{array}$ & $0,017^{w}$ & $0,984^{\mathrm{M}}$ & $0,344^{\mathrm{M}}$ \\
\hline$\Delta$ VSFVE, $\%$ & & $\begin{array}{c}-11,3(25,8) \\
-11,1\end{array}$ & & & $\begin{array}{c}4,8(35,0) \\
-8,1\end{array}$ & & & \\
\hline
\end{tabular}


Continuação

\begin{tabular}{|c|c|c|c|c|c|c|c|c|}
\hline & $\begin{array}{c}\text { Pré } \\
\text { Média (DP } \\
\text { Mediana }\end{array}$ & $\begin{array}{c}\text { Pós } \\
\text { Média (DP } \\
\text { Mediana }\end{array}$ & $p$ & $\begin{array}{c}\text { Pré } \\
\text { Média (DP } \\
\text { Mediana }\end{array}$ & $\begin{array}{c}\text { Pós } \\
\text { Média (DP } \\
\text { Mediana }\end{array}$ & $p$ & $\begin{array}{c}T V M \\
x \\
R M\end{array}$ & $\begin{array}{c}\text { TVM } \\
x \\
R M\end{array}$ \\
\hline FE, \% & $\begin{array}{c}68,1(6,7) \\
69,0\end{array}$ & $\begin{array}{c}61,5(7,4) \\
64,5\end{array}$ & $<0,001^{\mathrm{tp}}$ & $\begin{array}{c}69,6(6,2) \\
70,0\end{array}$ & $\begin{array}{c}61,2(8,7) \\
62,0\end{array}$ & $<0,001^{\mathrm{tp}}$ & $0,369^{t}$ & $0,894^{t}$ \\
\hline$\Delta \mathrm{FE}, \%$ & & $\begin{array}{c}-9,5(11,3) \\
-8,2\end{array}$ & & & $\begin{array}{l}1,7(12,0) \\
-11,8\end{array}$ & & & \\
\hline $\begin{array}{l}\text { PSAP, } \\
\text { mm Hg }\end{array}$ & $\begin{array}{c}48,4(15,5) \\
46,0\end{array}$ & $\begin{array}{c}36,1(14,0) \\
36,0\end{array}$ & $<0,001^{w}$ & $\begin{array}{c}45,7(15,1) \\
41,5\end{array}$ & $\begin{array}{c}35,0(11,7) \\
34\end{array}$ & $<0,001^{w}$ & $0,577^{\mathrm{M}}$ & $0,347^{\mathrm{M}}$ \\
\hline$\Delta$ PSAP, $\%$ & & $\begin{array}{c}-25,4(29,7) \\
-21,6\end{array}$ & & & $\begin{array}{l}0,8(27,9) \\
-18,6\end{array}$ & & & \\
\hline
\end{tabular}

\subsubsection{Efeito da cirurgia nas variáveis do teste cardiopulmonar de exercício}

As principais variáveis do TCPE realizados na esteira e no cicloergômetro antes e após a troca valvar mitral e o reparo mitral foram analisadas e comparadas, conforme pode ser observado nas tabelas 20 e 21.

No TCPE realizado na bicicleta, em ambos os grupos, houve significativa redução pós-operatória da FC pico, não havendo diferenças na análise entre os grupos. $\mathrm{OVO}_{2}$ pico e OUES apresentaram leve redução após a cirurgia. A relação $\mathrm{VE} / \mathrm{VCO}_{2}$ slope apresentou uma redução mais expressiva após a cirurgia no grupo TVM do que no grupo RM, com uma diferença relativa pré e pós-operatória de $-9,9 \pm 13,6 \%$. O pulso de $\mathrm{O}_{2}$ 
aumentou no momento pós-operatório em ambos os grupos, significativamente apenas no grupo RM.

O grupo RM apresentou maiores valores do $\mathrm{VO}_{2}$ pico e do OUES em relação ao grupo TVM, antes e após a cirurgia.

Tabela 20 - Efeito da cirurgia nas variáveis do TCPE realizados na bicicleta em pacientes submetidos à troca valvar mitral e reparo mitral

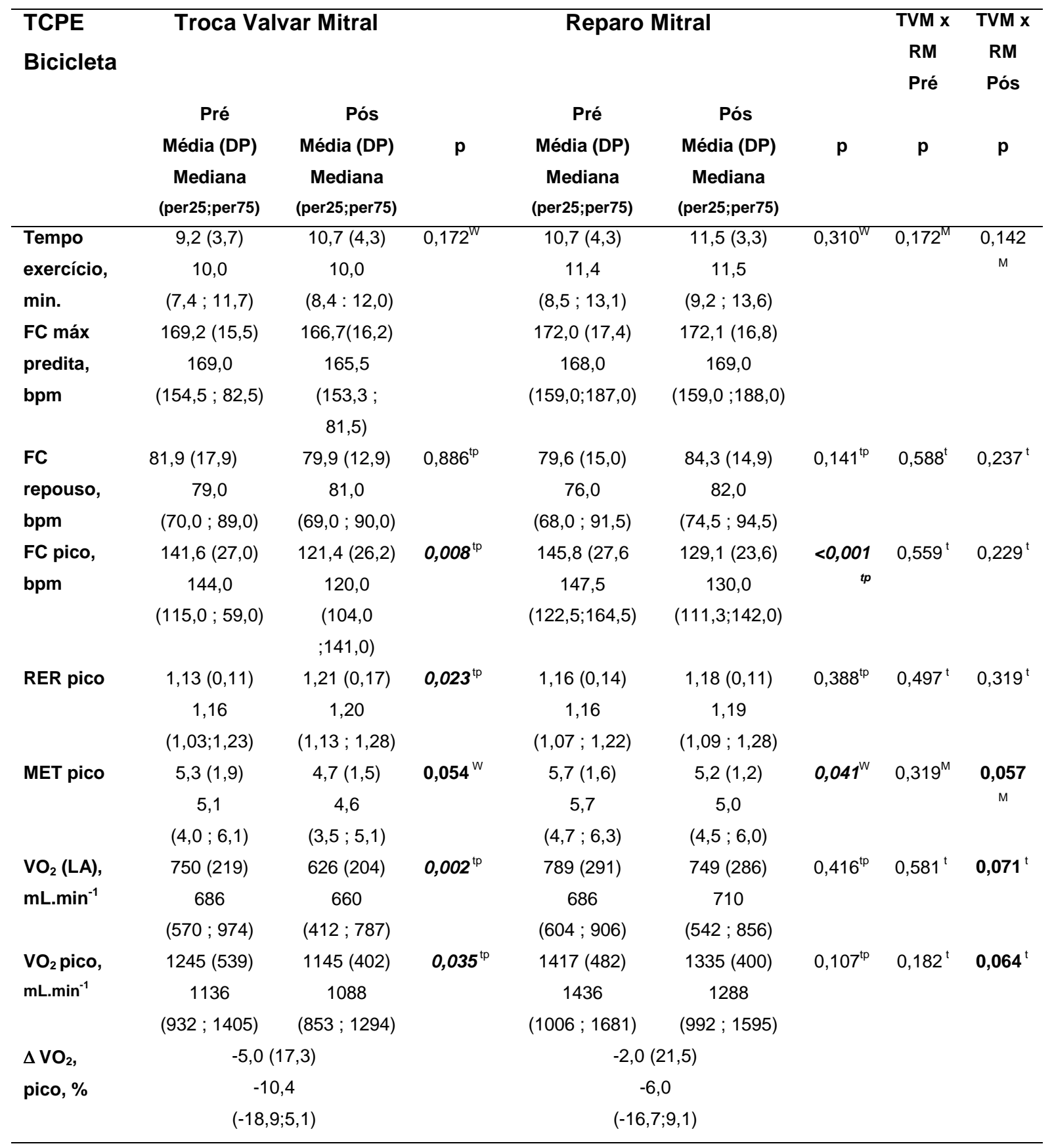




\section{continuação}

\begin{tabular}{|c|c|c|c|c|c|c|c|c|}
\hline \multirow{2}{*}{$\begin{array}{l}\text { TCPE } \\
\text { Bicicleta }\end{array}$} & \multicolumn{2}{|c|}{ Troca Valvar Mitral } & \multicolumn{4}{|c|}{ Reparo Mitral } & $\begin{array}{l}\text { TVM x } \\
\text { RM } \\
\text { Pré }\end{array}$ & $\begin{array}{l}\text { TVM x } \\
\text { RM } \\
\text { Pós }\end{array}$ \\
\hline & $\begin{array}{c}\text { Pré } \\
\text { Média (DP) } \\
\text { Mediana } \\
\text { (per25;per75) }\end{array}$ & $\begin{array}{c}\text { Pós } \\
\text { Média (DP) } \\
\text { Mediana } \\
\text { (per25;per75) }\end{array}$ & p & $\begin{array}{c}\text { Pré } \\
\text { Média (DP) } \\
\text { Mediana } \\
\text { (per25;per75) }\end{array}$ & $\begin{array}{c}\text { Pós } \\
\text { Média (DP) } \\
\text { Mediana } \\
\text { (per25;per75) }\end{array}$ & $\mathbf{p}$ & $\mathbf{p}$ & p \\
\hline $\begin{array}{l}\mathrm{VO}_{2}(\mathrm{LA}), \\
\mathrm{mL} \cdot \mathrm{kg}^{-1} \cdot \mathrm{min}^{-1}\end{array}$ & $\begin{array}{c}11,2(2,7) \\
12,3 \\
(9,2 ; 13,4)\end{array}$ & $\begin{array}{c}9,0(2,8) \\
9,0 \\
(6,3 ; 11,1)\end{array}$ & $0,001^{\mathrm{tp}}$ & $\begin{array}{c}11,1(3,0) \\
10,9 \\
(9,1 ; 12,3)\end{array}$ & $\begin{array}{c}10,2(3,1) \\
9,6 \\
(7,9 ; 11,7)\end{array}$ & $0,201^{\mathrm{tp}}$ & $0,811^{t}$ & $0,115^{t}$ \\
\hline $\begin{array}{l}\mathrm{VO}_{2} \text { pico, } \\
\mathrm{mL} \cdot \mathrm{kg}^{-1} \cdot \mathrm{min}^{-1}\end{array}$ & $\begin{array}{c}18,5(6,1) \\
18,0 \\
(16,0 ; 20,4)\end{array}$ & $\begin{array}{c}16,4(5,2) \\
16,1 \\
(12,4 ; 18,0)\end{array}$ & $0,001^{\text {tp }}$ & $\begin{array}{c}19,8(5,4 \\
19,8 \\
(16,6 ; 21,9)\end{array}$ & $\begin{array}{c}18,3(4,1) \\
17,5 \\
(15,7 ; 21,0)\end{array}$ & $0,030^{\mathrm{tp}}$ & $0,377^{\mathrm{t}}$ & $0,110^{t}$ \\
\hline $\begin{array}{l}\mathrm{VO}_{2}(\mathrm{LA}) \% \\
\text { predito }\end{array}$ & $\begin{array}{c}37,6(9,4) \\
36,4 \\
(31,9 ; 44,3)\end{array}$ & $\begin{array}{c}31,7(7,4) \\
31,8 \\
(26,9 ; 35,1)\end{array}$ & $0,003^{t p}$ & $\begin{array}{c}36,8(9,6) \\
36,1 \\
(30,1 ; 41,1)\end{array}$ & $\begin{array}{c}34,8(10,0) \\
34,5 \\
(28,8 ; 39,2)\end{array}$ & $0,488^{t p}$ & $0,734^{t}$ & $0,194^{t}$ \\
\hline $\begin{array}{l}\text { Pulso } \mathrm{O}_{2} \\
\text { pico, } \\
\text { mL.bat }^{-1}\end{array}$ & $\begin{array}{c}9,0(4,1) \\
8,0 \\
(6,5 ; 10,0)\end{array}$ & $\begin{array}{c}9,5(2,9) \\
8,0 \\
(7,0 ; 12,0)\end{array}$ & $0,919^{w}$ & $\begin{array}{c}9,8(3,0) \\
9,5 \\
(7,8 ; 12,0)\end{array}$ & $\begin{array}{c}10,5(3,2) \\
10,0 \\
(8,0 ; 12,0)\end{array}$ & $0,033^{w}$ & $0,156^{\mathrm{M}}$ & $\begin{array}{l}0,209 \\
M\end{array}$ \\
\hline $\begin{array}{l}\Delta \text { Pulso } \mathrm{O}_{2} \\
\text { pico, \% }\end{array}$ & $\begin{array}{r}9,1 \\
1 \\
(-14,6\end{array}$ & $\begin{array}{l}0,1) \\
5 \\
26,1)\end{array}$ & & $\begin{array}{r}9,9 \\
(-4,3\end{array}$ & $\begin{array}{l}3,0) \\
25,0)\end{array}$ & & & \\
\hline $\begin{array}{l}\text { Vd/Vt } \\
\text { repouso }\end{array}$ & $\begin{array}{c}0,31(0,13) \\
0,33 \\
(0,21 ; 0,40)\end{array}$ & $\begin{array}{c}0,21(0,11) \\
0,20 \\
(0,16 ; 0,23)\end{array}$ & $0,031^{\text {tp }}$ & $\begin{array}{c}0,28(0,14) \\
0,33 \\
(0,15 ; 0,40)\end{array}$ & $\begin{array}{c}0,24(0,12) \\
0,20 \\
(0,15 ; 0,38)\end{array}$ & $0,099^{t p}$ & $0,496^{\mathrm{M}}$ & $\begin{array}{l}0,837 \\
M\end{array}$ \\
\hline Vd/Vt pico & $\begin{array}{c}0,17(0,07) \\
0,15 \\
(0,11 ; 0,22)\end{array}$ & $\begin{array}{c}0,13(0,05) \\
0,12 \\
(0,10 ; 0,17)\end{array}$ & $0,061^{w}$ & $\begin{array}{c}0,16(0,07) \\
0,16 \\
(0,10 ; 0,21)\end{array}$ & $\begin{array}{c}0,14(0,05) \\
0,12 \\
(0,10 ; 0,18)\end{array}$ & $0,023^{w}$ & $0,762^{\mathrm{M}}$ & $\begin{array}{l}0,910 \\
M\end{array}$ \\
\hline RV, \% & $\begin{array}{c}46,8(14,4) \\
47,4 \\
(35,7 ; 54,3)\end{array}$ & $\begin{array}{c}56,7(11,2) \\
55,5 \\
(48,6 ; 65,7)\end{array}$ & $0,023^{t}$ & $\begin{array}{c}49,2(16,8) \\
49,3 \\
(37,6 ; 62,5)\end{array}$ & $\begin{array}{c}51,5(14,3) \\
54,2 \\
(41,3 ; 61,2)\end{array}$ & $0,679^{t}$ & $0,580^{t}$ & $0,151^{t}$ \\
\hline $\begin{array}{l}\mathrm{T} 1 / 2_{2} \mathrm{VO}_{2} \\
\text { seg. }\end{array}$ & $\begin{array}{c}117,3(49,1) \\
102,5 \\
(85,0 ; 148,8)\end{array}$ & $\begin{array}{c}109,6(33,2) \\
110,0 \\
(85,0 ; 135,0)\end{array}$ & $0,613^{w}$ & $\begin{array}{c}102,0(41,7) \\
100,0 \\
(75,0 ; 120,0)\end{array}$ & $\begin{array}{c}105,9(30,9) \\
100,0 \\
(85,0 ; 130,0)\end{array}$ & $0,768^{w}$ & $0,200^{\mathrm{M}}$ & $\begin{array}{c}0,560 \\
M\end{array}$ \\
\hline $\begin{array}{l}\Delta \mathrm{T}^{1} 1 / 2 \mathrm{VO}_{2} \\
\%\end{array}$ & $\begin{array}{r}4,5 \\
0 \\
(-17,6\end{array}$ & $\begin{array}{l}0,0) \\
21,4)\end{array}$ & & $(-17$ & $\begin{array}{l}43,8) \\
3 \\
34,6)\end{array}$ & & & \\
\hline $\begin{array}{l}\mathrm{VE} / \mathrm{VCO}_{2} \\
\text { slope }\end{array}$ & $\begin{array}{c}38,26(12,05) \\
36,03 \\
(30,39 ; 1,01)\end{array}$ & $\begin{array}{c}32,28(4,69) \\
30,90 \\
(29,27 ; 34,96)\end{array}$ & $0,003^{w}$ & $\begin{array}{c}33,21(8,29) \\
31,70 \\
(27,08 ; 36,66)\end{array}$ & $\begin{array}{c}32,50(5,79) \\
31,16 \\
(28,87 ; 34,32)\end{array}$ & $0,870^{w}$ & $0,026^{\mathrm{M}}$ & $\begin{array}{l}0,899 \\
M\end{array}$ \\
\hline $\begin{array}{l}\Delta \mathrm{VE} / \mathrm{VCO}_{2} \\
\text { slope, } \%\end{array}$ & $\begin{array}{r}-9,9 \\
-8 \\
(-19,3\end{array}$ & $\begin{array}{l}3,6) \\
7 \\
-0,4)\end{array}$ & & 0,6 & $\begin{array}{l}8,9) \\
8,9)\end{array}$ & & & \\
\hline OUES & $\begin{array}{c}1359(556) \\
1194 \\
(1027 ; 1707)\end{array}$ & $\begin{array}{c}1294(409) \\
1164 \\
(1017 ; 1613)\end{array}$ & $0,164^{w}$ & $\begin{array}{c}1619(477) \\
1594 \\
(1268 ; 1957)\end{array}$ & $\begin{array}{c}1479(407) \\
1440 \\
(1198 ; 1743)\end{array}$ & $0,004^{w}$ & $0,07^{\mathrm{M}}$ & $\begin{array}{l}0,054 \\
M\end{array}$ \\
\hline
\end{tabular}


continuação

\begin{tabular}{|c|c|c|c|c|c|c|c|c|}
\hline \multirow{2}{*}{$\begin{array}{l}\text { TCPE } \\
\text { Bicicleta }\end{array}$} & \multicolumn{2}{|c|}{ Troca Valvar Mitral } & \multicolumn{4}{|c|}{ Reparo Mitral } & $\begin{array}{l}\text { TVM x } \\
\text { RM } \\
\text { Pré }\end{array}$ & $\begin{array}{c}\text { TVM x } \\
\text { RM } \\
\text { Pós }\end{array}$ \\
\hline & $\begin{array}{c}\text { Pré } \\
\text { Média (DP) } \\
\text { Mediana } \\
\text { (per25;per75) }\end{array}$ & $\begin{array}{c}\text { Pós } \\
\text { Média (DP) } \\
\text { Mediana } \\
\text { (per25;per75) }\end{array}$ & p & $\begin{array}{c}\text { Pré } \\
\text { Média (DP) } \\
\text { Mediana } \\
\text { (per25;per75) }\end{array}$ & $\begin{array}{c}\text { Pós } \\
\text { Média (DP) } \\
\text { Mediana } \\
\text { (per25;per75) }\end{array}$ & p & p & p \\
\hline$\Delta$ OUES, $\%$ & $\begin{array}{r}-2,4 \\
- \\
(-16\end{array}$ & $\begin{array}{l}8,2) \\
6,3)\end{array}$ & & $(-17$ & $\begin{array}{l}7,0) \\
9,7)\end{array}$ & & & \\
\hline $\begin{array}{l}\text { OUES \% } \\
\text { predito }\end{array}$ & $\begin{array}{c}62,8(15,1) \\
63,0 \\
(56,1 ; 72,3)\end{array}$ & $\begin{array}{c}61,4(11,4) \\
60,0 \\
(51,7 ; 74,3)\end{array}$ & $0,153^{t p}$ & $\begin{array}{c}68,8(12,9) \\
67,4 \\
(58,7 ; 67,4)\end{array}$ & $\begin{array}{c}62,8(10,0) \\
64,3 \\
(55,4 ; 68,8)\end{array}$ & $0,006^{\ddagger p}$ & $0,103^{t}$ & $0,597^{t}$ \\
\hline
\end{tabular}

$\mathrm{FC}$, frequência cardíaca; RER, razão de troca gasosa; $M E T$, equivalente metabólico; $\mathrm{VO}_{2}$, consumo de oxigênio; LA, limiar anaeróbio; $\mathrm{RV}$, reserva ventilatória; $\mathrm{Vd} / \mathrm{Vt}$, relação espaço morto ventilatório/volume corrente; $\mathrm{VE} / \mathrm{VCO}_{2}$ slope, inclinação do equivalente de ventilação do $\mathrm{CO}_{2}$; OUES, Oxygen Uptake Efficiency Slope; $\Delta$, diferença relativa.

Foi avaliado o efeito de interação entre tempo e grupo nas variáveis do TCPE na bicicleta que apresentaram evidências estatísticas, mesmo que fracas, entre os grupos e para pelo menos um dos tempos (Tabela 21). Os resultados mostraram não haver efeito de interação para MET pico, $\mathrm{VO}_{2}$ no limiar anaeróbico, $\mathrm{VO}_{2}$ pico e OUES. Para as três primeiras houve somente efeito significativo de tempo, e para o OUES, efeitos significativos de grupo e tempo, ou seja, o grupo TVM apresentou menores valores que o grupo RM e ambos diminuíram significativamente com o tempo e da mesma forma (Figura 10). Já para a variável $\mathrm{VE} / \mathrm{VCO}_{2}$ slope ocorreu efeito significativo da interação entre tempo e grupo. Neste caso, somente o grupo TVM reduziu o $\mathrm{VE} / \mathrm{VCO}_{2}$ slope significativamente após a cirurgia, porém antes da cirurgia o grupo TVM apresentava maiores valores. Após a cirurgia, o $\mathrm{VE} / \mathrm{VCO}_{2}$ slope se comporta de forma semelhante nos dois grupos (Figura 10). 
Tabela 21 - Valores de p da ANOVA para avaliação do efeito de interação entre tempo e grupo para as variáveis do TCPE na bicicleta

\begin{tabular}{|c|c|c|c|}
\hline & Grupo & Tempo & Grupo*Tempo \\
\hline MET pico (A np) & 0,1650 & 0,0002 & 0,3409 \\
\hline $\mathrm{VO}_{2}(\mathrm{LA}), \mathrm{mL} \mathrm{min}^{-1}(\mathrm{~A})$ & 0,2110 & 0,0200 & 0,1950 \\
\hline $\mathrm{VO}_{2}$ pico, mL.min ${ }^{-}(\mathrm{A})$ & 0,1720 & 0,0160 & 0,7320 \\
\hline VE/VCO $\mathrm{CO}_{2}$ slope (A np) & 0,1148 & 0,0177 & 0,0108 \\
\hline OUES (A np) & 0,0261 & 0,0159 & 0,3150 \\
\hline
\end{tabular}

MET, equivalente metabólico; $\mathrm{VO}_{2}$, consumo de oxigênio; LA, limiar anaeróbio; VE/ $/ \mathrm{VCO}_{2}$ slope, inclinação do equivalente de ventilação do $\mathrm{CO}_{2}$; OUES, Oxygen Uptake Efficiency Slope; A: ANOVA; A np, ANOVA não paramétrica. 

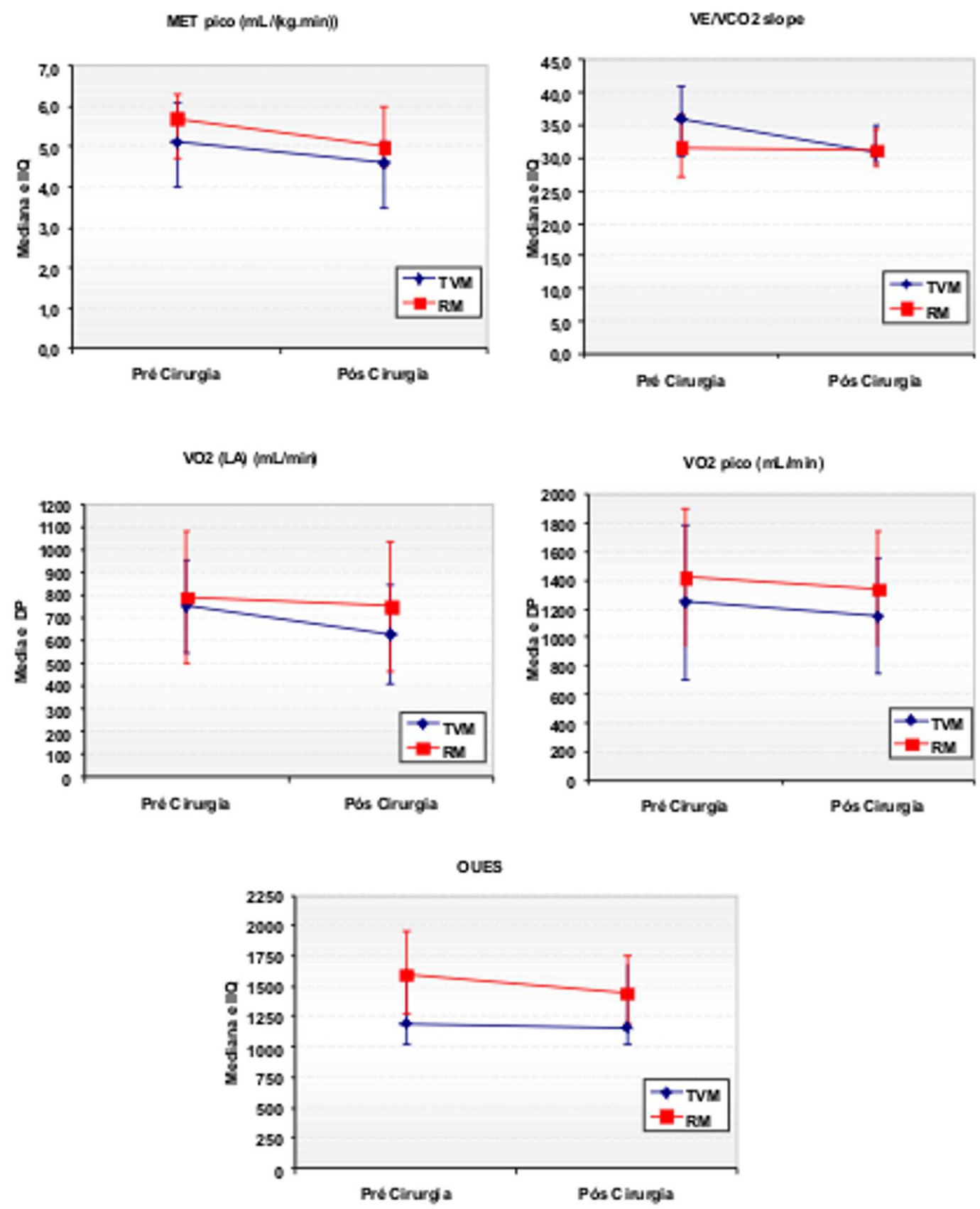

Figura 10 -Representação esquemática do efeito de interação entre tempo e grupos (TVM e RM) nas variáveis do TCPE realizado na bicicleta.

Nos TCPE realizados na esteira, a FC pico também apresentou significativa redução no momento pós-operatório, nos grupos TVM e RM, sem diferenças entre os grupos (tabela 22). 
$\mathrm{O} \mathrm{VO}_{2}$ pico evoluiu com leve redução pós-operatória em ambos os grupos, sendo que o grupo RM apresentou valor significativamente maior tanto antes quanto após a cirurgia. $\mathrm{O}$ pulso de $\mathrm{O}_{2}$ apresentou significativo aumento no momento pós-operatório somente no grupo RM e não houve diferenças pré e pós-operatórias entre os grupos na relação $\mathrm{VE} / \mathrm{VCO}_{2}$ slope. Apesar de os menores valores pré e pós-operatórios da variável OUES no grupo TVM, a porcentagem predita foi similar em ambos os grupos.

Tabela 22 - Efeito da cirurgia nas variáveis do TCPE realizados na esteira em pacientes submetidos à troca valvar mitral e reparo mitral

\begin{tabular}{|c|c|c|c|c|c|c|c|c|}
\hline \multirow[t]{2}{*}{$\begin{array}{l}\text { TCPE } \\
\text { Esteira }\end{array}$} & \multicolumn{2}{|c|}{$\begin{array}{c}\text { Troca Valvar Mitral } \\
\text { (TVM) }\end{array}$} & \multicolumn{4}{|c|}{$\begin{array}{c}\text { Reparo mitral } \\
\text { (RM) }\end{array}$} & \multirow{2}{*}{$\begin{array}{c}\text { TVM x } \\
\text { RM } \\
\text { Pré } \\
\text { Valor } \\
\text { de } \\
\text { p }\end{array}$} & \multirow{2}{*}{$\begin{array}{c}\text { TVM x } \\
\text { RM } \\
\text { Pós } \\
\text { Valor } \\
\text { de } \\
\text { p }\end{array}$} \\
\hline & $\begin{array}{c}\text { Pré } \\
\text { Média (DP) } \\
\text { Mediana } \\
\text { (per25;per75) }\end{array}$ & $\begin{array}{c}\text { Pós } \\
\text { Média (DP) } \\
\text { Mediana } \\
\text { (per25;per75) }\end{array}$ & $\begin{array}{c}\text { Valor } \\
\text { de } \\
p\end{array}$ & $\begin{array}{c}\text { Pré } \\
\text { Média (DP) } \\
\text { Mediana } \\
\text { (per25;per75) }\end{array}$ & $\begin{array}{c}\text { Pós } \\
\text { Média (DP) } \\
\text { Mediana } \\
\text { (per25;per75) }\end{array}$ & $\begin{array}{c}\text { Valor } \\
\text { de } \\
p\end{array}$ & & \\
\hline $\begin{array}{l}\text { Tempo de } \\
\text { exercício, } \\
\text { min. }\end{array}$ & $\begin{array}{c}8,0(2,8) \\
8,6 \\
(5,7 ; 10,2)\end{array}$ & $\begin{array}{c}8,6(2,5) \\
8,9 \\
(6,6 ; 10,3)\end{array}$ & $0,188 w$ & $\begin{array}{c}9,12(1,9) \\
9,7 \\
(8,2 ; 10,4)\end{array}$ & $\begin{array}{c}8,7(2,0) \\
8,8 \\
(7,6 ; 10,3)\end{array}$ & $0,079 w$ & $0,111^{\mathrm{M}}$ & $0,927^{\mathrm{M}}$ \\
\hline $\begin{array}{l}\text { FC max } \\
\text { predita, } \\
\text { bpm }\end{array}$ & $\begin{array}{c}167,7(16,1) \\
167,0 \\
(154,0 ; 181,0)\end{array}$ & $\begin{array}{c}166,0(16,7) \\
164,0 \\
(151,5 ; 180,0)\end{array}$ & 0,098 tp & $\begin{array}{c}172,2(17,4) \\
169,0 \\
(159,0 ; 188,5)\end{array}$ & $\begin{array}{c}170,9(17,6) \\
168,0 \\
(158,5 ; 187,0)\end{array}$ & 0,129 tp & $0,2871 \mathrm{t}$ & $0,2667 t$ \\
\hline $\begin{array}{l}\text { FC } \\
\text { repouso, } \\
\text { bpm }\end{array}$ & $\begin{array}{c}82,4(21,1) \\
78,0 \\
(68,5 ; 92,0)\end{array}$ & $\begin{array}{c}80,9(17,1) \\
80,0 \\
(63,5 ; 94,0)\end{array}$ & 0,824 tp & $\begin{array}{c}80,3(16,3) \\
77,0 \\
(69,0 ; 90,5)\end{array}$ & $\begin{array}{c}85,7(16,2) \\
85,0 \\
(73,5 ; 95,0)\end{array}$ & 0,063 tp & $0,792^{\mathrm{M}}$ & $0,292^{\mathrm{M}}$ \\
\hline $\begin{array}{l}\text { FC pico, } \\
\text { bpm }\end{array}$ & $\begin{array}{c}149,0(28,8) \\
151,0 \\
(128,5 ; 172,0)\end{array}$ & $\begin{array}{c}129,5(27,7) \\
130,0 \\
(110,5 ; 152,0)\end{array}$ & $0,007 t p$ & $\begin{array}{c}154,6(24,5) \\
160,0 \\
(139,5 ; 170,5)\end{array}$ & $\begin{array}{c}137,4(22,6) \\
136,0 \\
(123,0 ; 153,5)\end{array}$ & $\begin{array}{c}<0,001 t \\
p\end{array}$ & $0,489^{\mathrm{M}}$ & $0,289^{\mathrm{M}}$ \\
\hline RER pico & $\begin{array}{c}1,10(0,12) \\
1,13 \\
(1,00 ; 1,18)\end{array}$ & $\begin{array}{c}1,17(0,15) \\
1,18 \\
(1,07 ; 1,26)\end{array}$ & 0,073tp & $\begin{array}{c}1,13(0,15 \\
1,16 \\
(1.03 ; 1,24)\end{array}$ & $\begin{array}{c}1,14(0,16) \\
1,16 \\
(1.03 ; 1,28)\end{array}$ & $0,698 \mathrm{tp}$ & $0,3205 t$ & $0,5674 t$ \\
\hline MET pico & $\begin{array}{c}6,2(1,9) \\
6,0 \\
(5,0 ; 7,8)\end{array}$ & $\begin{array}{c}5,9(1,4) \\
5,6 \\
(5,2 ; 6,4)\end{array}$ & $0,256 \mathrm{~W}$ & $\begin{array}{c}6,8(1,9) \\
6,5 \\
(5,7 ; 7,8)\end{array}$ & $\begin{array}{c}6,3(1,4) \\
6,1 \\
(5,2 ; 7,3)\end{array}$ & $0,023 W$ & $0,254^{\mathrm{M}}$ & $0,168^{\mathrm{M}}$ \\
\hline $\begin{array}{l}\mathrm{VO}_{2}(\mathrm{LA}) \\
\mathrm{mL} \cdot \mathrm{min}^{-1}\end{array}$ & $\begin{array}{c}971(359) \\
909 \\
(773 ; 1086)\end{array}$ & $\begin{array}{c}950(332) \\
872 \\
(764 ; 1062)\end{array}$ & $0,681 \mathrm{~W}$ & $\begin{array}{c}1107(381) \\
1120 \\
(814 ; 1405)\end{array}$ & $\begin{array}{c}1060(309) \\
1010 \\
(799 ; 1225)\end{array}$ & $0,262 \mathrm{~W}$ & $0,132^{\mathrm{M}}$ & $0,093^{\mathrm{M}}$ \\
\hline
\end{tabular}


continuação

\begin{tabular}{|c|c|c|c|c|c|c|c|c|}
\hline \multirow[t]{2}{*}{$\begin{array}{l}\text { TCPE } \\
\text { Esteira }\end{array}$} & \multicolumn{2}{|c|}{$\begin{array}{c}\text { Troca Valvar Mitral } \\
\text { (TVM) }\end{array}$} & \multicolumn{4}{|c|}{$\begin{array}{l}\text { Reparo mitral } \\
\text { (RM) }\end{array}$} & \multirow{2}{*}{$\begin{array}{c}\text { TVM x } \\
\text { RM } \\
\text { Pré } \\
\text { Valor } \\
\text { de } \\
\text { p }\end{array}$} & \multirow{2}{*}{$\begin{array}{c}\text { TVM x } \\
\text { RM } \\
\text { Pós } \\
\text { Valor } \\
\text { de } \\
\text { p }\end{array}$} \\
\hline & $\begin{array}{c}\text { Pré } \\
\text { Média (DP } \\
\text { Mediana } \\
\text { (per25;per75) }\end{array}$ & $\begin{array}{c}\text { Pós } \\
\text { Média (DP } \\
\text { Mediana } \\
\text { (per25;per75) }\end{array}$ & $\begin{array}{l}\text { Valor } \\
\text { de } \\
p\end{array}$ & $\begin{array}{c}\text { Pré } \\
\text { Média (DP } \\
\text { Mediana } \\
\text { (per25;per75) }\end{array}$ & $\begin{array}{c}\text { Pós } \\
\text { Média (DP } \\
\text { Mediana } \\
\text { (per25;per75) }\end{array}$ & $\begin{array}{l}\text { Valor } \\
\text { de } \\
\text { p }\end{array}$ & & \\
\hline $\begin{array}{l}\mathrm{VO}_{2} \text { pico, } \\
\text { mL.min }{ }^{-1}\end{array}$ & $\begin{array}{c}1437(561) \\
1390 \\
(1068 ; 1550)\end{array}$ & $\begin{array}{c}1404(459) \\
1302 \\
(1091 ; 1574)\end{array}$ & $0,685 \mathrm{~W}$ & $\begin{array}{c}1719(571) \\
1690 \\
(1263 ; 2110)\end{array}$ & $\begin{array}{c}1609(428) \\
1635 \\
(1257 ; 2014)\end{array}$ & $0,036 W$ & $0,037^{M}$ & $0,029^{\mathrm{M}}$ \\
\hline $\begin{array}{l}\Delta \mathrm{VO}_{2} \text { pico, } \\
\%\end{array}$ & $\begin{array}{r}4,6( \\
-2 \\
(-14,2\end{array}$ & $\begin{array}{l}6,0) \\
9 \\
; 5,6)\end{array}$ & & $(-17$ & $\begin{array}{l}2,2) \\
0 \\
11,5)\end{array}$ & & & \\
\hline $\begin{array}{l}\mathrm{VO}_{2}(\mathrm{LA}), \\
\mathrm{mL} \cdot \mathrm{kg}^{-} \\
{ }^{1} \cdot \mathrm{min}^{-1}\end{array}$ & $\begin{array}{c}14,8(4,5) \\
13,6 \\
(11,6 ; 18,1)\end{array}$ & $\begin{array}{c}14,0(3,8) \\
13,5 \\
(11,4 ; 16,1)\end{array}$ & $0,329 \mathrm{~W}$ & $\begin{array}{c}15,3(4,4) \\
15,1 \\
(12,8 ; 17,1)\end{array}$ & $\begin{array}{c}14,4(3,1) \\
14,0 \\
(11,5 ; 16,7)\end{array}$ & $0,136 \mathrm{~W}$ & $0,556^{\mathrm{M}}$ & $0,408^{\mathrm{M}}$ \\
\hline $\begin{array}{l}\mathrm{VO}_{2} \text { pico, } \\
\mathrm{mL} \cdot \mathrm{mg}^{-} \\
{ }^{1} \cdot \mathrm{min}^{-1}\end{array}$ & $\begin{array}{c}21,8(6,8) \\
20,9 \\
(17,5 ; 27,3)\end{array}$ & $\begin{array}{c}20,5(4,9) \\
19,6 \\
(18,3 ; 22,2)\end{array}$ & $0,269 \mathrm{~W}$ & $\begin{array}{c}23,8(6,7) \\
23,4 \\
(20,0 ; 27,1)\end{array}$ & $\begin{array}{c}22,0(4,9) \\
21,8 \\
(18,2 ; 25,5)\end{array}$ & $0,019 W$ & $0,247^{\mathrm{M}}$ & $0,148^{\mathrm{M}}$ \\
\hline $\begin{array}{l}\mathrm{VO}_{2}(\mathrm{LA}) \% \\
\text { predito }\end{array}$ & $\begin{array}{c}47,9(12,9) \\
45,6 \\
(38,4 ; 57,3)\end{array}$ & $\begin{array}{c}46,5(12,8) \\
47,0 \\
(39,7 ; 55,4)\end{array}$ & $0,624 t p$ & $\begin{array}{c}49,1(13,2) \\
49,0 \\
(42,0 ; 57,5)\end{array}$ & $\begin{array}{c}46,3(10,0) \\
45,0 \\
(39,0 ; 51,0\end{array}$ & 0,115 tp & $0,7125 t$ & $0,9250 \mathrm{t}$ \\
\hline $\begin{array}{l}\mathrm{VO}_{2} \max \% \\
\text { predito }\end{array}$ & $\begin{array}{c}70,1(18,1) \\
69,4 \\
(55,6 ; 86,9)\end{array}$ & $\begin{array}{c}68,6(17,2) \\
66,6 \\
(59,6 ; 77,3)\end{array}$ & $0,702 \mathrm{tp}$ & $\begin{array}{c}75,5(16,9) \\
78,6 \\
(62,4 ; 87,0)\end{array}$ & $\begin{array}{c}70,1(10,8) \\
68,8 \\
(63,4 ; 76,9)\end{array}$ & $\begin{array}{c}0,017 \\
\text { tp }\end{array}$ & $0,2204 t$ & $0,6662 \mathrm{t}$ \\
\hline $\begin{array}{l}\text { Pulso } \mathrm{O}_{2} \\
\text { pico, } \\
\text { mL.bat }^{-1}\end{array}$ & $\begin{array}{c}10,1(5,1) \\
9,0 \\
(7,5 ; 11,0)\end{array}$ & $\begin{array}{c}10,9(3,2) \\
10,0 \\
(8,0 ; 14,0)\end{array}$ & $0,395 \mathrm{~W}$ & $\begin{array}{c}11,1(3,2) \\
11,0 \\
(9,0 ; 14,0)\end{array}$ & $\begin{array}{c}11,9(3,2) \\
11,0 \\
(9,5 ; 14,0)\end{array}$ & $0,003 \mathrm{~W}$ & $0,056^{\mathrm{M}}$ & $0,152^{\mathrm{M}}$ \\
\hline $\begin{array}{l}\Delta \text { Pulso } \mathrm{O}_{2} \\
\text { pico, } \%\end{array}$ & \multicolumn{2}{|c|}{$18,1(35,5)$} & & \multicolumn{2}{|c|}{$10,5(20,6)$} & & & \\
\hline $\begin{array}{l}\text { Vd/Vt } \\
\text { repouso }\end{array}$ & $\begin{array}{c}0,40(0,06) \\
0,41 \\
(0,35 ; 0,43)\end{array}$ & $\begin{array}{c}0,37(0,11) \\
0,39 \\
(0,31 ; 0,43)\end{array}$ & $0,296 \mathrm{~W}$ & $\begin{array}{c}0,38(0,06) \\
0,38 \\
(0,35 ; 0,43)\end{array}$ & $\begin{array}{c}0,36(0,10) \\
0,39 \\
(0,32 ; 0,42)\end{array}$ & $0,103 W$ & $0,357^{\mathrm{M}}$ & $0,450^{\mathrm{M}}$ \\
\hline Vd/Vt pico & $\begin{array}{c}0,20(0,06) \\
0,20 \\
(0,16 ; 0,24)\end{array}$ & $\begin{array}{c}0,18(0,06) \\
0,19 \\
(0,15 ; 0,23)\end{array}$ & $0,160 \mathrm{~W}$ & $\begin{array}{c}0,19(0,05) \\
0,17 \\
(0,15 ; 0,22)\end{array}$ & $\begin{array}{c}0,17(0,06) \\
0,18 \\
(0,13 ; 0,21)\end{array}$ & $0,078 W$ & $0,173^{\mathrm{M}}$ & $0,397^{\mathrm{M}}$ \\
\hline$R V, \%$ & $\begin{array}{c}39,1(13,7) \\
35,7 \\
(26,1 ; 53,3)\end{array}$ & $\begin{array}{c}44,7(13,4) \\
46,4 \\
(30,9 ; 55,6)\end{array}$ & $0,044 t$ & $\begin{array}{c}36,9(15,0) \\
38,8 \\
(26,9 ; 46,2)\end{array}$ & $\begin{array}{c}38,5(16,2) \\
42,0 \\
(30,5 ; 49,8)\end{array}$ & $0,801 \mathrm{t}$ & $0,5645 t$ & $0,1253 \mathrm{t}$ \\
\hline $\begin{array}{l}\mathrm{T} 1 / 2^{\mathrm{VO}_{2}} \\
\text { seg. }\end{array}$ & $\begin{array}{c}131,4(38,2) \\
120,0 \\
(100,0 ; 155,0)\end{array}$ & $\begin{array}{c}142,2(41,5) \\
130,0 \\
(120,0 ; 170,0)\end{array}$ & $0,158^{w}$ & $\begin{array}{c}118,5(43,1) \\
115,0 \\
(90,0 ; 135,0)\end{array}$ & $\begin{array}{c}129,1(32,7) \\
135,0 \\
(100,0 ; 150,0)\end{array}$ & $0,029^{w}$ & $0,130^{\mathrm{M}}$ & $0,273^{\mathrm{M}}$ \\
\hline $\begin{array}{c}\Delta \mathrm{T} 1 / 2 \mathrm{VO}_{2} \\
\%\end{array}$ & $\begin{array}{r}13,2 \\
5 \\
(-12,\end{array}$ & $\begin{array}{l}(34,7) \\
1 \\
; 36,1)\end{array}$ & & & $\begin{array}{l}30,5) \\
4 \\
28,0)\end{array}$ & & & \\
\hline
\end{tabular}


continuação

\begin{tabular}{|c|c|c|c|c|c|c|c|c|}
\hline \multirow[t]{2}{*}{$\begin{array}{l}\text { TCPE } \\
\text { Esteira }\end{array}$} & \multicolumn{2}{|c|}{$\begin{array}{l}\text { Troca Valvar Mitral } \\
\text { (TVM) }\end{array}$} & \multicolumn{4}{|c|}{$\begin{array}{l}\text { Reparo mitral } \\
\text { (RM) }\end{array}$} & $\begin{array}{l}\text { TVM x } \\
\text { RM } \\
\text { Pré }\end{array}$ & $\begin{array}{l}\text { TVM x } \\
\text { RM } \\
\text { Pós }\end{array}$ \\
\hline & $\begin{array}{c}\text { Pré } \\
\text { Média (DP) } \\
\text { Mediana } \\
\text { (per25;per75) }\end{array}$ & $\begin{array}{c}\text { Pós } \\
\text { Média (DP) } \\
\text { Mediana } \\
\text { (per25;per75) }\end{array}$ & p & $\begin{array}{c}\text { Pré } \\
\text { Média (DP) } \\
\text { Mediana } \\
\text { (per25;per75) }\end{array}$ & $\begin{array}{c}\text { Pós } \\
\text { Média (DP) } \\
\text { Mediana } \\
\text { (per25;per75) }\end{array}$ & $p$ & $p$ & $p$ \\
\hline $\begin{array}{l}\mathrm{VE} / \mathrm{VCO}_{2} \\
\text { slope, } \%\end{array}$ & $\begin{array}{c}37,43(11,36) \\
34,57 \\
(30,30 ; 42,49)\end{array}$ & $\begin{array}{c}34,73(7,24) \\
33,70 \\
(29,11 ; 40,02)\end{array}$ & $0,648^{w}$ & $\begin{array}{c}35,34(9,11) \\
33,36 \\
(29,04 ; 38,98)\end{array}$ & $\begin{array}{c}34,43(4,61) \\
33,96 \\
(30,66 ; 37,58)\end{array}$ & $0,906^{W}$ & $0,401^{\mathrm{M}}$ & $0,956^{\mathrm{M}}$ \\
\hline $\begin{array}{l}\Delta \mathrm{VE} / \mathrm{VCO}_{2} \\
\text { slope }\end{array}$ & $\begin{array}{r}-1,7 \\
-4 \\
(-12,8\end{array}$ & $\begin{array}{l}27,1) \\
4 \\
; 17,7)\end{array}$ & & $(-10$ & $\begin{array}{l}2,6) \\
14,9)\end{array}$ & & & \\
\hline OUES & $\begin{array}{c}1599(541) \\
1484 \\
(1303 ; 1788)\end{array}$ & $\begin{array}{c}1552(477) \\
1487 \\
(1215 ; 1832)\end{array}$ & $0,391 W$ & $\begin{array}{c}1857(594) \\
1814 \\
(1386 ; 2370)\end{array}$ & $\begin{array}{c}1763(514) \\
1650 \\
(1357 ; 2070)\end{array}$ & $0,073 W$ & $0,079^{\mathrm{M}}$ & $0,126^{\mathrm{M}}$ \\
\hline$\Delta$ OUES, $\%$ & & $\begin{array}{c}-1,2(27,5) \\
-5,9 \\
(-14,0 ; 9,1)\end{array}$ & & $\begin{array}{r}-2,0 \\
(-20\end{array}$ & $\begin{array}{l}7)-8,0 \\
10,2)\end{array}$ & & & \\
\hline $\begin{array}{l}\text { OUES \% } \\
\text { predito }\end{array}$ & $\begin{array}{c}77,4(17,3) \\
78,0 \\
(69,5 ; 85,7)\end{array}$ & $\begin{array}{c}73,9(13,4) \\
74,0 \\
(63,0 ; 82,6)\end{array}$ & $0,235 t$ & $\begin{array}{c}78,6(15,5) \\
76,8 \\
(68,6 ; 89,5)\end{array}$ & $\begin{array}{c}74,7(14,6) \\
74,2 \\
(65,6 ; 80,8)\end{array}$ & $0,108 t$ & $0,773 t$ & $0,818 t$ \\
\hline
\end{tabular}

FC, frequência cardíaca; RER, razão de troca gasosa; MET, equivalente metabólico; $\mathrm{VO}_{2}$, consumo de oxigênio; LA, limiar anaeróbio; $R V$, reserva ventilatória; $V d / V t$, relação espaço morto ventilatório/volume corrente; $\mathrm{VEN} / \mathrm{VCO}_{2}$ slope, inclinação do equivalente de ventilação do $\mathrm{CO}_{2}$; OUES, Oxygen Uptake Efficiency Slope; $\Delta$, diferença relativa.

Não houve efeito significativo de interação entre tempo e grupo no $\mathrm{VO}_{2}$ pico, Pulso de $\mathrm{O}_{2}$ pico e OUES, ou seja, os grupos não se comportam diferentes com o tempo. Nestes casos ocorreram efeitos somente de grupo em $\mathrm{VO}_{2}$ pico, de tempo e grupo no Pulso de $\mathrm{O}_{2}$ pico e de grupo no OUES (Tabela 23 e Figura 11). 
Tabela 23 - Valores de $p$ da ANOVA para avaliação do efeito de interação entre tempo e grupo para as variáveis do TCPE na esteira

\begin{tabular}{lccc}
\hline & Grupo & Tempo & Grupo ${ }^{\star}$ Tempo \\
\hline VO $_{2}$ pico, ${\mathbf{~ m L} \cdot \mathrm{min}^{-1} \text { (A np) }}^{-1}$ & $\mathbf{0 , 0 2 4 5}$ & 0,1318 & 0,8016 \\
Pulso $\mathrm{O}_{2}$ pico, mL.bat $^{-1}$ (A np) & $\mathbf{0 , 0 7 1 1}$ & $\mathbf{0 , 0 0 2 0}$ & 0,4661 \\
OUES (A np) & $\mathbf{0 , 0 5 5 4}$ & 0,2775 & 0,6357 \\
\hline
\end{tabular}

$\mathrm{VO}_{2}$, consumo de oxigênio; OUES, Oxygen Uptake Efficiency Slope; A, ANOVA; A np, ANOVA não paramétrica.
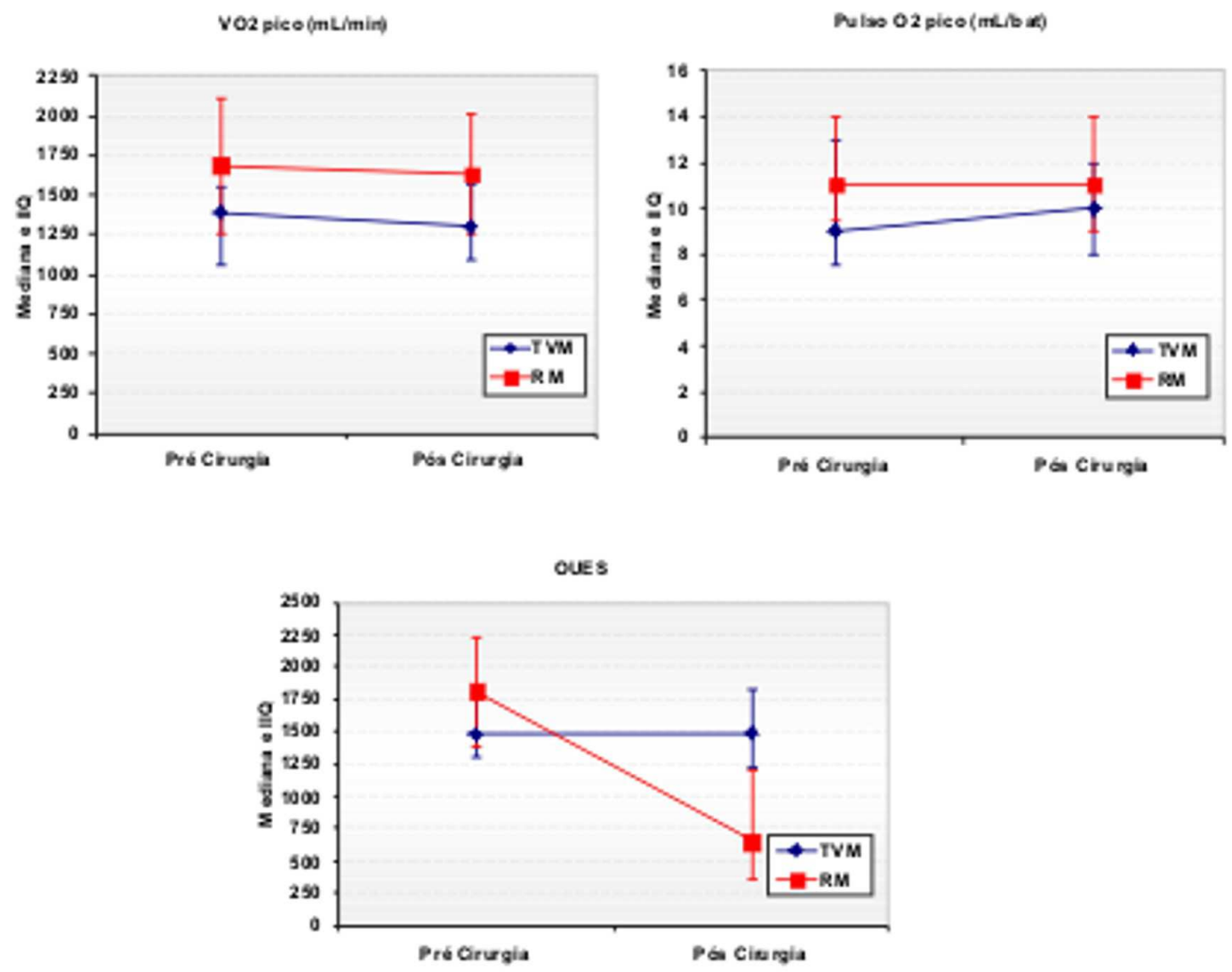

Figura 11 - Representação esquemática do efeito de interação entre tempo e grupos (TVM e RM) nas variáveis do TCPE realizado na esteira. 
Discussão 


\subsection{Considerações preliminares}

A IM crônica apresenta patofisiologia complexa e impõe sobrecarga volumétrica ao VE, podendo levar à queda irreversível de sua contratilidade. As condições favoráveis de carga imposta ao VE durante longo período de sua história natural mantêm o paciente com poucos sintomas ou assintomático, mesmo na presença de disfunção contrátil do $\mathrm{VE}^{18,19}$.

A avaliação da função contrátil ventricular esquerda pelos métodos habitualmente disponíveis, em geral derivados do ecocardiograma como, por exemplo, a FE, pode mostrar-se inadequada e por vezes não refletindo o real estado da contratilidade do $\mathrm{VE}^{23}$.

O tratamento definitivo da IM crônica e grave é o cirúrgico e inclui a troca valvar mitral e o reparo mitral. Os melhores resultados cirúrgicos imediatos e tardios do reparo mitral em relação à troca valvar mitral devemse basicamente a manutenção do aparato subvalvar mitral, preservando a geometria e função ventricular esquerda, além da ausência de complicações relacionadas às próteses biológicas ou mecânicas ${ }^{30}$.

As diretrizes atuais da $\mathrm{ACC} / \mathrm{AHA}^{45}$ recomendam a intervenção cirúrgica na IM grave e crônica na presença de sintomas de insuficiência cardíaca ou se houver evidências de disfunção ventricular esquerda, HAP ou 
aparecimento de fibrilação atrial. No paciente com IM assintomática, as mesmas diretrizes recomendam cirurgia precoce se houver alta probabilidade de reparo mitral com sucesso, porém esta estratégia não é universalmente aceita ${ }^{12}$. Em estudo de Rosenhek et al. ${ }^{11}$, os pacientes com IM assintomática foram seguidos clinicamente com bons resultados até 0 início dos sintomas ou de evidências de disfunção ventricular esquerda, HAP ou aparecimento de fibrilação atrial. Portanto, permanece controversa a indicação cirúrgica no paciente com IM e assintomático.

Com o objetivo de não alterar a rotina de acompanhamento pósoperatória dos pacientes submetidos à cirurgia de troca valvar mitral ou reparo em nosso serviço, no nosso estudo os pacientes não foram submetidos a programas de treinamento ou reabilitação. Poucos trabalhos têm avaliado a capacidade de exercício após a cirurgia cardíaca, particularmente no paciente portador de doença valvar ${ }^{59,61,62}$. No estudo de Le Tourneau et $a \beta^{\beta 7}$, não houve melhora da tolerância ao exercício após a cirurgia de correção da IM em pacientes não treinados. O autor considera que estes resultados poderiam ser justificados pelo próprio descondicionamento físico induzido pela valvopatia ou pelo curso pósoperatório.

Além disso, no período pós-operatório precoce de uma cirurgia cardíaca, muitos fatores, podem contribuir para uma redução da capacidade de exercício em relação ao nível pré-operatório, dentre eles podemos citar: piora ventilatória (por derrame pleural, atelectasia ou paralisia do nervo frênico), IC congestiva, anemia, mobilidade diminuída das costelas e do 
esterno, taquicardia sinusal, FA, disfunção ventricular esquerda transitória e fadiga global ${ }^{69-71}$.

As limitações e as dificuldades de se quantificar a classe funcional de forma adequada, principalmente no indivíduo sedentário, sugerem a necessidade de uma avaliação precisa e objetiva ${ }^{72}$. O TCPE preenche esta lacuna, pois fornece de forma quantitativa, objetiva e não invasiva a CF, além de inúmeras variáveis para estratificação prognóstica ${ }^{73}$.

$\mathrm{Na}$ insuficiência mitral crônica, pouco se conhece sobre 0 comportamento do TCPE ao longo de sua história natural e após a correção cirúrgica. Isso se deve aos poucos trabalhos realizados, com limitado número de pacientes e não randomizados. No estudo de Messika-Zeitoun et al. ${ }^{80}$, em 134 pacientes com IM orgânica e assintomática, o TCPE contribuiu para revelar limitação funcional não detectada clinicamente, sendo considerada, portanto, importante ferramenta no manejo desses pacientes.

Pelo nosso conhecimento, o presente estudo é o primeiro a avaliar prospectivamente os efeitos da cirurgia valvar em pacientes com IM isolada, orgânica, grave e não isquêmica, nas variáveis do TCPE realizados em esteira rolante e bicicleta ergométrica.

Existem diferenças na resposta orgânica durante o exercício realizado na esteira ou na bicicleta ergométrica. Habitualmente, os testes em bicicleta são interrompidos por fadiga de musculatura dos membros inferiores e o $\mathrm{VO}_{2}$ é em média 10 a $20 \%$ abaixo daquele obtido com o exercício em esteira ergométrica ${ }^{66}$. Estas diferenças no $\mathrm{VO}_{2}$ são justificadas pelos maiores valores no débito cardíaco e diferença arteriovenosa de oxigênio quando o 
exercício é realizado na esteira. Entretanto, a execução do exercício em bicicleta apresenta menor risco de queda, facilidade dos registros eletrocardiográficos e de pressão arterial devido à menor movimentação do tronco e apresenta como principal vantagem a possibilidade da mensuração precisa da relação potência aplicada e demanda metabólica.

No entanto, como não dispomos na literatura de informações definitivas sobre as possíveis diferenças na resposta aos exercícios realizados na bicicleta e na esteira, em pacientes portadores de IM e submetidos à correção cirúrgica, optamos por incluir e analisar as duas modalidades de esforço em nosso estudo.

Os resultados pré e pós-operatórios das variáveis do TCPE foram comparados, sendo que o TCPE pós-operatório foi realizado após um período de progressiva e significativa remodelamento cardíaca, conforme comprovado pelo estudo ecocardiográfico realizado em média oito meses após a cirurgia valvar.

O objetivo principal deste estudo foi avaliar os efeitos da correção cirúrgica nas variáveis do TCPE em pacientes com IM crônica e isolada. Além disso, procuramos identificar quais variáveis do TCPE poderiam estar associadas com os parâmetros ecocardiográficos de remodelamento cardíaca. Realizamos também uma avaliação comparativa do TCPE nos pacientes submetidos à troca valvar mitral e reparo mitral.

As comparações entre o TCPE realizado na esteira e na bicicleta não foram objeto de análise no estudo atual, porém um quadro comparativo tanto 
no pré como no pós-operatório estão inseridos nos anexos 7 e 8, respectivamente.

Em nossa casuística, houve predominância do sexo masculino em 41 pacientes $(56,9 \%)$, da etiologia degenerativa em 50 pacientes $(69,4 \%)$ e da classe funcional I ou II pela NYHA em 51 pacientes (70,8\%). Oito pacientes $(11,1 \%)$ apresentavam fibrilação atrial permanente. A cirurgia mais frequentemente realizada foi o reparo mitral em 47 pacientes $(65,3 \%)$.

Não houve diferenças significativas nas características de base nos grupos troca valvar mitral e reparo mitral. O grupo RM apresentou predominância do sexo masculino e a média das idades não apresentou diferença estatística com o grupo troca valvar. Também não houve diferença estatística em ambos os grupos na proporção de pacientes com a etiologia degenerativa e reumática.

Após pouco mais de 7 meses da cirurgia corretiva da IM, houve um aumento significativo do IMC, com ganho médio de $1,42 \mathrm{Kg}$ de peso corporal. Acreditamos que este aumento de peso esteja relacionado ao sedentarismo comumente observado após a cirurgia cardíaca, já que houve melhora da CF pela NHYA e consistente remodelamento positiva do ventrículo esquerdo. Também não foram identificadas situações clínicas que pudessem limitar a atividade física no pós-operatório. Além disso, a prova de função pulmonar realizada junto com os TCPE apresentou valores normais e acima de $90 \%$ do predito para a CVF, VEF1 e VVM tanto antes quanto após a cirurgia, não havendo, portanto, comprometimento pulmonar prévio ou relacionado ao procedimento cirúrgico. 
Os pacientes relataram melhora dos sintomas após a cirurgia, apesar do ganho de peso, acima relatado. A classe funcional avaliada pela NYHA apresentou após a cirurgia redução significativa de em média 2,06 $\pm 0,80$ para $1,17 \pm 0,38$. No momento pós-operatório, houve redução do número de pacientes com FA ( $p=N S)$ e maior proporção de pacientes recebendo medicação beta-bloqueadora ( $p=N S)$ e amiodarona $(p=0,014)$. A diferença estatisticamente significativa, observada nos valores pré e pós-operatórios da glicemia, uréia e creatinina, não apresentam significado clinico por se manterem dentro da faixa de normalidade.

Como poderia se esperar, o ecocardiograma realizado em média 7,3 meses após a cirurgia revelou expressiva redução dos volumes atrial e ventricular esquerdo, além de redução da pressão em artéria pulmonar. As reduções, avaliada pela diferença relativa, foram de $-36,0 \pm 19,7 \%$, $-27,4 \pm 20,1 \%,-7,1 \pm 32,1 \%$ e $-22,6 \pm 28,5 \%$, para o VAE, VDFVE, VSFVE e PSAP respectivamente.

Diferentemente do que é observado na literatura, em que o reparo mitral está associado à melhor função ventricular pós-operatória quando comparado aos pacientes submetidos à troca valvar, no estudo atual não houve diferenças significativas na remodelamento ventricular esquerda em ambos os grupos.

A fração de ejeção apresentou redução significativa após a cirurgia nos grupos TVM e RM, com uma diferença relativa de $-9,5 \pm 11,3 \%$ no grupo TVM e de $-11,7 \pm 12,0 \%$ no grupo RM, mantendo-se dentro da normalidade no momento pós-operatório para os dois grupos. Além disso, a fração de 
ejeção não apresentou diferenças entre os grupos, tanto no pré como pósoperatório. Os volumes ventriculares, sobretudo o diastólico, também apresentaram reduções significativas no momento pós-operatório nos grupos troca valvar mitral e reparo mitral. Estas observações sugerem que os pacientes envolvidos no estudo tiveram sua indicação e intervenção cirúrgica realizada antes do início de disfunção ventricular esquerda irreversível, independente do tipo de cirurgia realizada.

Apesar da significativa redução do VAE em ambos os grupos, os pacientes do grupo RM apresentaram menor VAE em relação ao grupo TVM, mais evidente no pós-operatório. Este achado sugere que o momento de indicação cirúrgica nos pacientes que realizaram o reparo mitral foi mais precoce em relação aos pacientes que realizaram a troca valvar mitral. Estudos prévios sugerem que o tamanho do AE, além de ser um marcador prognóstico, é uma informação importante por refletir a duração e gravidade da insuficiência mitral crônica ${ }^{77}$.

\subsection{Teste cardiopulmonar de exercício realizado na bicicleta}

Em nossa casuística, os TCPE pré-operatório na bicicleta foram realizados em média menos de um mês antes da cirurgia e os pósoperatórios após 7 meses da correção cirúrgica da IM. Apesar de o tempo de exercício ter sido similar antes e após a cirurgia, houve reduções significativas na $\mathrm{FC}$ pico, MET pico e $\mathrm{VO}_{2}$ pico. 
No pico do esforço a FC diminuiu significativamente de $144,3 \pm 27,2$ bat. $\mathrm{min}^{-1}$ no pré-operatório para $126,5 \pm 24,6$ bat. $\mathrm{min}^{-1}$ no pósoperatório $(p<0,0001)$ e justificado pelo maior número de pacientes recebendo medicação beta-bloqueadora e amiodarona após a cirurgia. Antes da cirurgia 30 pacientes $(43,5 \%)$ e após a cirurgia 38 pacientes $(54,3 \%)$ estavam recebendo beta-bloqueador ( $p=N S$ ) na ocasião da realização do TCPE. O número de pacientes que estavam recebendo amiodarona aumentou de quatro pacientes $(5,8 \%)$ no pré-operatório para 14 pacientes $(20,0 \%)$ no pós-operatório $(p=0,016)$. Convém salientar que a prescrição dos medicamentos foi definida pelo médico assistente conforme a rotina do serviço, e os TCPE foram realizados sem a suspensão das medicações em uso.

Apesar de o uso de medicações específicas, em ambos os momentos pré e pós-operatório a qualidade ou intensidade do esforço foi considerada satisfatória, como demonstrada pela relação de troca gasosa (RER) $>1,10$ e, portanto, preenchido os critérios de maximalidade do esforço.

Após a cirurgia não houve diferença na carga alcançada, porém o $\mathrm{VO}_{2}$ pico diminuiu após a cirurgia, com uma diferença relativa de $-3,0 \pm 20,2 \%$. A porcentagem alcançada do $\mathrm{VO}_{2}$ máx predito diminuiu de $64,6 \pm 15,4 \%$ no pré-operatório para $60,6 \pm 10,8 \%$ no pós-operatório $(0,016)$.

O $\mathrm{VO}_{2}$ máx é uma importante variável do TCPE por ser considerada a métrica que define o limite do sistema cardiopulmonar ${ }^{75}$ e representa o nível máximo do metabolismo oxidativo envolvendo grandes grupos musculares ${ }^{78}$. 
Esta variável, de acordo com a equação de Fick, trata-se do produto do débito cardíaco e da diferença arteriovenosa de oxigênio (Dif. a-v $\mathrm{O}_{2}$ ). Os principais determinantes do $\mathrm{VO}_{2}$ máx são fatores genéticos e a quantidade de musculatura envolvida no exercício, e também depende do sexo, idade e superfície corporal, além do nível de treinamento ou condicionamento físico ${ }^{69} . \mathrm{OVO}_{2}$ máx é considerado reduzido quando abaixo de $80 \%$ do valor predito.

Embora tenha havido variação individual em nosso estudo, o não aumento do $\mathrm{VO}_{2}$ pico ou da porcentagem predita do $\mathrm{VO}_{2}$ contradiz com a melhora significativa da CF pela NYHA. Este achado já foi observado em poucos trabalhos prévios realizados em pacientes com IM submetidos à cirurgia $^{37,38}$. Como citado previamente, os pacientes do nosso estudo não participaram de programas de reabilitação e condicionamento físico e acreditamos que este fato possa ter contribuído para a redução do $\mathrm{VO}_{2}$ máx após a cirurgia.

$\mathrm{O}$ pulso de $\mathrm{O}_{2}$ fornece uma estimativa do volume sistólico do VE e reflete a quantidade de $\mathrm{O}_{2}$ transportada e consumida pelo organismo em cada batimento cardíaco. É considerado um forte preditor de mortalidade em pacientes com doença cardiovascular ${ }^{83}$. Em nossa casuística, o pulso de $\mathrm{O}_{2}$ pico apresentou um leve aumento após a cirurgia, de 9,5 $\pm 3,4$ para $10,1 \pm 3,1 \mathrm{~mL}$. bat ${ }^{-1}(p=0,09)$, sugerindo melhora da performance do ventrículo esquerdo. $\mathrm{O}$ comportamento ou a curva do pulso de $\mathrm{O}_{2}$ durante o esforço foi considerada normal em aproximadamente $80 \%$ dos pacientes tanto antes como após a cirurgia e uma resposta anormal (padrão de platô 
ou queda) foi observada em $16,7 \%$ dos pacientes no pré-operatório e em 18,0\% no pós-operatório, sugerindo redução do desempenho ventricular. Não houve diferenças pré e pós-operatória quando comparada as proporções de respostas do pulso de $\mathrm{O}_{2}$ durante o exercício. De acordo com - nosso conhecimento este é o primeiro trabalho que avalia o comportamento pré e pós-operatório da curva do pulso de $\mathrm{O}_{2}$ durante $\mathrm{o}$ esforço em pacientes com IM e não apenas o valor pico atingido.

$\mathrm{O}$ tempo para que o $\mathrm{VO}_{2}$ atingisse metade do seu valor máximo no esforço, na fase de recuperação ou $T_{1 / 2}$, fornece uma informação sobre a cinética do $\mathrm{O}_{2}$ após a interrupção de um esforço incremental. Em indivíduos normais os valores do $T_{1 / 2}$ apresentam valores entre 60 e 90 seg. e acima de 120 seg. é indicativo de elevada mortalidade em pacientes com insuficiência cardíaca $^{84}$. Na nossa amostragem não houve diferença do $T_{1 / 2}$ após a cirurgia, mantendo-se em 107 segundos.

Após a cirurgia, a relação $\mathrm{VE} / \mathrm{VCO}_{2}$ slope diminuiu de $34,94 \pm 9,95$ para 32,42 $\pm 5,40(p=0,078)$. Esta relação descreve a eficiência ventilatória durante o esforço, mostrando a quantidade de ar que deve ser ventilada para eliminar $1 \mathrm{~L}$ de $\mathrm{CO}_{2}$ e os valores normais se situam entre 20,00 e 30,00(66). No estudo de Arena et al.(76) uma relação $\mathrm{VE} / \mathrm{VCO}_{2}$ slope superior a 34,00 foi um marcador prognóstico desfavorável em pacientes com IC e disfunção ventricular esquerda. Não encontramos na literatura informações sobre o comportamento desta variável em pacientes com IM submetidos à correção cirúrgica, porém os resultados de nossa casuística, 
apesar da fraca evidência estatística, revelam uma melhora desta variável, com valores pós-operatórios abaixo do limiar de mau prognóstico.

A variável OUES diminuiu após a cirurgia de 1530,0 $\pm 516,3$ ( $66,7 \pm 13,9 \%$ do predito) para $1413,8 \pm 414,6$ ( $62,3 \pm 10,4 \%$ do predito) com uma diferença relativa de $-4,7 \pm 17,3 \%$. Não encontramos na literatura 0 comportamento desta variável após a cirurgia cardíaca em pacientes com IM, entretanto, uma redução do OUES foi observada em pacientes com doença arterial coronária, submetidos à cirurgia de revascularização miocárdica, quando comparados aos pacientes submetidos à angioplastia transluminal percutânea, assim como houve uma melhora significativa desta variável após treinamento físico ${ }^{86}$. A melhora do OUES também tem sido observada após treinamento físico em pacientes com $\mathrm{IC}^{87}$, sugerindo que um determinado $\mathrm{VO}_{2}$ seja alcançado com um custo ventilatório menor. Estudos prévios sugerem que o OUES é fortemente correlacionado com o $\mathrm{VO}_{2}$ pico $^{88,89}$. Em nosso estudo, é provável que a redução do OUES e do $\mathrm{VO}_{2}$ observado sete meses após a cirurgia tenha ocorrido pelo descondicionamento físico e falta de treinamento dos pacientes em programas de reabilitação.

A curva da relação $\mathrm{VO}_{2}$ /carga durante o esforço foi avaliada e comparada nos momentos pré e pós-operatório, sendo um importante marcador de disfunção ventricular esquerda e reserva cardíaca diminuída. Não houve diferença nos padrões de resposta, com a maioria dos pacientes apresentando resposta normal $(96,7 \%$ dos pacientes no pré, e, $95,1 \%$ no pós-operatório). Somente dois pacientes $(3,3 \%)$ no pré-operatório e dois 
$(3,3 \%)$ no pós-operatório apresentaram resposta anormal (padrão de queda).

Em nosso estudo, procuramos avaliar as possíveis associações de algumas variáveis do TCPE com os parâmetros ecocardiográficos de remodelamento cardíaca após a cirurgia. Observamos correlação negativa entre a medida da diferença relativa do OUES e a diferença relativa da PSAP $(r=-0,359 ; p=0,010)$. Portanto, concluímos que quanto maior a redução pós-operatória da PSAP maior a probabilidade de observarmos um aumento do OUES. Em nossa casuística a PSAP média no pré-operatório foi de 46,7 $\pm 15,9$ mm Hg na avaliação ecocardiográfica e de $39,0 \pm 13,7 \mathrm{~mm} \mathrm{Hg}$ pelo cateterismo cardíaco. Pelas diretrizes atuais da $\mathrm{ACC} / \mathrm{AHA}^{45}$, recomenda-se a cirurgia em pacientes com IM crônica, quando a PSAP é maior do que $50 \mathrm{~mm} \mathrm{Hg}$ em repouso. Não encontramos na literatura a correlação do OUES com hipertensão arterial pulmonar em pacientes com insuficiência mitral. O TCPE ainda é considerado uma ferramenta promissora em pacientes com hipertensão arterial pulmonar e as evidências atuais sugerem que possa fornecer informações prognósticas e avaliar a resposta ao tratamento ${ }^{90}$.

Nos TCPE realizados na bicicleta, a diferença percentual do pulso de $\mathrm{O}_{2}$ apresentou correlação positiva com a diferença percentual da fração de ejeção. A fração de ejeção, após a correção cirúrgica da valvopatia fornece uma avaliação mais adequada da função sistólica do ventrículo esquerdo. Portanto, essa correlação sugere que os pacientes que evoluem no pós- 
operatório com aumento do pulso de $\mathrm{O}_{2}$, provavelmente foram aqueles que aumentaram ou não apresentaram queda da fração de ejeção.

A redução do $\mathrm{VO}_{2}$ pico após a cirurgia foi observada em ambos nos grupos TVM e RM, porém mais evidente e com redução significativa apenas no grupo RM (de $1245 \pm 53$ para $1145 \pm 402 \mathrm{~mL} \cdot \mathrm{min}^{-1} 1, \mathrm{p}=0,035$ ). $\mathrm{O} \mathrm{VO} \mathrm{V}_{2}$ pico pós-operatório foi significativamente maior no grupo RM (1335 $\pm 400 \mathrm{~mL}$. $\mathrm{min}^{-}$ 1 vs $\left.1145 \pm 402 \mathrm{~mL} \cdot \min ^{-1}, \mathrm{p}=0,038\right)$. Essa observação adiciona mais uma vantagem do reparo valvar em relação à troca valvar mitral.

O pulso de $\mathrm{O}_{2}$ apresentou um aumento significativo no momento pósoperatório somente no grupo reparo mitral, de 9,8 \pm 3,0 para $10,5 \pm 3,2 \mathrm{~mL}$ bat $^{-1}(\mathrm{p}=0,033)$. Não houve diferenças nos valores pré e pósoperatórios do pulso de $\mathrm{O}_{2}$ entre os grupos troca valvar mitral e reparo mitral.

Observamos que os pacientes que realizaram o reparo mitral apresentavam maiores valores do OUES em relação ao grupo troca valvar mitral, tanto no pré como no pós-operatório. A redução pós-operatória do OUES, observada nos dois grupos, foi mais evidente no grupo reparo mitral, com diferença relativa de $-5,8 \pm 17,0 \%$. O maior valor do $\mathrm{VO}_{2}$ pico e do OUES após a cirurgia nos pacientes que realizaram o reparo mitral, incorporam na literatura, vantagens em relação à troca valvar mitral. 


\subsection{Teste cardiopulmonar de exercício realizado na esteira}

Os resultados pré e pós-operatórios dos parâmetros do TCPE realizados na esteira rolante foram avaliados e comparados. O TCPE na esteira foi realizado em média $31,6 \pm 29,4$ dias antes da cirurgia corretiva da valvopatia e 219,1 $\pm 38,3$ dias após a cirurgia.

A significativa redução da FC pico após a cirurgia, assim como o observado nos pacientes que realizaram o TCPE realizado na bicicleta, é justificado pelo maior número de pacientes recebendo medicação betabloqueadora e amiodarona no pós-operatório.

O tempo de exercício foi similar antes e após a cirurgia e a qualidade do esforço foi considerada satisfatória em ambos os momentos, com a razão $\operatorname{RER}>1,10$.

Do mesmo modo que o TCPE realizado na bicicleta e o observado na literatura, houve uma leve redução do $\mathrm{VO}_{2}$ pico, de $1618 \pm 579$ para $1536 \pm 447 \mathrm{~mL} \cdot \mathrm{min}^{-1}(\mathrm{p}=0,06)$.

$\mathrm{O}$ pulso de $\mathrm{O}_{2}$ pico apresentou aumento significativo no momento pós-operatório, de 10,7 \pm 4,0 para 11,5 $\pm 3,2{\mathrm{~mL} \cdot \text { bat }^{-1}}^{(}(\mathrm{p}=0,023$ ), porém $46,4 \%$ dos pacientes antes e $52,2 \%$ dos pacientes após a cirurgia evoluíram com curva anormal ao esforço, refletindo diminuição do desempenho sistólico do ventrículo esquerdo durante o esforço. As proporções do tipo de resposta da curva do pulso de $\mathrm{O}_{2}$ não apresentaram diferença significativa nos momentos pré e pós-operatório. 
A variável ventilatória $\mathrm{VE} / \mathrm{VCO}_{2}$ slope apresentou leve redução após a cirurgia ( $p=N S$ ) com valor pós-operatório de $34,5 \pm 5,6$ e pouco acima do limiar de mau prognóstico.

O OUES evoluiu com significativa redução após a cirurgia, de $1761 \pm 585$ para $1691 \pm 508(p=0,050)$, porem a diferença relativa foi de apenas $-1,7 \pm 25,6 \%$.

Do mesmo modo que no TCPE realizado na bicicleta, procuramos avaliar as possíveis correlações das variáveis do TCPE com as medidas ecocardiográficas de remodelamento cardíaca pós-operatória. Analisamos as correlações das diferenças relativas do $\mathrm{VO}_{2}$ pico, pulso de $\mathrm{O}_{2}$ pico, $\mathrm{T}^{1}$ 1/2 $\mathrm{VO}_{2}, \mathrm{VE} / \mathrm{VCO}_{2}$ slope e OUES com as diferenças relativas do VDFVE, VSFVE, FE, VAE e PSAP.

$\mathrm{O} \mathrm{VO}_{2}$ pico e o OUES apresentaram significativa correlação negativa com a PSAP, ou seja, quanto maior a redução da PSAP maior a probabilidade de observarmos aumento do $\mathrm{VO}_{2}$ pico e do OUES.

A variável OUES também apresentou correlação negativa com o VDFVE e o VAE.

O TCPE realizado na esteira também revelou algumas alterações nos pacientes que realizaram a troca valvar mitral e o reparo mitral.

$\mathrm{O} \mathrm{VO}_{2}$ pico mostrou com leve redução após a cirurgia, sem significado estatístico, nos pacientes que realizaram a troca valvar mitral (de $1437 \pm 561$ para $\left.1404 \pm 459 \mathrm{~mL} \cdot \mathrm{min}^{-1}, \mathrm{p}=0,685\right)$. Já no grupo reparo mitral, houve diminuição significatica do $\mathrm{VO}_{2}$ pico de $1719 \pm 571$ para $1609 \pm 428 \mathrm{~mL} \cdot \mathrm{min}^{-1}, \mathrm{p}=0,036$. 
A média dos valores do $\mathrm{VO}_{2}$ pico foi significativamente maior no grupo reparo mitral em relação à troca valvar mitral, em ambos os momentos pré ( $p=0,037)$ e pós-operatório $(p=0,029)$.

O pulso de $\mathrm{O}_{2}$ aumentou no pós-operatório em ambos os grupos, sendo que no grupo RM, o aumento foi significativo, de 11,1 $\pm 3,2$ para $11,9 \pm 3,2 \mathrm{~mL}$ bat $^{-1}(p=0,003)$, sugerindo melhor desempenho ventricular após a correção cirúrgica da valvopatia.

Não houve diferenças significativas no $\mathrm{VE} / \mathrm{VCO}_{2}$ slope e no OUES nos pacientes que realizaram a troca valvar mitral e o reparo mitral, tanto na avaliação pré quanto pós-operatória.

\subsection{Considerações finais}

Neste estudo podemos observar, em uma visão global, que algumas variáveis do TCPE de valor prognóstico, não se modificaram de forma significativa após a correção cirúrgica da insuficiência mitral crônica. O consumo pico de $\mathrm{O}_{2}$ e o OUES apresentou leve redução pós-operatória tanto no TCPE realizado na bicicleta quanto na esteira.

Uma explicação para estes "paradoxos" pode ser o descondicionamento periférico induzido tanto pela doença valvar como pelo curso pós-operatório. O treinamento físico permite melhorar a performance de exercício em pacientes portadores de doença coronária ${ }^{91}$ e $I^{92-94}$ ou mesmo após a cirurgia de revascularização miocárdica(86-88) e troca valvar $^{98}$. Os benefícios dos programas de recondicionamento físico após a 
cirurgia também foram observados em pacientes que realizaram o reparo mitral, com aumento significativo do $\mathrm{VO}_{2}$ pico de $22 \%$ e do limiar anaeróbio de $16 \%$, independente da idade, sexo, função ventricular esquerda, presença de FA, concentração de hemoglobina ou uso de medicação $(\beta \text {-bloqueador ou inibidor de enzima de conversão da angiotensina })^{99}$.

Contudo, das variáveis estudadas, $\mathrm{V}_{\mathrm{VO}_{2}}$ pico e o OUES se correlacionaram com alguns dos parâmetros ecocardiográficos de remodelamento cardíaca e de maneira inversa, nos TCPE realizados na esteira. O modelo estatístico proposto para as associações, de correlação da diferença percentual, permitiu concluir que quanto maior a redução da PSAP após a cirurgia, maior a probabilidade de observarmos aumento do $\mathrm{VO}_{2}$ pico e do OUES. De forma similar, a redução pós-operatória dos VDFVE, VAE e PSAP se associaram com um aumento do OUES.

Nos TCPE realizados na bicicleta, também identificamos uma correlação negativa do pulso de $\mathrm{O}_{2}$ com a $\mathrm{FE}$ e o OUES com a pressão sistólica em artéria pulmonar.

Estas associações indicam a importância da remodelamento cardíaca positiva após a cirurgia de correção em pacientes com IM crônica, grave e orgânica. Esses resultados incorporam informações na literatura que demonstram os benefícios da indicação cirúrgica em momento adequado, antes do aparecimento de disfunção irreversível do VE, aumento acentuado do $A E$ ou presença de hipertensão arterial pulmonar.

Os resultados observados no nosso estudo, junto com as dados obtidos da literatura existente, reforça a necessidade dos pacientes 
realizarem reabilitação física após a correção cirúrgica da valva mitral. Os benefícios da remodelamento cardíaca positiva após a cirurgia, aliado a um programa de recondicionamento físico, permitiria uma abordagem mais adequada e completa para os pacientes no seu curso pós-operatório.

O TCPE surge como método útil e promissor em pacientes com IM crônica, grave e não isquêmica, podendo auxiliar no manejo clínico e indicação cirúrgica, inclusive no paciente assintomático.

\subsection{Limitações do estudo}

Uma das limitações deste estudo foi à falta de um grupo controle, entretanto, o objetivo principal foi à avaliação do impacto da cirurgia nas variáveis do TCPE.

Outra limitação refere-se à falta de randomização entre os pacientes submetidos à troca valvar mitral e reparo mitral. O reparo tem sido a cirurgia de escolha devido aos seus benefícios e vantagens já bem estabelecidos. Portanto qualquer trabalho que envolva uma randomização entre troca valvar e reparo mitral para pacientes com IM poderia envolver questionamentos éticos importantes. Em nossa casuística houve predominância do reparo mitral, porém as características de base não diferiram de forma significativa em ambos os grupos, permitindo um quadro comparativo confiável.

Como não houve melhora de algumas variáveis do TCPE após a cirurgia mitral, é possível que isso possa ter sido influenciado pelo período 
relativamente curto de seguimento pós-operatório. Entretanto, este período foi suficiente para uma significativa remodelamento cardíaca conforme evidenciado pelas medidas ecocardiográficas. 
Conclusões 
As análises e as discussões dos resultados obtidos no presente trabalho permitiram as seguintes conclusões:

1 A cirurgia de correção da $\mathrm{IM}$ determinou uma diminuição significativa do $\mathrm{VO}_{2}$ pico e do OUES, no TCPE realizado na bicicleta.

2 A porcentagem dos valores preditos do $\mathrm{VO}_{2}$ pico e do OUES se mantiveram, em média, abaixo de $70 \%$ desde o pré-operatório no TCPE bicicleta.

3 No TCPE bicicleta, o pulso de $\mathrm{O}_{2}$ aumentou nos pacientes submetidos ao reparo mitral.

4 Houve correlação negativa das diferenças relativas do pulso de $\mathrm{O}_{2}$ e do OUES com as de FE e PSAP, respectivamente, no TCPE bicicleta.

5 A variável $\mathrm{VE} / \mathrm{VCO}_{2}$ slope não apresentou diferença significativa após a cirurgia no TCPE bicicleta, assim como não houve correlação com as medidas ecocardiográficas de remodelamento cardíaco.

6 No TCPE realizado na esteira, houve leve diminuição não significativa do $\mathrm{VO}_{2}$ pico após a cirurgia, porém a diferença relativa do $\mathrm{VO}_{2}$ apresentou correlação negativa com a da PSAP.

7 A variável OUES apresentou redução pós-operatória no TCPE na esteira e esta redução se correlacionou, inversamente, com a diferença relativa de VDFVE, VAE e de PSAP. 
$8 \mathrm{O} \mathrm{VO}_{2}$ pico e o OUES após a cirurgia, avaliado pelo TCPE na bicicleta, foram maiores no grupo reparo mitral em relação a troca valvar.

9 Após a cirurgia, a redução significativa do $\mathrm{VO}_{2}$ pico avaliado pelo TCPE na bicicleta foi observada apenas nos pacientes do grupo troca valvar mitral.

10 Não houve diferença significativa do OUES nos grupos troca valvar mitral e reparo mitral, no TCPE realizado na esteira. 
Anexos 
ANEXO A Formulário de consentimento do paciente ou responsável

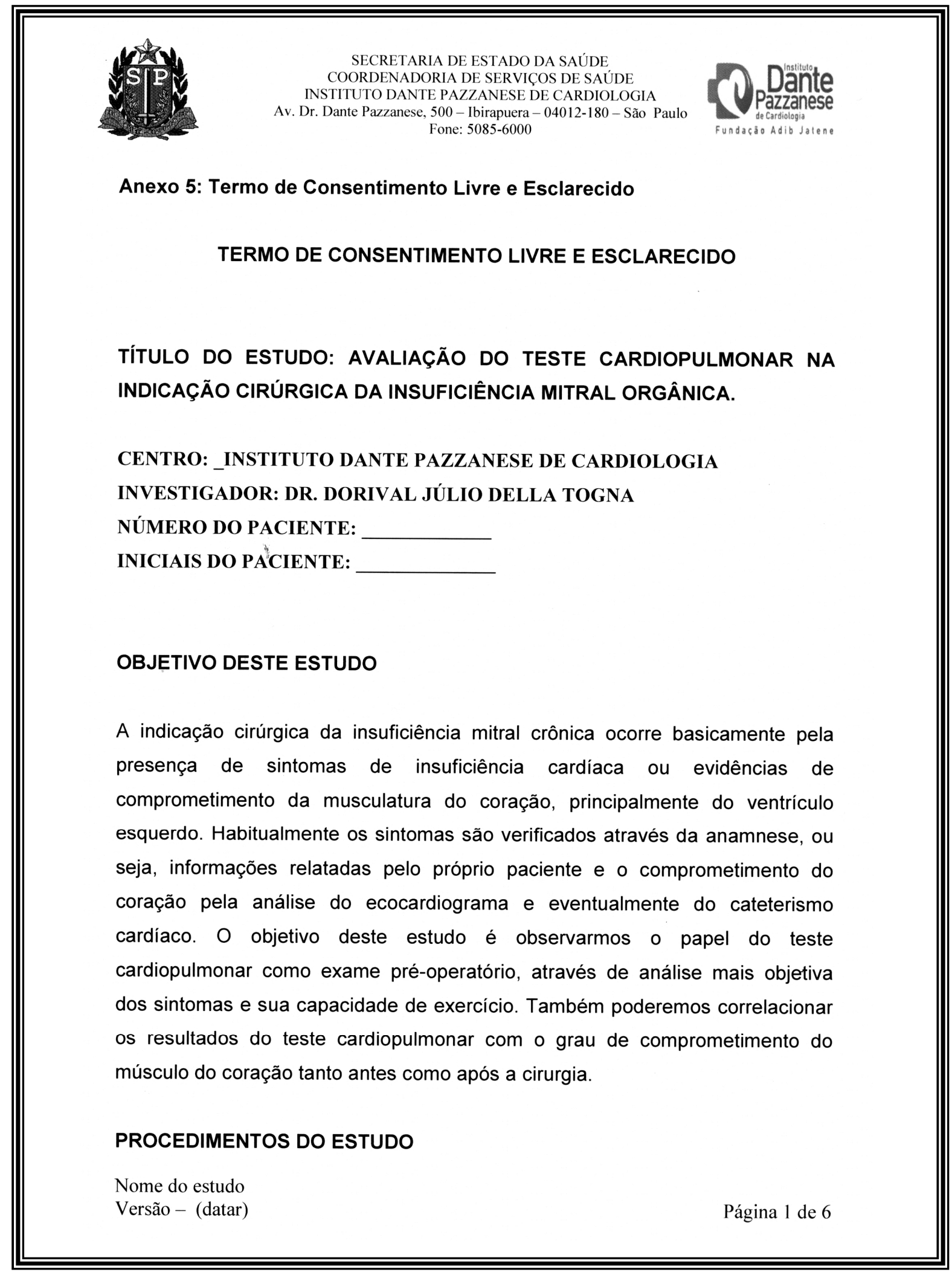


ANEXO A continuação

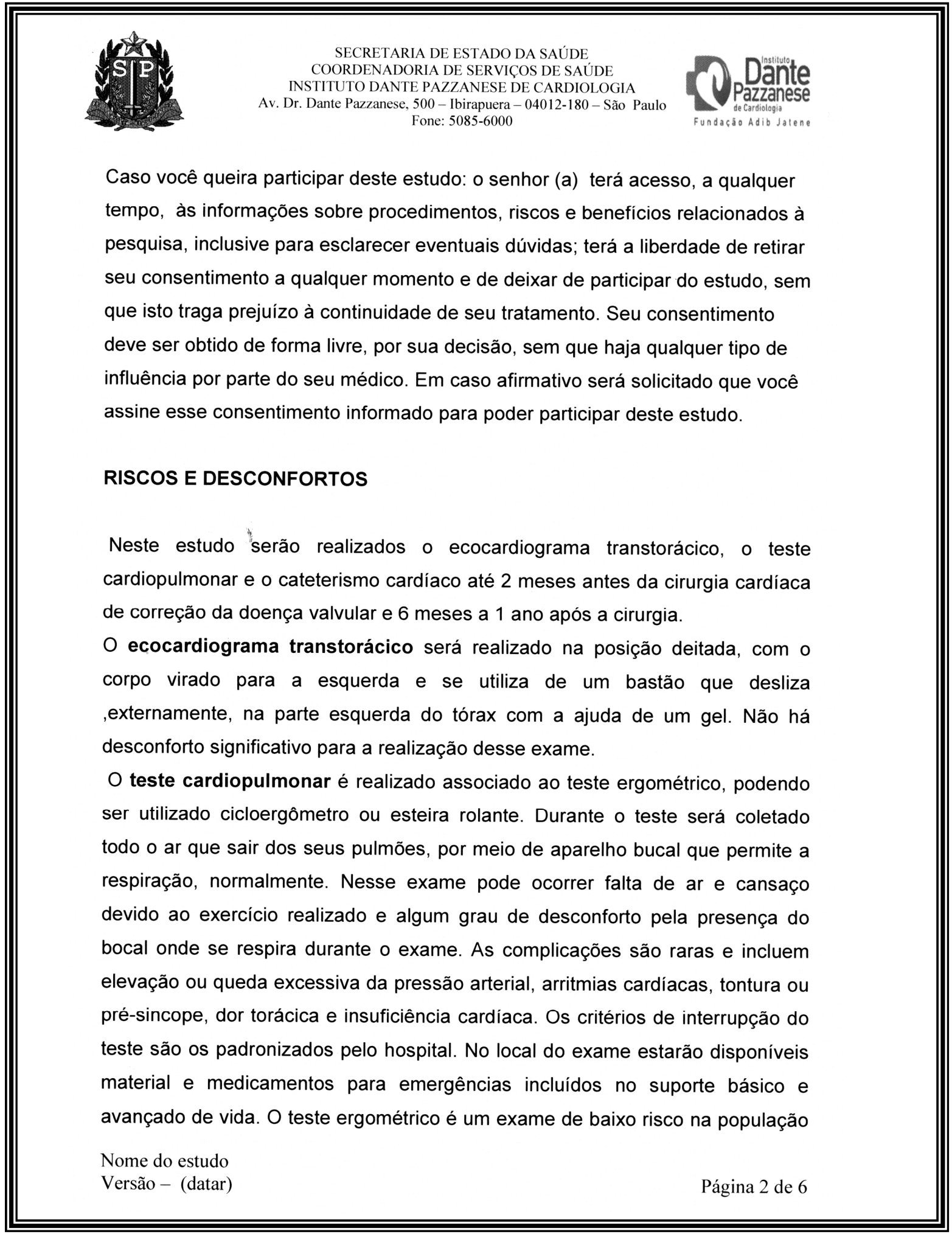

continua 
ANEXO A continuação

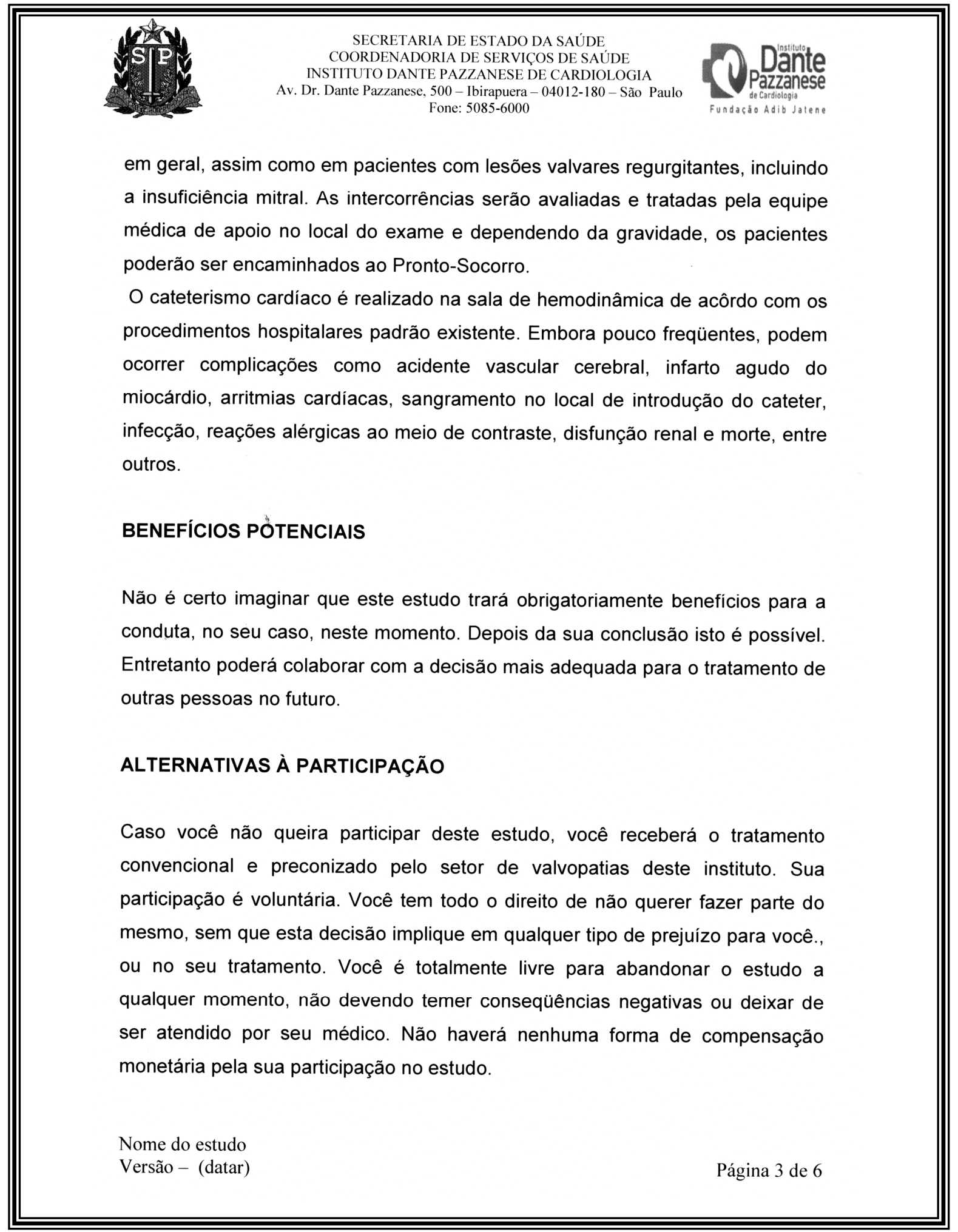

continua 
ANEXO A continuação

\begin{tabular}{|c|}
\hline 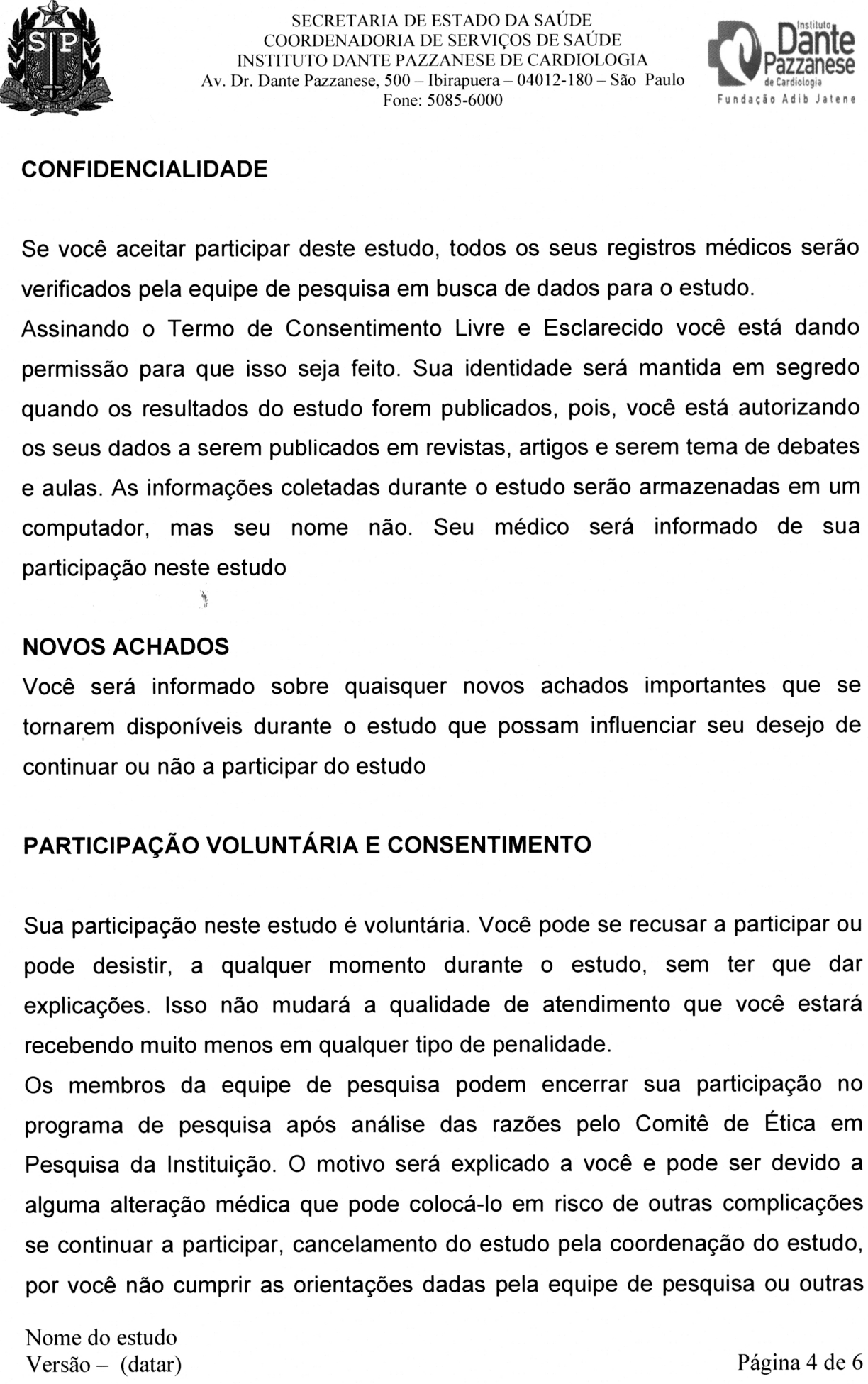 \\
\hline
\end{tabular}


ANEXO A continuação

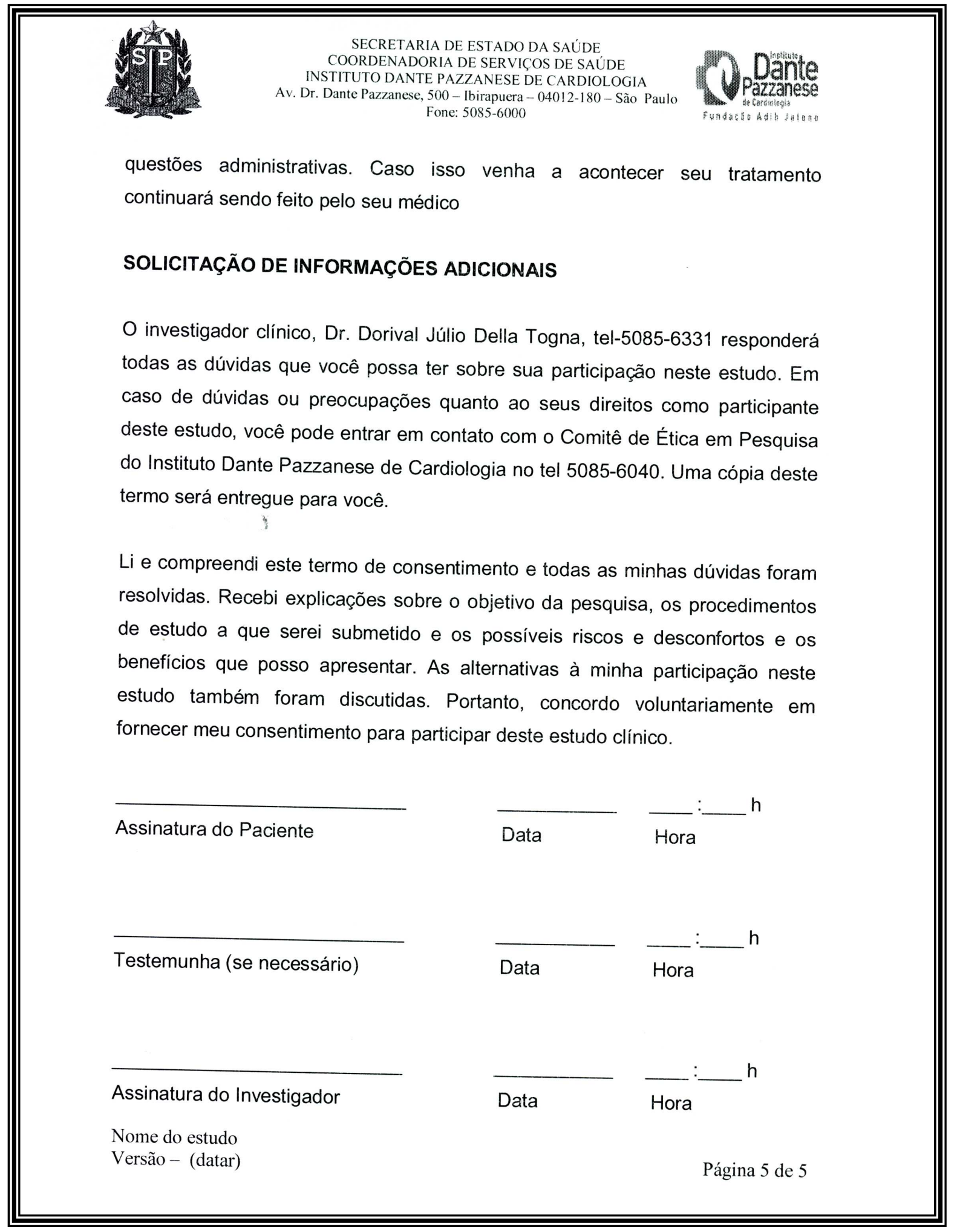


Referências Bibliográficas 
1 Singh JP, Evans JC, Levy D, Larson MG, Freed LA, et al. Prevalence and clinical determinants of mitral, tricuspid, and aortic regurgitation (the Framingham Heart Study). Am J Cardiol 1999;83(6):897-902.

2 Nkomo VT, Gardin JM, Skelton TN, Gottdiener JS, Scott CG, EnriquezSarano M. Burden of valvular heart diseases: a population-based study. Lancet 2006;368(9540):1005-11.

3 lung B, Baron G, Butchart EG, Delahaye F, Gohlke-Barwolf C, et al. A prospective survey of patients with valvular heart disease in Europe: The Euro Heart Survey on Valvular Heart Disease. Eur Heart $J$ $2003 ; 24(13): 1231-43$.

4 Essop MR, Nkomo VT. Rheumatic and nonrheumatic valvular heart disease: epidemiology, management, and prevention in Africa. Circulation 2005;112(23):3584-9.

5 Kaplan EL. Recent epidemiology of group A streptococcal infections in North America and abroad: an overview. Pediatrics 1996;97(6 Pt 2):945-8.

6 Rheumatic fever and rheumatic heart disease: report of a WHO expert consultation on rheumatic fever and rheumatic heart disease. World Health Organization. Geneva, 2001 Oct 29 - Nov 1. Geneva: WHO; 2004. 
7 da Silva NA, Pereira BA. Acute rheumatic fever. Still a challenge. Rheum Dis Clin North Am 1997;23(3):545-68.

8 Fonte: Ministério da Saúde - Sistema de Informações Hospitalares do SUS (SIH/SUS) 2011. In: http://www.datasus.gov.br/tabnet/tabnet.htm.

9 Otto CM, Salerno CT. Timing of surgery in asymptomatic mitral regurgitation. N Engl J Med 2005;352(9):928-9.

10 Kang DH, Kim JH, Rim JH, Kim MJ, Yun SC, Song JM, et al. Comparison of early surgery versus conventional treatment in asymptomatic severe mitral regurgitation. Circulation 2009;119(6):797804.

11 Rosenhek R, Rader F, Klaar U, Gabriel H, Krejc M, Kalbeck D, et al. Outcome of watchful waiting in asymptomatic severe mitral regurgitation. Circulation 2006;113(18):2238-44.

12 Griffin BP. Timing of surgical intervention in chronic mitral regurgitation: is vigilance enough? Circulation 2006 9;113(18):2169-72.

13 Ngaage DL, Schaff HV. Mitral valve surgery in non-ischemic cardiomyopathy. J Cardiovasc Surg 2004;45(5):477-86.

14 Zoghbi WA, Enriquez-Sarano M, Foster E, Grayburn PA, Kraft CD, Levine RA, et al. Recommendations for evaluation of the severity of native valvular regurgitation with two-dimensional and Doppler echocardiography. J Am Soc Echocardiogr 2003;16(7):777-802.

15 Enriquez-Sarano M, Basmadjian AJ, Rossi A, Bailey KR, Seward JB, Tajik AJ. Progression of mitral regurgitation: a prospective Doppler echocardiographic study. J Am Coll Cardiol 1999;34(4):1137-4. 
16 Eckberg DL, Gault JH, Bouchard RL, Karliner JS, Ross J, Jr. Mechanics of left ventricular contraction in chronic severe mitral regurgitation. Circulation 1973;47(6):1252-9.

17 Ross J, Jr. Left ventricular function and the timing of surgical treatment in valvular heart disease. Ann Intern Med 1981;94(4 pt 1):498-504.

18 Delahaye JP, Gare JP, Viguier E, Delahaye F, De GG, Milon H. Natural history of severe mitral regurgitation. Eur Heart J 1991;12 Suppl B:5-9.

19 Carabello BA. The pathophysiology of mitral regurgitation. J Heart Valve Dis 2000;9(5):600-8.

20 Schuler G, Peterson KL, Johnson A, Francis G, Dennish G, Utley J, et al. Temporal response of left ventricular performance to mitral valve surgery. Circulation 1979;59(6):1218-31.

21 Carabello BA, Nolan SP, McGuire LB. Assessment of preoperative left ventricular function in patients with mitral regurgitation: value of the endsystolic wall stress-end-systolic volume ratio. Circulation $1981 ; 64(6): 1212-7$

22 Timmis SB, Kirsh MM, Montgomery DG, Starling MR. Evaluation of left ventricular ejection fraction as a measure of pump performance in patients with chronic mitral regurgitation. Catheter Cardiovasc Interv 2000;49(3):290-6.

23 Enriquez-Sarano M, Tajik AJ, Schaff HV, Orszulak TA, McGoon MD, Bailey KR, et al. Echocardiographic prediction of left ventricular function after correction of mitral regurgitation: results and clinical implications. $J$ Am Coll Cardiol 1994;24(6):1536-43. 
24 Crawford $\mathrm{MH}$, Souchek J, Oprian CA, Miller DC, Rahimtoola S, Giacomini JC, et al. Determinants of survival and left ventricular performance after mitral valve replacement. Department of Veterans Affairs Cooperative Study on Valvular Heart Disease. Circulation 1990;81(4):1173-81.

25 Carabello BA, Nolan SP, McGuire LB. Assessment of preoperative left ventricular function in patients with mitral regurgitation: value of the endsystolic wall stress-end-systolic volume ratio. Circulation $1981 ; 64(6): 1212-7$.

26 Braunberger E, Deloche A, Berrebi A, Abdallah F, Celestin JA, Meimoun $\mathrm{P}$, et al. Very long-term results (more than 20 years) of valve repair with carpentier's techniques in nonrheumatic mitral valve insufficiency. Circulation 2001;104(12 Suppl 1):I8-11.

27 Flameng W, Herijgers P, Bogaerts K. Recurrence of mitral valve regurgitation after mitral valve repair in degenerative valve disease. Circulation 2003;107(12):1609-13.

28 Jokinen JJ, Hippelainen MJ, Pitkanen OA, Hartikainen JE. Mitral valve replacement versus repair: propensity-adjusted survival and quality-oflife analysis. Ann Thorac Surg 2007;84(2):451-8.

29 Shuhaiber J, Anderson RJ. Meta-analysis of clinical outcomes following surgical mitral valve repair or replacement. Eur $J$ Cardiothorac Surg $2007 ; 31(2): 267-75$. 
30 Enriquez-Sarano M, Schaff HV, Orszulak TA, Tajik AJ, Bailey KR, Frye RL. Valve repair improves the outcome of surgery for mitral regurgitation. A multivariate analysis. Circulation 1995;91(4):1022-8.

31 Corin WJ, Sutsch G, Murakami T, Krogmann ON, Turina M, Hess OM. Left ventricular function in chronic mitral regurgitation: preoperative and postoperative comparison. J Am Coll Cardiol 1995;25(1):113-21.

32 Rozich JD, Carabello BA, Usher BW, Kratz JM, Bell AE, Zile MR. Mitral valve replacement with and without chordal preservation in patients with chronic mitral regurgitation. Mechanisms for differences in postoperative ejection performance. Circulation 1992;86(6):1718-26.

33 Goldman ME, Mora F, Guarino T, Fuster V, Mindich BP. Mitral valvuloplasty is superior to valve replacement for preservation of left ventricular function: an intraoperative two-dimensional echocardiographic study. J Am Coll Cardiol 1987;10(3):568-75.

34 Tischler MD, Cooper KA, Rowen M, LeWinter MM. Mitral valve replacement versus mitral valve repair. A Doppler and quantitative stress echocardiographic study. Circulation 1994;89(1):132-7.

35 Natsuaki M, Itoh T, Tomita S, Furukawa K, Yoshikai M, Suda H, et al. Importance of preserving the mitral subvalvular apparatus in mitral valve replacement. Ann Thorac Surg 1996;61(2):585-90.

36 Lee EM, Shapiro LM, Wells FC. Importance of subvalvular preservation and early operation in mitral valve surgery. Circulation 1996;94(9):211723. 
37 Le TT, de GP, Millaire A, Foucher C, Savoye C, Pigny P, et al. Effect of mitral valve surgery on exercise capacity, ventricular ejection fraction and neurohormonal activation in patients with severe mitral regurgitation. J Am Coll Cardiol 2000;36(7):2263-9.

38 Sousa UM, Dreyfus G, Rescigno G, al AN, Mascagni R, La MM, et al. Surgical treatment of asymptomatic and mildly symptomatic mitral regurgitation. J Thorac Cardiovasc Surg 1996;112(5):1240-8.

39 Barnett SD, Ad N. Surgery for aortic and mitral valve disease in the United States: a trend of change in surgical practice between 1998 and 2005. J Thorac Cardiovasc Surg 2009;137(6):1422-9.

40 Gammie JS, Sheng S, Griffith BP, Peterson ED, Rankin JS, O'Brien $\mathrm{SM}$, et al. Trends in mitral valve surgery in the United States: results from the Society of Thoracic Surgeons Adult Cardiac Surgery Database. Ann Thorac Surg 2009;87(5):1431-7.

41 Enriquez-Sarano M, Tajik AJ, Schaff HV, Orszulak TA, Bailey KR, Frye RL. Echocardiographic prediction of survival after surgical correction of organic mitral regurgitation. Circulation 1994;90(2):830-7.

42 Tribouilloy CM, Enriquez-Sarano M, Schaff HV, Orszulak TA, Bailey $\mathrm{KR}$, Tajik AJ, et al. Impact of preoperative symptoms on survival after surgical correction of organic mitral regurgitation: rationale for optimizing surgical indications. Circulation 1999;99(3):400-5.

43 Enriquez-Sarano M, Avierinos JF, Messika-Zeitoun D, Detaint D, Capps M, Nkomo V, et al. Quantitative determinants of the outcome of asymptomatic mitral regurgitation. N Engl J Med 2005;352(9):875-83. 
44 Yamano T, Gillinov AM, Wada N, Matsumura Y, Toyono M, Thomas JD, et al. Doppler-derived preoperative mitral regurgitation volume predicts postoperative left ventricular dysfunction after mitral valve repair. Am Heart J 2009;157(5):875-82.

45 Bonow RO, Carabello BA, Kanu C, de Leon ACJ, Faxon DP, Freed MD, et al. ACC/AHA 2006 guidelines for the management of patients with valvular heart disease: a report of the American College of Cardiology/American Heart Association Task Force on Practice Guidelines (writing committee to revise the 1998 Guidelines for the Management of Patients With Valvular Heart Disease): developed in collaboration with the Society of Cardiovascular Anesthesiologists: endorsed by the Society for Cardiovascular Angiography and Interventions and the Society of Thoracic Surgeons. Circulation 2006;114(5):e84-231.

46 Ling LH, Enriquez-Sarano M, Seward JB, Tajik AJ, Schaff HV, Bailey $\mathrm{KR}$, et al. Clinical outcome of mitral regurgitation due to flail leaflet. $N$ Engl J Med 1996;335(19):1417-23.

47 Vahanian A, Baumgartner H, Bax J, Butchart E, Dion R, Filippatos G, et al. Guidelines on the management of valvular heart disease: The Task Force on the Management of Valvular Heart Disease of the European Society of Cardiology. Eur Heart J 2007;28(2):230-68.

48 Quinones MA. Management of mitral regurgitation. Optimal timing for surgery. Cardiol Clin 1998;16(3):421-35, viii. 
49 Scano G, Ambrosino N. Pathophysiology of dyspnea. Lung $2002 ; 180(3): 131-48$.

50 The Criteria Committee of the New York Heart Association: Nomenclature and Criteria for Diagnosis of Diseases of the Heart and Great Vessels. 9 ${ }^{\text {th }}$ Ed. Boston, Little, Brown, 1994.

51 Bettencourt P, Ferreira A, Dias P, Pimenta J, Frioes F, Martins L, et al. Predictors of prognosis in patients with stable mild to moderate heart failure. J Card Fail 2000;6(4):306-13.

52 [III Guidelines of Sociedade Brasileira de Cardiologia on the exercise test]. Arq Bras Cardiol 2010;95(5 Suppl 1):1-26.

53 Gohlke-Barwolf C, Gohlke H, Samek L, Peters K, Betz P, Eschenbruch E, et al. Exercise tolerance and working capacity after valve replacement. J Heart Valve Dis 1992;1(2):189-95.

54 Weber KT, Kinasewitz GT, Janicki JS, Fishman AP. Oxygen utilization and ventilation during exercise in patients with chronic cardiac failure. Circulation 1982 Jun;65(6):1213-23.

55 Palange $\mathrm{P}$, Ward SA, Carlsen KH, Casaburi R, Gallagher CG, Gosselink R, et al. Recommendations on the use of exercise testing in clinical practice. Eur Respir J 2007;29(1):185-209.

56 Arena R, Myers J, Williams MA, Gulati M, Kligfield P, Balady GJ, et al. Assessment of functional capacity in clinical and research settings: a scientific statement from the American Heart Association Committee on Exercise, Rehabilitation, and Prevention of the Council on Clinical 
Cardiology and the Council on Cardiovascular Nursing. Circulation 2007;116(3):329-43.

57 Bissessor N, Stewart R, Wee YS, Zeng I, Jayasinghe R, Howes L, et al. Complex valve disease: pre-surgical functional capacity evaluation using peak oxygen consumption. J Heart Valve Dis 2009;18(5):554-61.

58 Kim HJ, Ahn SJ, Park SW, Cho BR, Sung J, Hong SH, et al. Cardiopulmonary exercise testing before and one year after mitral valve repair for severe mitral regurgitation. Am J Cardiol 2004;93(9):1187-9.

59 Horstkotte D, Niehues R, Schulte HD, Strauer BE. [Exercise capacity after heart valve replacement]. Z Kardiol 1994;83 Suppl 3:111-20.

60 Tamai J, Kosakai Y, Yoshioka T, Ohnishi E, Takaki H, Okano Y, et al. Delayed improvement in exercise capacity with restoration of sinoatrial node response in patients after combined treatment with surgical repair for organic heart disease and the Maze procedure for atrial fibrillation. Circulation 1995;91(9):2392-9.

61 Nakamura M, Chiba M, Ueshima K, Arakawa N, Yoshida H, Makita S, et al. Effects of mitral and/or aortic valve replacement or repair on endothelium-dependent peripheral vasorelaxation and its relation to improvement in exercise capacity. Am J Cardiol 1996;77(1):98-102.

62 ul Haque ME, Sasaki S, Kuroda H, Ishiguro S, Ogino K, Kobayashi T, et al. Hemodynamic changes during dynamic exercise in patients after mitral valve replacement for chronic mitral regurgitation. Indian Heart $J$ 1992;44(6):379-85. 
63 [Directory for interpretation of electrocardiograms at rest]. Arq Bras Cardiol 2003;80 Suppl 2:1-17.

64 Lang RM, Bierig M, Devereux RB, Flachskampf FA, Foster E, Pellikka PA, et al. Recommendations for chamber quantification: a report from the American Society of Echocardiography's Guidelines and Standards Committee and the Chamber Quantification Writing Group, developed in conjunction with the European Association of Echocardiography, a branch of the European Society of Cardiology. J Am Soc Echocardiogr 2005;18(12):1440-63.

65 Quinones MA, Otto CM, Stoddard M, Waggoner A, Zoghbi WA. Recommendations for quantification of Doppler echocardiography: a report from the Doppler Quantification Task Force of the Nomenclature and Standards Committee of the American Society of Echocardiography. J Am Soc Echocardiogr 2002;15(2):167-84.

66 Diretrizes para testes de função pulmonar. J Bras Pneumol 2002; 28(supl.3):S2-S162.

67 Porszasz J, Barstow TJ, Wasserman K. Evaluation of a symmetrically disposed Pitot tube flowmeter for measuring gas flow during exercise. $J$ Appl Physiol 1994;77(6):2659-65.

68 Borg GA. Psychophysical bases of perceived exertion. Med Sci Sports Exerc 1982;14(5):377-81.

69 Baim DS. Grossman's Cardiac Catheterization, Angiography, and Intervention. Seventh Edition. Lippincott Williams \& Wilkins. 2006. Section II: Basic Tecniques .p.79-106. 
70 Siegel S, Castellan NJ. Nonparametric Statistics. New York: McGrawHill. 2ed. 1988.

71 Brunner E, Langer F. Nonparametric analysis of ordered categorical data in designs with longitudinal observations and small sample sizes. Biometrical Journal 2000, 42, 663-675.

72 Pereira JCR. Bioestatística em Outras Palavras. 1 ed. EDUSP-SP, 2010.

73 A Armitage P, Berry G. Statistical Methods in Medical Research. 3 ed. Oxford: Blackwell Science, 1994.

74 Neter J, Kutner MH, Nachtsheim CJ, Wasserman W. Applied Linear Statistical Models. $4^{\underline{a}}$ ed. U.S.A.: Times Mirror Higher Education Group.), 1996.

75 Mezzani A, Agostoni P, Cohen-Solal A, Corra U, Jegier A, Kouidi E, et al. Standards for the use of cardiopulmonary exercise testing for the functional evaluation of cardiac patients: a report from the Exercise Physiology Section of the European Association for Cardiovascular Prevention and Rehabilitation. Eur J Cardiovasc Prev Rehabil 2009;16(3):249-679 .

76 Sellier P, Chatellier G, D'Agrosa-Boiteux MC, Douard H, Dubois C, Goepfert PC, et al. Use of non-invasive cardiac investigations to predict clinical endpoints after coronary bypass graft surgery in coronary artery disease patients: results from the prognosis and evaluation of risk in the coronary operated patient (PERISCOP) study. Eur Heart $J$ 2003;24(10):916-26. 
77 Weissman C. Pulmonary function after cardiac and thoracic surgery. Anesth Analg 1999;88(6):1272-9.

78 Raphael C, Briscoe C, Davies J, lan WZ, Manisty C, Sutton R, et al. Limitations of the New York Heart Association functional classification system and self-reported walking distances in chronic heart failure. Heart 2007;93(4):476-82.

79 Balady GJ, Arena R, Sietsema K, Myers J, Coke L, Fletcher GF, et al. Clinician's Guide to cardiopulmonary exercise testing in adults: a scientific statement from the American Heart Association. Circulation 2010;122(2):191-225.

80 Messika-Zeitoun D, Johnson BD, Nkomo V, Avierinos JF, Allison TG, Scott C, et al. Cardiopulmonary exercise testing determination of functional capacity in mitral regurgitation: physiologic and outcome implications. J Am Coll Cardiol 2006;47(12):2521-7.

81 Neder J.A.; Nery, L.E. Teste de Exercício Cardiopulmonar. Jornal de Pneumologia, v. 28, supl. 3, 2002.

82 Reed D, Abbott RD, Smucker ML, Kaul S. Prediction of outcome after mitral valve replacement in patients with symptomatic chronic mitral regurgitation. The importance of left atrial size. Circulation 1991;84(1):23-34.

83 Piepoli MF, Corra U, Agostoni PG, Belardinelli R, Cohen-Solal A, Hambrecht $\mathrm{R}$, et al. Statement on cardiopulmonary exercise testing in chronic heart failure due to left ventricular dysfunction: recommendations for performance and interpretation. Part I: definition 
of cardiopulmonary exercise testing parameters for appropriate use in chronic heart failure. Eur J Cardiovasc Prev Rehabil 2006;13(2):150-64.

84 Oliveira RB, Myers J, Araujo CG, Abella J, Mandic S, Froelicher V. Maximal exercise oxygen pulse as a predictor of mortality among male veterans referred for exercise testing. Eur J Cardiovasc Prev Rehabil 2009;16(3):358-64.

85 Cohen-Solal A, Tabet JY, Logeart D, Bourgoin P, Tokmakova M, Dahan M. A non-invasively determined surrogate of cardiac power ('circulatory power') at peak exercise is a powerful prognostic factor in chronic heart failure. Eur Heart J 2002;23(10):806-14.

86 Arena R, Myers J, Abella J, Peberdy MA, Bensimhon D, Chase P, et al. The ventilatory classification system effectively predicts hospitalization in patients with heart failure. J Cardiopulm Rehabil Prev 2008;28(3):195-8.

87 Defoor J, Schepers D, Reybrouck T, Fagard R, Vanhees L. Oxygen uptake efficiency slope in coronary artery disease: clinical use and response to training. Int J Sports Med 2006;27(9):730-7.

88 Van LC, Van D, V, De BG, Bihija S, Seghers T, Cambier D, et al. Response of the oxygen uptake efficiency slope to exercise training in patients with chronic heart failure. Eur J Heart Fail 2007;9(6-7):625-9.

89 Hollenberg M, Tager IB. Oxygen uptake efficiency slope: an index of exercise performance and cardiopulmonary reserve requiring only submaximal exercise. J Am Coll Cardiol 2000;36(1):194-201. 
90 Pogliaghi S, Dussin E, Tarperi C, Cevese A, Schena F. Calculation of oxygen uptake efficiency slope based on heart rate reserve end-points in healthy elderly subjects. Eur J Appl Physiol 2007;101(6):691-6.

91 Arena R, Lavie CJ, Milani RV, Myers J, Guazzi M. Cardiopulmonary exercise testing in patients with pulmonary arterial hypertension: an evidence-based review. J Heart Lung Transplant 2010;29(2):159-73.

92 Hambrecht R, Walther C, Mobius-Winkler S, Gielen S, Linke A, Conradi $\mathrm{K}$, et al. Percutaneous coronary angioplasty compared with exercise training in patients with stable coronary artery disease: a randomized trial. Circulation 2004;109(11):1371-8.

93 Belardinelli R, Georgiou D, Cianci G, Purcaro A. Randomized, controlled trial of long-term moderate exercise training in chronic heart failure: effects on functional capacity, quality of life, and clinical outcome. Circulation 1999;99(9):1173-82.

94 McKelvie RS, Teo KK, Roberts R, McCartney N, Humen D, Montague $\mathrm{T}$, et al. Effects of exercise training in patients with heart failure: the Exercise Rehabilitation Trial (EXERT). Am Heart J 2002;144(1):23-30.

95 Giannuzzi P, Temporelli PL, Corra U, Tavazzi L. Antiremodeling effect of long-term exercise training in patients with stable chronic heart failure: results of the Exercise in Left Ventricular Dysfunction and Chronic Heart Failure (ELVD-CHF) Trial. Circulation 2003;108(5):554-9.

96 Vanhees L, Fagard R, Thijs L, Amery A. Prognostic value of traininginduced change in peak exercise capacity in patients with myocardial 
infarcts and patients with coronary bypass surgery. Am $J$ Cardiol 1995;76(14):1014-9.

97 Nakai Y, Kataoka Y, Bando M, Hiasa Y, Taki H, Harada M, et al. Effects of physical exercise training on cardiac function and graft patency after coronary artery bypass grafting. I Thorac Cardiovasc Surg 1987;93(1):65-72.

98 Hedback BE, Perk J, Engvall J, Areskog NH. Cardiac rehabilitation after coronary artery bypass grafting: effects on exercise performance and risk factors. Arch Phys Med Rehabil 1990;71(13):1069-73.

99 Gohlke-Barwolf C, Gohlke H, Samek L, Peters K, Betz P, Eschenbruch $\mathrm{E}$, et al. Exercise tolerance and working capacity after valve replacement. J Heart Valve Dis 1992;1(2):189-95.

100 Meurin P, lliou MC, Ben DA, Pierre B, Corone S, Cristofini P, et al. Early exercise training after mitral valve repair: a multicentric prospective French study. Chest 2005;128(3):1638-44. 